\title{
Radiative Torques on Interstellar Grains. III. Dynamics with Thermal Relaxation
}

\author{
Joseph C. Weingartner \\ CITA, 60 St. George Street, University of Toronto, Toronto, ON M5S 3H8, Canada; \\ weingart@cita.utoronto.ca \\ and \\ B.T. Draine \\ Princeton University Observatory, Peyton Hall, Princeton, NJ 08544, USA; \\ draine@astro.princeton.edu
}

\begin{abstract}
In the previous papers in this series, we found that radiative torques can play a major role in the alignment of grains with the interstellar magnetic field. Since the radiative torques can drive the grains to suprathermal rotational speeds, in previous work we made the simplifying assumption that the grain principal axis of greatest moment of inertia is always parallel to the grain angular momentum. This enabled us to describe many of the features of the grain dynamics. However, this assumption fails when the grains enter periods of thermal rotation, which occur naturally in the radiative torque alignment scenario. In the present paper, we relax this assumption and explore the consequences for the grain dynamics. We develop a treatment to follow the grain dynamics including thermal fluctuations and "thermal flipping", and show results for one illustrative example. By comparing with a treatment without thermal fluctuations, we see that inclusion of thermal fluctuations can lead to qualitative changes in the grain dynamics. In a future installment in this series, we will use the more complete dynamical treatment developed here to perform a systematic study of grain alignment by radiative torques.
\end{abstract}

Subject headings: ISM: dust, extinction — polarization — scattering

\section{Introduction}

Polarization of starlight by the interstellar medium was discovered serendipitously (Hall 1949; Hall \& Mikesell 1949; Hiltner 1949a, b). It was immediately recognized that the polarization must be due to selective extinction by dust grains, and that this required that interstellar dust grains be both nonspherical and aligned. The first papers seeking to account for the observed 
alignment appeared almost immediately (Spitzer \& Schatzman 1949; Spitzer \& Tukey 1949; Davis \& Greenstein 1951; Spitzer \& Tukey 1951), but a satisfactory explanation for the grain alignment has eluded theoretical understanding for many decades, despite numerous investigations (see Lazarian 2002 for a recent review).

Davis \& Greenstein (1951) suggested that the alignment results from paramagnetic dissipation. For this mechanism to act, the grain must be paramagnetic and it must rotate through the static interstellar magnetic field. Paramagnetism appears likely for amorphous silicate grains, and plausible for hydrogenated carbonaceous grains. Indeed, given the substantial fraction of the overall grain mass contributed by Fe, a significant ferromagnetic or ferrimagnetic fraction would not be impossible.

Davis \& Greenstein assumed that the grain rotation is excited by elastic impacts with gas atoms. In this case, the energy in rotation about any grain axis is $\sim \frac{1}{2} k T_{\text {gas }}$, where $k$ is Boltzmann's constant and $T_{\text {gas }}$ is the gas temperature; thus, this motion is called "thermal rotation". The grain rotating through a static magnetic field is analogous to a magnetic resonance experiment, in which a static solid is exposed to a rotating field. In both cases, the material tries to magnetize along the field direction, but with a lag, and energy is dissipated into heat. In the magnetic resonance experiment, the dissipated energy originates in the radiation field, whereas in the interstellar case, the grain's rotational energy is dissipated.

The statistical mechanics of a rigid paramagnetic grain was analyzed by Jones \& Spitzer (1967) and Purcell \& Spitzer (1971). If the gas temperature $T_{\text {gas }}$ exceeds the dust temperature $T_{d}$ and if there were no disalignment due to random collisions with gas atoms, then the Davis-Greenstein mechanism would drive a grain to tend to spin about its principal axis of greatest moment of inertia (hereafter $\hat{\mathbf{a}}_{1}$ ), which would tend to be aligned with the local interstellar magnetic field $\mathbf{B}$.

However, for typical conditions in the diffuse ISM, the timescale for disalignment (due to collisions with gas atoms) is substantially shorter than the Davis-Greenstein alignment timescale $\tau_{\text {DG }}$. Jones \& Spitzer (1967) showed that if grains have superparamagnetic inclusions (e.g., domains of pure Fe) then $\tau_{\mathrm{DG}}$ could be reduced by several orders of magnitude. Mathis (1986) noted that, since larger grains would be more likely to contain one or more such inclusions, this could explain the observation that relatively large grains $(a \gtrsim 0.1 \mu \mathrm{m})$ are well aligned while smaller grains $(a \lesssim 0.05 \mu \mathrm{m})$ are not (see, e.g., Kim \& Martin 1995). ${ }^{1}$

Research on the rotational dynamics of interstellar grains discovered important effects which had hitherto been overlooked. Martin (1971) pointed out that a charged spinning grain has a magnetic moment parallel or anti-parallel to the angular velocity $\boldsymbol{\omega}$, so that the grain precesses about $\mathbf{B}$. The precession rate is fast enough that, even if the actual grain alignment mechanism did not involve $\mathbf{B}$, the observed polarization would be either parallel or perpendicular to $\mathbf{B}$. Dolginov \& Mytrophanov (1976) showed that the Barnett effect (the tendency for a spinning body to become

\footnotetext{
${ }^{1}$ Throughout this paper, we characterize the grain size by the radius $a$ of a sphere of equal volume.
} 
magnetized antiparallel to $\boldsymbol{\omega})$ provides a much larger magnetic moment.

The problem of grain alignment therefore cleanly separates into two issues: (1) the alignment of grain angular momentum $\mathbf{J}$ with $\mathbf{B}$, and (2) the alignment of the grain itself with its instantaneous angular momentum $\mathbf{J}$.

Purcell (1979) realized that the Barnett effect would lead rapidly-rotating grains to tend to spin about $\hat{\mathbf{a}}_{1}$, its principal axis of largest moment of inertia. This is the configuration for which a grain with constant angular momentum $\mathbf{J}$ has minimum rotational kinetic energy. If the grain starts in a different configuration, then generally the magnetization due to the Barnett effect changes direction (in a periodic manner) in grain body coordinates; the resulting paramagnetic dissipation (which Purcell called "Barnett dissipation" in this case) drives the grain to its state with minimum rotational energy. ${ }^{2}$ Purcell found that the alignment of $\hat{\mathbf{a}}_{1}$ with $\mathbf{J}$ occurs on a much shorter timescale than $\tau_{\mathrm{DG}}$ or the gas-drag timescale, if the grain rotational kinetic energy $\gg k T_{d}$. The Barnett dissipation is necessarily accompanied by thermal fluctuations which act to disalign $\hat{\mathbf{a}}_{1}$ from $\mathbf{J}$ (Lazarian \& Roberge 1997). Roberge \& Lazarian (1999) have calculated the Davis-Greenstein alignment of oblate spheroidal grains with this effect included.

Purcell $(1975,1979)$ also pointed out that grains are subject to systematic torques fixed in grain body coordinates, and that these torques can spin the grain up to suprathermal rotational speeds. A suprathermally-rotating grain is largely impervious to disalignment by random gas atom bombardment, and is thus free to undergo alignment on the Davis-Greenstein timescale. However, the systematic torques discussed by Purcell result from processes that occur at the grain surface (e.g., the formation of $\mathrm{H}_{2}$ at special surface sites, with subsequent ejection), and thus depend sensitively on the details of the grain surface. Changes in the surface (e.g., due to accretion of atoms from the gas) can change the magnitude and direction of the systematic torque. As a result, the grain will sometimes be spun down, when the torque is directed opposite $\boldsymbol{\omega}$, and the grain rotational kinetic energy can be reduced to $\sim k T_{\text {gas }}$ for a period of time. Such episodes are called "crossovers". 3

Crossovers were first studied by Spitzer \& McGlynn (1979). They found that, in the absence of stochastic torques, the direction of $\mathbf{J}$ remains constant. The magnitude of $\mathbf{J}$ decreases and then increases again; during this episode the grain flips over. With stochastic torques, the scenario is largely the same, except that the direction of $\mathbf{J}$ after the crossover is not identical to its initial direction. Spitzer \& McGlynn concluded that a grain would become completely disaligned after passing through a small number of crossovers. This implies that if crossovers occur frequently (on a timscale shorter than $\tau_{\mathrm{DG}}$ ), then paramagnetic relaxation (without superparamagnetic inclusions)

\footnotetext{
${ }^{2}$ Even in the absence of Barnett dissipation, viscoelastic dissipation would align $\hat{\mathbf{a}}_{1}$ with $\mathbf{J}$; Purcell found that Barnett dissipation is stronger.

${ }^{3}$ The systematic torque need not be directed exactly antiparallel to $\mathbf{J}$ to result in a crossover; any torque component perpendicular to $\mathbf{J}$ averages to zero during the grain rotation.
} 
is ineffective at aligning grains.

Lazarian \& Draine (1997) pointed out that thermal fluctuations in a grain can play an important role in crossovers. In the process of Barnett dissipation, rotational energy is transferred to the lattice vibrational modes, which act as a thermal reservoir. Energy can also move in the opposite direction; a Barnett fluctuation is the spontaneous transfer of some amount of energy from the vibrational modes to the grain rotation (at constant $\mathbf{J}$ ). Since the rotational energy is a function of the angle $\gamma$ between $\hat{\mathbf{a}}_{1}$ and $\mathbf{J}{ }^{4}{ }^{4}$ thermal fluctuations ensure that $\hat{\mathbf{a}}_{1}$ is never perfectly aligned with $\mathbf{J}$, even when the grain is rotating suprathermally. Lazarian \& Draine found that the resulting small amount of disalignment during periods of suprathermal rotation decreases the degree of disalignment that occurs during a crossover. Essentially, the initial disalignment limits the minimum value that $\mathbf{J}$ assumes during the crossover, and thus limits the effect that random gas atom impacts can have on a grain. Lazarian \& Draine concluded that ordinary paramagnetic dissipation can indeed align interstellar grains.

Lazarian \& Draine (1999a) identified another potentially important consequence of Barnett fluctuations, which they called "thermal flipping". Barnett fluctuations cause the grain to undergo a random walk in the angle $\gamma$, and if the fluctuations are strong enough then the grain can flip. ${ }^{5}$ Once a grain that is entering a crossover has flipped, the component of the systematic torque along $\mathbf{J}$ is parallel to $\mathbf{J}$, rather than anti-parallel to $\mathbf{J}$, and the grain is now spun up rather than down. If a thermal flip occurs before $J$ becomes very small, then disalignment can largely be avoided. In the Spitzer \& McGlynn (1979) crossover scenario, $J$ goes to zero during the grain's flip; in the Lazarian \& Draine (1999a) scenario, the grain can flip at an earlier stage and bypass the disaligning, low- $J$ stage. ${ }^{6}$

Lazarian \& Draine (1999a) pointed out that if a grain can thermally flip once, then perhaps it can do so twice. Indeed, if the Barnett fluctuations are strong enough, then the grain could rapidly flip back and forth. If the systematic torque (e.g., due to $\mathrm{H}_{2}$ formation) is fixed in grain body coordinates, then the torque reverses in inertial coordinates each time the grain flips. With rapid flipping, the systematic torque will time-average to zero, and the grain is prevented from being spun up to suprathermal rotation! Lazarian \& Draine refer to this condition as "thermal trapping". They found that grains smaller than a critical size $a_{c} \sim 0.01-0.1 \mu \mathrm{m}$ become thermally trapped, which could explain the observation that relatively small grains are not aligned.

Thus far, all paramagnetic effects had been assumed to be due to electrons. Lazarian \& Draine

\footnotetext{
${ }^{4}$ For an axisymmetric grain with specified angular momentum and rotational energy, the angle $\gamma$ is constant. The torque-free motion of a grain with arbitrary shape is more complicated; see $\S 2.5$.

${ }^{5}$ For an axisymmetric grain, a flip occurs when $(\pi / 2-\gamma)$ changes sign. See $\S 2.5 .2$ for a description of flips in the general case of arbitary grain shape.

${ }^{6}$ Of course, a flip can also occur as a result of the random torques due to gas atom impacts. However, the timescale for such flips to occur is much longer than the timescale for flips due to Barnett fluctuations. Furthermore, such flips only occur during a disaligning, low- $J$ stage.
} 
(1999b) found that for relatively low rotational speeds (as encountered during crossovers), nuclear paramagnetism can be much more important in Barnett dissipation and fluctuations. Taking this into account, Lazarian \& Draine $(1999 \mathrm{~b})$ revised their estimate of the critical trapping grain size to $a_{c} \gtrsim 1 \mu \mathrm{m}$. Grains smaller than $\sim 1 \mu \mathrm{m}$ are then expected to not be aligned, in conflict with the observation that grains with $a \gtrsim 0.1 \mu \mathrm{m}$ are well aligned.

A resolution of this problem can be sought in another line of development in grain alignment theory. Harwit $(1970 \mathrm{a}, \mathrm{b})$ pointed out that absorption and emission of photons could lead to a random walk in the grain angular momentum, with a tendency for the grain angular momentum to be parallel to the Galactic plane. Dolginov (1972) observed that individual grains might have chirality, with different absorption and scattering cross sections for left- and right-handed circularly polarized light, so that anisotropic starlight might exert a systematic torque on a grain.

In the first paper of this series (Draine \& Weingartner 1996, hereafter Paper I), we evaluated the radiative torque (due to the absorption and scattering of starlight) exerted on an irregularly shaped grain with the optical properties of "astronomical silicate" (Draine \& Lee 1984). We found that if the interstellar radiation field is anisotropic at a level of $\sim 10 \%,{ }^{7}$ then radiative torques can dominate $\mathrm{H}_{2}$ formation torques for grains with $a \gtrsim 0.1 \mu \mathrm{m}$. Radiative torques are not effective at spinning up $a \lesssim 0.05 \mu \mathrm{m}$ grains in regions where $\mathrm{H}$ is neutral; the strong opacity of the gas beyond the Lyman limit denies the small grains short-wavelength radiation with which they can strongly couple.

Radiative torques have clear advantages over $\mathrm{H}_{2}$ formation torques as an agent for driving long-lived suprathermal spin-up in the Purcell alignment scenario. First, radiative torques depend on the global grain geometry and the starlight anisotropy direction, which are expected to be stable for $\gtrsim 10^{7} \mathrm{yr}$, longer than the timescale for grain resurfacing. Thus, spindowns and crossovers might occur less frequently if radiative torques are responsible for suprathermal rotation. Still, even one crossover can be deadly if thermal trapping occurs. Since radiative torques depend on the starlight anisotropy direction, which is fixed in space rather than in grain body coordinates, the radiative torque vector is not fixed in body coordinates, and therefore does not exactly reverse in inertial coordinates each time the grain flips. Thus, grains spun up by radiative torques might be impervious to thermal trapping.

Radiative torques have even greater potential; in the second paper of this series (Draine \& Weingartner 1997, hereafter Paper II), we showed that they can directly align grains. In fact, we found that direct alignment by radiative torques likely dominates paramagnetic dissipation for grains with $a \gtrsim 0.1 \mu \mathrm{m}$. In order to simplify the equations describing the grain dynamics, we assumed in Paper II that (1) $\hat{\mathbf{a}}_{1}$ is always parallel to $\mathbf{J}$. We also assumed that (2) the timescale for $\mathbf{J}$ to precess about $\mathbf{B}$ is much shorter than the other timescales involved in the dynamics, so

\footnotetext{
${ }^{7}$ Weingartner \& Draine (2001) have estimated that the visible/UV radiation in the solar neighborhood is $\sim 10 \%$ anisotropic.
} 
that we could immediately average over the precession in the dynamical equations. Given these assumptions, the dynamical state of the grain can be completely specified by the angular speed $\omega$ and the angle $\xi_{\text {II }}$ between $\hat{\mathbf{a}}_{1}$ and $\mathbf{B}$. We constructed "trajectory maps" for three irregularly shaped grains (with $a=0.2 \mu \mathrm{m}$ and astronomical silicate composition) showing the evolution of grains initially characterized by arbitrary combinations of $\left(\omega, \xi_{\mathrm{II}}\right)$. The trajectories often terminate on stable stationary points characterized by a particular $\left(\omega, \xi_{\mathrm{II}}\right)$; i.e., the radiative torques often result in grain alignment. The trajectories also often pass through $\omega=0$, suggesting that crossovers (of a sort) occur naturally in the radiative torque alignment scenario.

However, because of the simplifying assumptions adopted in Paper II, we were unable to follow the dynamics through these low- $J$ stages. First, we ignored the stochastic nature of the torques due to $\mathrm{H}_{2}$ formation and collisions with gas atoms. This stochasticity can be safely ignored during periods of suprathermal rotation but should be included when $\omega \rightarrow 0$. Second, the assumption that $\hat{\mathbf{a}}_{1} \| \mathbf{J}$ breaks down when $J \rightarrow 0$. We would expect this simplification to be more severe, since thermal flipping might prevent $\omega$ from becoming so small that the first simplification fails badly.

Here we generalize the treatment of Paper II by relaxing the assumption that $\hat{\mathbf{a}}_{1} \| \mathbf{J}$. In future papers, we will add a treatment of the stochastic nature of gas atom impacts and $\mathrm{H}_{2}$ formation and we will use the resulting formalism to conduct a systematic study of grain alignment by radiative torques. The outline of the present paper is as follows:

In $\S 2$, we describe the general dynamics of an asymmetric grain and how it responds to external torques. Most studies of grain alignment adopt axisymmetric grain shapes for simplicity, but in this case the high degree of symmetry suppresses the radiative torques. Thus, we treat the most general grain shape, with no degeneracy in the eigenvalues of the inertia tensor. We explain the concept of "flip state" for the grain, and show that the grain can change flip states only when the rotational kinetic energy passes through a critical value. Because the tumbling motion of the grain is very rapid, it is necessary and appropriate to average over the torque-free motion before considering the effects of any external torques. The torque-free motion includes exchange of energy between rotational and vibrational modes through the phenomenon of "Barnett dissipation".

In $\S 3$, we discuss the various external torques that act on interstellar grains, due to the Barnett magnetic moment, gas and IR emission drag, $\mathrm{H}_{2}$ formation, paramagnetic dissipation, and starlight.

The rapid exchange of energy between rotational and vibrational modes implies that even when the grain has a fixed angular momentum $\mathbf{J}$, it is important to average over the different possible values of the grain kinetic energy; this thermal averaging is discussed in $\S 4$. In $\S 5$, we obtain the equations for the time evolution of the angular momentum $J$ and the angle $\xi$ between $\mathbf{J}$ and $\mathbf{B}$.

As already noted, the grain dynamics involves the possibility that thermal fluctuations will cause the grain to change from one "flip state" to the other. In $\S 6$, we develop an algorithm for evolving the grain dynamics, including stochastic flipping. We present an algorithm that can be applied both in the limit where flipping occurs many times even for the shortest feasible computational time step, as well as in the limit where flipping occurs very rarely. 
In $\S 7$, we present results of the dynamical evolution for one particular case (i.e., for a given grain shape, composition, and size; angle $\psi$ between the magnetic field and the radiation anisotropy direction; and radiation field spectrum). For this case, the analysis of Paper II yields two stable stationary points and two crossover points. Applying the more complete analysis developed here, we find a new stable stationary point with a thermal rotational speed and no crossovers. All of the trajectories terminate on either the new stationary point or on one of the two stable stationary points with suprathermal rotation.

In $\S 8$, we summarize the results of this paper and discuss the remaining issues that must be addressed to complete our understanding of grain alignment by radiative torques.

Since we employ a large number of physical quantities, we provide a glossary of notation (Appendix E) for easy reference.

\section{Grain Dynamics}

\subsection{Grain Geometry}

We consider the irregular grain shape from Paper I ("shape 1" in Paper II). The moment of inertia tensor has eigenvalues $I_{1} \geq I_{2} \geq I_{3}$, with principal axes $\hat{\mathbf{a}}_{1}$, $\hat{\mathbf{a}}_{2}$, and $\hat{\mathbf{a}}_{3}$. We define dimensionless parameters $\alpha_{j}$ by

$$
I_{j} \equiv \alpha_{j} \frac{2}{5} \rho V a^{2}
$$

where $\rho$ is the density and $V=4 \pi a^{3} / 3$ is the grain volume.

\subsection{Coordinate Systems}

Since we are interested in grain alignment with the magnetic field $\mathbf{B}$, we adopt a coordinate system $x_{\mathrm{B}}, y_{\mathrm{B}}, z_{\mathrm{B}}$, with $\mathbf{B} \| \hat{\mathbf{z}}_{\mathrm{B}}$. This system, which we call "alignment" coordinates, defines an inertial reference frame. It will also be convenient to define "angular momentum" coordinates $x_{\mathrm{J}}$, $y_{\mathrm{J}}, z_{\mathrm{J}}$, with $\hat{\mathbf{z}}_{\mathrm{J}} \| \mathbf{J}$. We adopt the following transformation between these coordinate systems:

$$
\begin{gathered}
\hat{\mathbf{x}}_{\mathrm{B}}=\cos \xi \cos \phi \hat{\mathbf{x}}_{\mathrm{J}}-\sin \phi \hat{\mathbf{y}}_{\mathrm{J}}+\sin \xi \cos \phi \hat{\mathbf{z}}_{\mathrm{J}} \\
\hat{\mathbf{y}}_{\mathrm{B}}=\cos \xi \sin \phi \hat{\mathbf{x}}_{\mathrm{J}}+\cos \phi \hat{\mathbf{y}}_{\mathrm{J}}+\sin \xi \sin \phi \hat{\mathbf{z}}_{\mathrm{J}} \\
\hat{\mathbf{z}}_{\mathrm{B}}=-\sin \xi \hat{\mathbf{x}}_{\mathrm{J}}+\cos \xi \hat{\mathbf{z}}_{\mathrm{J}},
\end{gathered}
$$

where $\xi$ and $\phi$ are, respectively, the polar and azimuthal angles of $\mathbf{J}$ in alignment coordinates. Thus, we have the correspondence $\hat{\mathbf{x}}_{J}=\hat{\boldsymbol{\xi}}, \hat{\mathbf{y}}_{J}=\hat{\boldsymbol{\phi}}, \hat{\mathbf{z}}_{J}=\hat{\mathbf{J}}$. See Figure 1 .

For computing radiative torques, we define "scattering" coordinates $e_{1}, e_{2}, e_{3}$, with $\hat{\mathbf{e}}_{1}$ parallel to the radiation propagation direction. Three angles are required to specify the grain orientation 
in this frame. Again, see Figure 1. The orientation of $\hat{\mathbf{a}}_{1}$ is described by the two angles $\Theta \in[0, \pi]$ and $\Phi \in[0,2 \pi]$, where

$$
\hat{\mathbf{a}}_{1}=\cos \Theta \hat{\mathbf{e}}_{1}+\sin \Theta \cos \Phi \hat{\mathbf{e}}_{2}+\sin \Theta \sin \Phi \hat{\mathbf{e}}_{3} \quad .
$$

A third angle $\beta \in[0,2 \pi]$ describes rotation of $\hat{\mathbf{a}}_{2}$ about $\hat{\mathbf{a}}_{1}$ :

$$
\hat{\mathbf{a}}_{2}=-\sin \Theta \cos \beta \hat{\mathbf{e}}_{1}+(\cos \Theta \cos \Phi \cos \beta-\sin \Phi \sin \beta) \hat{\mathbf{e}}_{2}+(\cos \Theta \sin \Phi \cos \beta+\cos \Phi \sin \beta) \hat{\mathbf{e}}_{3} .
$$

Given $\hat{\mathbf{a}}_{i}$ and $\hat{\mathbf{e}}_{i}$, the angles $\Theta, \Phi$, and $\beta$ can be found as follows:

$$
\begin{gathered}
\Theta=\cos ^{-1}\left(\hat{\mathbf{a}}_{1} \cdot \hat{\mathbf{e}}_{1}\right), \\
\Phi=2 \tan ^{-1}\left(\frac{\sin \Theta-\hat{\mathbf{a}}_{1} \cdot \hat{\mathbf{e}}_{2}}{\hat{\mathbf{a}}_{1} \cdot \hat{\mathbf{e}}_{3}}\right),
\end{gathered}
$$

and

$$
\beta=2 \tan ^{-1}\left[\frac{\sin \Theta+\hat{\mathbf{a}}_{2} \cdot \hat{\mathbf{e}}_{1}}{\sin \Theta\left(\hat{\mathbf{a}}_{2} \cdot \hat{\mathbf{e}}_{3} \cos \Phi-\hat{\mathbf{a}}_{2} \cdot \hat{\mathbf{e}}_{2} \sin \Phi\right)}\right] .
$$

In Appendix A, we give expressions for $\Phi$ and $\beta$ when the denominators in equations (8) and (9) are zero.

\subsection{Thermal Rotation}

In the absence of (a) internal dissipation and (b) any external torques besides those due to elastic collisions with gas atoms, the rms rotation rate for a sphere of radius $a$ is given by

$$
\omega_{\mathrm{T}}=\left(\frac{15 k T_{\mathrm{gas}}}{8 \pi \rho a^{5}}\right)^{1 / 2}=1.66 \times 10^{5}\left(\frac{3 \mathrm{~g} \mathrm{~cm}^{-3}}{\rho}\right)^{1 / 2}\left(\frac{T_{\mathrm{gas}}}{100 \mathrm{~K}}\right)^{1 / 2}\left(\frac{0.1 \mu \mathrm{m}}{a}\right)^{5 / 2} \mathrm{~s}^{-1}
$$

\subsection{Dynamical Equations}

The equations describing the evolution of $\mathbf{J}$ and the rotational kinetic energy $E$ with time are

$$
\frac{d \mathbf{J}}{d t}=\mathbf{\Gamma}
$$

and

$$
\frac{d E}{d t}=\frac{1}{2}\left(\mathbf{J} \cdot \frac{d \boldsymbol{\omega}}{d t}+\boldsymbol{\Gamma} \cdot \boldsymbol{\omega}\right)
$$

where $\boldsymbol{\Gamma}$ is the net torque on the grain. Barnett dissipation and fluctuations are manifested in the $\mathbf{J} \cdot d \boldsymbol{\omega} / d t$ term. It is convenient to introduce the following dimensionless quantity:

$$
q \equiv 2 I_{1} E / J^{2}
$$


Since the rotational energy lies between $J^{2} / 2 I_{1}$ and $J^{2} / 2 I_{3}, 1 \leq q \leq I_{1} / I_{3}$. The dynamical equation for $q$ is

$$
\frac{d q}{d t}=\frac{1}{J^{2}}\left[I_{1}\left(\mathbf{J} \cdot \frac{d \boldsymbol{\omega}}{d t}+\boldsymbol{\Gamma} \cdot \boldsymbol{\omega}\right)-2 q \mathbf{J} \cdot \boldsymbol{\Gamma}\right]
$$

Generally, $\boldsymbol{\omega}$ is not parallel to $\mathbf{J}$, in which case $\boldsymbol{\omega}$ executes some sort of periodic motion in grain body coordinates and the grain executes a more complicated quasi-periodic motion in space (see $§ \S 2.5 .1$ and 2.5.3). The timescale for this motion is usually much shorter than the timescale on which $\mathbf{J}$ changes due to the external torques (also $\omega^{-1}$ is usually much shorter than this latter timescale). Thus, we will average equations (11) and (14) over one "cycle" of torque-free motion.

We expect the terms $\boldsymbol{\Gamma} \cdot \boldsymbol{\omega}$ and $-2 q \mathbf{J} \cdot \boldsymbol{\Gamma} / I_{1}$ in equation (14) to be of the same order of magnitude, and to be small compared with the rate at which the rotational energy varies due to internal processes, so that only internal relaxation need be considered in determining the evolution of $q$. (We use the term "relaxation" to refer to both dissipation and fluctuations.) We will examine the validity of this approximation on a torque-by-torque basis in $\S 3$.

\subsection{Torque-free Motion}

\subsubsection{Euler Equations}

The motion of an asymmetric rigid body, when the net external torque is zero, is nicely described in $\S 37$ of Landau \& Lifshitz (1976). First, the Euler equations are solved for the components of the angular velocity $\boldsymbol{\omega}$ in grain body coordinates. The solution depends on the magnitude of the angular momentum $\mathbf{J}$ and the rotational kinetic energy $E$, and involves the Jacobi elliptic functions. There are several different notational conventions for these functions in the literature; thus, we begin by defining ours. The elliptic integral of the first kind is given by

$$
F(\epsilon, m) \equiv \int_{0}^{\epsilon} d \theta\left(1-m \sin ^{2} \theta\right)^{-1 / 2}
$$

The Jacobi amplitude $\operatorname{am}(w, m)$ is defined as the inverse of the above integral; if $w=F(\epsilon, m)$, then $\epsilon=\operatorname{am}(w, m)$. The Jacobi elliptic functions are given by $\operatorname{sn}(w, m)=\sin \epsilon, \operatorname{cn}(w, m)=\cos \epsilon$, and $\operatorname{dn}(w, m)=\left(1-m \sin ^{2} \epsilon\right)^{1 / 2}$.

Next, we introduce the dimensionless quantity

$$
k^{2} \equiv \frac{\left(I_{2}-I_{3}\right)(q-1)}{\left(I_{1}-I_{2}\right)\left(1-I_{3} q / I_{1}\right)} .
$$

When $q<I_{1} / I_{2}, k^{2}<1$ and the solution of the Euler equations is as follows:

$$
\omega_{1}= \pm \frac{J}{I_{1}}\left[\frac{I_{1}-I_{3} q}{I_{1}-I_{3}}\right]^{1 / 2} \operatorname{dn}\left(\tau, k^{2}\right)
$$




$$
\begin{aligned}
& \omega_{2}=-\frac{J}{I_{2}}\left[\frac{I_{2}(q-1)}{I_{1}-I_{2}}\right]^{1 / 2} \operatorname{sn}\left(\tau, k^{2}\right), \\
& \omega_{3}=( \pm)_{1} \frac{J}{I_{3}}\left[\frac{I_{3}(q-1)}{I_{1}-I_{3}}\right]^{1 / 2} \operatorname{cn}\left(\tau, k^{2}\right),
\end{aligned}
$$

where the $( \pm)_{1}$ in equation (19) stands for the same sign chosen in equation (17) and

$$
\tau \equiv t J\left[\frac{\left(I_{1}-I_{2}\right)\left(1-I_{3} q / I_{1}\right)}{I_{1} I_{2} I_{3}}\right]^{1 / 2} .
$$

The period in time $t$ is

$$
P_{t}=4 F\left(\pi / 2, k^{2}\right)\left[\frac{I_{1} I_{2} I_{3}}{\left(I_{1}-I_{2}\right)\left(1-I_{3} q / I_{1}\right)}\right]^{1 / 2} J^{-1}
$$

and the period in the variable $\tau$ is

$$
P_{\tau}=4 F\left(\pi / 2, k^{2}\right)
$$

When $q>I_{1} / I_{2}$,

$$
\begin{gathered}
\omega_{1}= \pm \frac{J}{I_{1}}\left[\frac{I_{1}-I_{3} q}{I_{1}-I_{3}}\right]^{1 / 2} \operatorname{cn}\left(\tau, k^{-2}\right), \\
\omega_{2}=-\frac{J}{I_{2}}\left[\frac{I_{2}\left(1-I_{3} q / I_{1}\right)}{I_{2}-I_{3}}\right]^{1 / 2} \operatorname{sn}\left(\tau, k^{-2}\right) \\
\omega_{3}=( \pm)_{1} \frac{J}{I_{3}}\left[\frac{I_{3}(q-1)}{I_{1}-I_{3}}\right]^{1 / 2} \operatorname{dn}\left(\tau, k^{-2}\right)
\end{gathered}
$$

with

$$
\begin{gathered}
\tau \equiv t J\left[\frac{\left(I_{2}-I_{3}\right)(q-1)}{I_{1} I_{2} I_{3}}\right]^{1 / 2}, \\
P_{t}=4 F\left(\pi / 2, k^{-2}\right)\left[\frac{I_{1} I_{2} I_{3}}{\left(I_{2}-I_{3}\right)(q-1)}\right]^{1 / 2} J^{-1},
\end{gathered}
$$

and

$$
P_{\tau}=4 F\left(\pi / 2, k^{-2}\right),
$$

where $( \pm)_{1}$ in equation (25) stands for the sign choice in equation (23). Of course, when $q=I_{1} / I_{2}$, $\omega_{1}=\omega_{3}=0$ and $\omega_{2}= \pm J / I_{2}$.

In Figure 2, we plot $\omega_{i}$ (with plus signs in eqs. 17, 19, 23, and 25) over two periods for the case with $I_{1}: I_{2}: I_{3}=3: 2: 1$ and several values of $q$.

Note that when $q \rightarrow I_{1} / I_{2}$ from below [above], equations (17) through (19) [(23) through (25)] do not tend towards steady rotation about $\hat{\mathbf{a}}_{2}$. Rather, they tend towards a state that oscillates between steady rotation with $\omega_{2}=+J / I_{2}$ and $\omega_{2}=-J / I_{2}$, with abrupt transitions between these two states of rotation. This is illustrated in Figure 3, where we plot $\omega_{i}$ for $q$ slightly less than $I_{1} / I_{2}$. In Figure 3, "+" denotes the case with plus signs in equations (17) and (19) (the positive flip state with respect to $\hat{\mathbf{a}}_{1}$; see $\left.\S 2.5 .2\right)$ and "-" denotes the case with minus signs in equations (17) and (19) (the negative flip state). Note also that the period $P_{\tau} \rightarrow \infty$ as $q \rightarrow I_{1} / I_{2}$. 


\subsubsection{Grain Flips}

From equations (17) and (23), we see that the grain state is not completely specified by $(\mathbf{J}, q)$. Given $\mathbf{J}$, there are two possible configurations for which a grain can spin about one of its principal axes $\hat{\mathbf{a}}_{i}$ - either $\hat{\mathbf{a}}_{i} \| \mathbf{J}$ and $\omega_{i}>0$ or $\hat{\mathbf{a}}_{i} \|-\mathbf{J}$ and $\omega_{i}<0$. We will call the former configuration the "positive flip state" with respect to axis $\hat{\mathbf{a}}_{i}$ and the latter configuration the "negative flip state" with respect to $\hat{\mathbf{a}}_{i}$. The flip states can be generalized in a natural way to cases for which $\mathbf{J}$ does not lie along a principal axis. When $q<I_{1} / I_{2}$, solutions of the Euler equations with a plus (minus) sign in equation (17) have $\omega_{1}>0\left(\omega_{1}<0\right)$, corresponding to a positive (negative) flip state with respect to axis $\hat{\mathbf{a}}_{1}$. (Also, $\omega_{2}$ and $\omega_{3}$ average to zero.) When $q>I_{1} / I_{2}$, the plus (minus) sign in equation (23) corresponds to a positive (negative) flip state with respect to axis $\hat{\mathbf{a}}_{3}$.

From equations (17), (19), (23), and (25), we see that a grain can evolve smoothly from one flip state to another only when $\omega_{1}=0$ and $\omega_{3}=0$. This only occurs when $q$ passes through $I_{1} / I_{2}$. Figure 3 shows $\omega_{i}$ for the two flip states about $\hat{\mathbf{a}}_{1}$ when $q$ is slightly less than $I_{1} / I_{2}$. When $t / P_{t}=0.25,0.75,1.25$, and 1.75 , the $\omega_{i}$ are nearly identical for the two flip states and the motion is very similar to instantaneous rotation about $\hat{\mathbf{a}}_{2}$. Since $\omega_{1}$ does not quite reach zero when $q<I_{1} / I_{2}$, these similarities are not quite exact.

In Figure 4 we plot $I_{i} \omega_{i} / J$, both for $q$ slightly less than $I_{1} / I_{2}$ (solid curve) and for $q$ slightly greater than $I_{1} / I_{2}$ (dashed curve). ${ }^{8}$ For these curves we have adopted $I_{1}: I_{2}: I_{3}=3: 2: 1$ and positive flip states. Suppose the grain has $q<I_{1} / I_{2}$ at $t=0$ and evolves along the solid curve to $q=I_{1} / I_{2}$ when $t=P_{t} / 4$. At this point, several different outcomes are possible. If $q$ remains exactly equal to $I_{1} / I_{2}$, then $\omega_{1}$ and $\omega_{3}$ remain zero, and $\omega_{2}=-J / I_{2}$. If $q$ continues to increase, so that $q>I_{1} / I_{2}$, then there are two possibilities: 1 . The $\omega_{i}$ emerge along the dashed curves rather than along the solid curves, so that the grain is now in the positive flip state with respect to $\hat{\mathbf{a}}_{3} .2$. $\omega_{1}$ and $\omega_{3}$ emerge along the reflection of the dashed curves through the lines $\omega_{i}=0$, so that the grain is now in the negative flip state with respect to $\hat{\mathbf{a}}_{3}$.

Finally, suppose $q$ reaches a maximum value of $I_{1} / I_{2}$ at $t=P_{t} / 4$ and then decreases. Again there are two possibilities: 1 . The $\omega_{i}$ emerge along the solid curves, so that the grain remains in the positive flip state with respect to $\hat{\mathbf{a}}_{1}$. 2. $\omega_{1}$ and $\omega_{3}$ emerge along the reflection of the solid curves through the lines $\omega_{i}=0$. This is a grain flip; the grain is now in the negative flip state with respect to $\hat{\mathbf{a}}_{1}$ (and the angle $\gamma$ between $\mathbf{J}$ and $\hat{\mathbf{a}}_{1}$ now exceeds $\pi / 2$ ). However, such a flip will occur with infinitesimal probability, since there is infinitesimal probability that the maximum value of $q$ attained during a fluctuation will be exactly $I_{1} / I_{2}$. Real flips will occur in a two-step process, in which the grain configuration evolves across $q=I_{1} / I_{2}$ and then back again.

Recall that as $q \rightarrow I_{1} / I_{2}, P_{t} \rightarrow \infty$. Thus, as $q$ gets very close to $I_{1} / I_{2}$, the evolution in $q$ occurs essentially instantaneously compared with the timescale on which the $\omega_{i}$ change for a given

\footnotetext{
${ }^{8}$ Note that the curve for $I_{2} \omega_{2} / J$ is the same for both cases.
} 
$q$. The evolution to $q=I_{1} / I_{2}$ can occur at any time. As $q$ changes, the curves for the $\omega_{i}$ change, with $\omega_{1}=0$ and $\omega_{3}=0$ when $q=I_{1} / I_{2}$. In the examples in this section, we have chosen to have the evolution to $q=0$ occur when $\omega_{3}=0$ and $\omega_{1}$ is nearly zero for the initial $q$ (when $\left.t=P_{t} / 4\right)$. We made this choice so that only two curves would be needed in Figure 4.

In all cases, when $q$ crosses $q=I_{1} / I_{2}$, the grain is equally likely to emerge in either flip state.

\subsubsection{Motion of Grain Axes}

Next, we need to find the motion of the grain axes in space. The orientation of the grain axes in angular momentum coordinates can be expressed using Eulerian angles $(\alpha, \gamma, \zeta)$. Suppose the grain axes $\hat{\mathbf{a}}_{2}, \hat{\mathbf{a}}_{3}, \hat{\mathbf{a}}_{1}$ are originally lined up with $\hat{\mathbf{x}}_{\mathrm{J}}, \hat{\mathbf{y}}_{\mathrm{J}}, \hat{\mathbf{z}}_{\mathrm{J}}$. Any grain orientation can be obtained by performing the following operations on the grain coordinates: 1 . Rotate through angle $\zeta$ about $\hat{\mathbf{a}}_{1}=\hat{\mathbf{z}}_{\mathrm{J}}$. 2. Rotate through angle $\gamma$ about $\hat{\mathbf{a}}_{2}$. 3. Rotate through angle $\alpha$ about $\hat{\mathbf{a}}_{1}$. (See Fig. 1.)

By resolving $\mathbf{J}$ onto grain axes, we find the following relations for the Eulerian angles $\alpha$ and $\gamma$ :

$$
\begin{aligned}
& \sin \alpha \sin \gamma=I_{2} \omega_{2} / J, \\
& \cos \alpha \sin \gamma=I_{3} \omega_{3} / J
\end{aligned}
$$

and

$$
\cos \gamma=I_{1} \omega_{1} / J
$$

Angles $\alpha$ and $\gamma$ are periodic functions of time, with period $P_{t}$ (eqs. 21 and 27). The remaining Eulerian angle, $\zeta$, can be expressed as a sum of two periodic terms, one with period $P_{t}$, and the other with an incommensurate period. Consequently, the overall torque-free motion is not periodic. However, given a function $A(\alpha, \zeta, \gamma)$, we can still find its average value $\bar{A}(q, \pm)$ over the torque-free motion, as follows:

$$
\bar{A}(q, \pm)=\frac{1}{P_{\tau}} \int_{0}^{P_{\tau}} d \tau \frac{1}{2 \pi} \int_{0}^{2 \pi} d \zeta A(\alpha, \zeta, \gamma)
$$

In equation (32), we have explicitly indicated the dependence of $\bar{A}(q, \pm)$ on $q$ and on the flip state; "+" ("-") indicates the positive (negative) flip state with respect to $\hat{\mathbf{a}}_{1}$ or $\hat{\mathbf{a}}_{3}$, depending on $q$. Of

course, $\bar{A}$ may also depend on other variables (e.g., $\mathbf{J}$ ), depending on which quantity $A$ is being averaged.

\subsubsection{Barnett Dissipation}

A paramagnetic grain in steady rotation (constant $\boldsymbol{\omega}$ ) acquires a magnetization $\chi_{0} \boldsymbol{\omega} / \gamma_{\mathrm{g}}$ (the Barnett effect), where $\chi_{0}$ is the static magnetic susceptibility and $\gamma_{\mathrm{g}}$ is the gyromagnetic ratio of the microscopic magnetic dipoles that are responsible for the grain's paramagnetism $\left(\gamma_{\mathrm{g}}=-g_{\mathrm{e}} \mu_{\mathrm{B}} / \hbar \approx\right.$ $-1.76 \times 10^{7} \mathrm{~s}^{-1} \mathrm{G}^{-1}$ for electrons and $\gamma_{\mathrm{g}}=g_{\mathrm{N}} \mu_{\mathrm{N}} / \hbar \approx 1.3 \times 10^{4} \mathrm{~s}^{-1} \mathrm{G}^{-1}$ for nuclei). Thus, in 
describing the paramagnetic response of a grain, the vector $\mathbf{B}_{\mathrm{BE}} \equiv \boldsymbol{\omega} / \gamma_{\mathrm{g}}$ may be regarded as an effective magnetic field, termed the "Barnett equivalent field" by Purcell (1979).

Purcell noted that when a paramagnetic grain undergoes non-steady rotation, $\mathbf{B}_{\mathrm{BE}}$ is not static in grain-body coordinates; consequently, rotational kinetic energy is dissipated. Purcell assumed that this dissipation rate is the same as the paramagnetic dissipation rate for a static grain exposed to true magnetic fields given by $\boldsymbol{\omega} / \gamma_{\mathrm{g}}$, where $\boldsymbol{\omega}$ is evaluated in the (non-inertial!) reference frame in which the grain is stationary. Purcell considered an axisymmetric grain and ignored the constant component of $\boldsymbol{\omega} / \gamma_{\mathrm{g}}$ lying along the symmetry axis. Lazarian \& Draine (2000) showed that this component cannot be neglected without introducing serious error in the dissipation rate when the grain rotation is very fast. The Purcell prescription (sometimes modified as per Lazarian \& Draine) for obtaining the rate at which rotational energy is dissipated is widely used in the grain alignment literature, but it has never actually been proved to be correct. Although errors are possible in some cases, we expect the prescription to be accurate when applied to the grains that polarize starlight.

Since the angular momentum in the microscopic dipoles is generally a tiny fraction of the total angular momentum of the grain, we make the idealization that Barnett dissipation does not affect the angular momentum associated with the grain rotation; i.e., it affects the value of $q$ but not $\mathbf{J}$. The role of the Barnett effect is to provide a coupling allowing energy exchange (at fixed angular momentum) between the rotational and vibrational degrees of freedom of the lattice.

The Barnett equivalent field for a non-axisymmetric grain is quite complicated. For example, suppose $q<I_{1} / I_{2}$. Equations (17) through (19) reveal that, in this case: (a) The biasing field along $\hat{\mathbf{a}}_{1}$ is not constant; it has a non-sinusoidally oscillating component. (b) The rotating component does not consist of a single field with constant magnitude executing sinusoidal rotation. A correct description of the paramagnetic dissipation rate for this case would be prohibitively complicated.

However, a detailed analysis of the Barnett dissipation rate would not be warranted, because: (a) The paramagnetic properties of interstellar grains are poorly known, so we cannot estimate the dissipation rate with high accuracy. (b) As we will see, the Barnett dissipation timescale is many orders of magnitude shorter than the timescales for some of the other processes that play important roles in the grain dynamics. Thus, we will not strictly follow the evolution of $q$ due to Barnett dissipation and fluctuations, but will instead adopt a simple approximate approach, as described below $(\S 6)$. In this approach, we will simply require an estimate of the Barnett dissipation timescale $\tau_{\text {Bar. }}$.

In estimating $\tau_{\text {Bar }}$, we will consider the case of an axisymmetric (specifically, oblate; i.e., $\left.I_{1}>I_{2}=I_{3}\right)$ grain. We will derive an order of magnitude estimate of $\tau_{\mathrm{Bar}}$ and assume that this estimate also holds for non-axisymmetric grains. For oblate grains, $\mathbf{B}_{\mathrm{BE}}$ consists of a static component $\left(\omega_{\|} / \gamma_{g}\right) \hat{\mathbf{a}}_{1}$ plus a component $\omega_{\perp} / \gamma_{g}$ that rotates in the $\hat{\mathbf{a}}_{1}-\hat{\mathbf{a}}_{2}$ plane, with angular velocity $\omega_{\text {rot }} \hat{\mathbf{a}}_{1}$. Setting $I_{2}=I_{3}$ in equations (17) through (21), we find

$$
\omega_{\|}=\frac{J}{I_{1}} \cos \gamma
$$




$$
\omega_{\perp}=\frac{J}{I_{3}} \sin \gamma
$$

and

$$
\omega_{\mathrm{rot}}=\frac{J\left(I_{1}-I_{3}\right)}{I_{1} I_{3}} \cos \gamma
$$

where $\gamma$ is the (constant) angle between $\mathbf{J}$ and $\hat{\mathbf{a}}_{1}$. The energy dissipation rate is given by

$$
\left(\frac{d E}{d t}\right)_{\mathrm{Bar}}=-V \chi^{\prime \prime} B_{\mathrm{BE}, \mathrm{rot}}^{2} \omega_{\mathrm{rot}}
$$

where $V$ is the grain volume, $\chi^{\prime \prime}$ is the imaginary component of the magnetic susceptibility, and $B_{\mathrm{BE}, \text { rot }} \equiv \omega_{\perp} / \gamma_{g}$ is the rotating component of the Barnett equivalent field.

The susceptibility can be roughly approximated using the solution of the modified Bloch equations derived by Wangsness (1956, his eq. A13):

$$
\chi^{\prime \prime}=\frac{\chi_{0} \omega_{\mathrm{rot}} T_{2}}{1+\left(\omega_{\|}+\omega_{\mathrm{rot}}\right)^{2} T_{2}^{2}+\omega_{\perp}^{2} T_{1} T_{2}}
$$

where $T_{1}$ is the spin-lattice relaxation time and $T_{2}$ is the spin-spin relaxation time. For electron paramagnetism, we take $\chi_{0} T_{2} \sim 10^{-13}\left(15 \mathrm{~K} / T_{d}\right) \mathrm{s}, T_{2} \sim 3 \times 10^{-11}-3 \times 10^{-9} \mathrm{~s}$, and $T_{1} \sim 10^{-6} \mathrm{~s}$ (Draine 1996). For nuclear paramagnetism, we take $\chi_{0} \sim 4 \times 10^{-11}(15 \mathrm{~K} / T), T_{2} \sim 3 \times 10^{-5}$ $3 \times 10^{-4} \mathrm{~s}$, and $T_{1} \sim T_{2}$ (Lazarian \& Draine 1999b).

For an oblate grain,

$$
q=1+\frac{I_{1}-I_{3}}{I_{3}} \sin ^{2} \gamma
$$

From equations (33) through (38), we find that (for $J$ held constant, as we assume to be the case for Barnett dissipation)

$$
\begin{gathered}
\frac{d q}{d t}=-\tau_{\mathrm{Bar}}^{-1}(q-1) \frac{\left(1-q I_{3} / I_{1}\right)}{\left(1-I_{3} / I_{1}\right)} \\
\tau_{\mathrm{Bar}}^{-1} \equiv \frac{2 V \chi_{0} T_{2}}{\gamma_{\mathrm{g}}^{2} D} \frac{J^{2}\left(I_{1}-I_{3}\right)}{I_{1} I_{3}^{3}}=\frac{2 V \chi_{0} T_{2}}{\gamma_{\mathrm{g}}^{2} I_{3} D} \frac{\left(I_{1}-I_{3}\right)}{I_{3}} \omega^{2}
\end{gathered}
$$

where $\omega^{2} \equiv J^{2} /\left(I_{1} I_{3}\right)$ and

$$
D=1+\frac{J^{2}}{I_{3}^{2}}\left(\cos ^{2} \gamma T_{2}^{2}+\sin ^{2} \gamma T_{1} T_{2}\right) \sim 1+\frac{I_{1}}{2 I_{3}} \omega^{2} T_{1} T_{2}
$$

where we have taken $\sin ^{2} \gamma \approx \cos ^{2} \gamma \approx 1 / 2$. Thus,

$$
\tau_{\mathrm{Bar}}^{-1} \sim \frac{2 V \chi_{0} T_{2}}{\gamma_{\mathrm{g}}^{2} I_{3}} \frac{\left(I_{1}-I_{3}\right)}{I_{3}} \frac{\omega^{2}}{1+\left(I_{1} / 2 I_{3}\right) \omega^{2} T_{1} T_{2}} .
$$

The Barnett energy dissipation rate $(d E / d t)_{\text {Bar }} \sim-\left(J^{2} / 2 I_{1}\right) \tau_{\text {Bar }}^{-1}(q-1)\left(1-q I_{3} / I_{1}\right) /\left(1-I_{3} / I_{1}\right)$. Note that $\tau_{\text {Bar }}$ is the exponential decay time for $q-1$ when $q-1 \ll 1$. 
Adding the dissipation rates resulting from both electron and nuclear paramagnetism, we estimate

$$
\begin{aligned}
\tau_{\text {Bar }}^{-1} \sim & 1.7 \mathrm{yr}^{-1} \omega_{5}^{2}\left(\frac{0.1 \mu \mathrm{m}}{a}\right)^{2}\left(\frac{15 \mathrm{~K}}{T_{d}}\right)\left(\frac{3 \mathrm{~g} \mathrm{~cm}^{-3}}{\rho}\right)\left(\frac{I_{1}-I_{3}}{I_{3}}\right)\left[\frac{1}{1+3 \times 10^{-6} \omega_{5}^{2}}+\frac{7 \times 10^{4}}{1+100 \omega_{5}^{2}}\right] \\
\sim & 4.7 \mathrm{yr}^{-1}\left(\frac{\omega}{\omega_{\mathrm{T}}}\right)^{2}\left(\frac{0.1 \mu \mathrm{m}}{a}\right)^{7}\left(\frac{3 \mathrm{~g} \mathrm{~cm}^{-3}}{\rho}\right)^{2}\left(\frac{15 \mathrm{~K}}{T_{d}}\right)\left(\frac{T_{\mathrm{gas}}}{100 \mathrm{~K}}\right)\left(\frac{I_{1}-I_{3}}{I_{3}}\right) \times \\
& {\left[\frac{1}{1+8 \times 10^{-6} p}+\frac{7 \times 10^{4}}{1+280 p}\right], }
\end{aligned}
$$

where $\omega_{5}=\omega / 10^{5} \mathrm{~s}^{-1}$ and

$$
p=\left(\frac{\omega}{\omega_{\mathrm{T}}}\right)^{2}\left(\frac{3 \mathrm{~g} \mathrm{~cm}^{-3}}{\rho}\right)\left(\frac{T_{\mathrm{gas}}}{100 \mathrm{~K}}\right)\left(\frac{0.1 \mu \mathrm{m}}{a}\right)^{5} .
$$

Note the very steep dependence of the dissipation rate on $a$ when the grain rotates thermally (recall eq. 10 for the thermal rotation speed $\omega_{\mathrm{T}}$ ). For large $a$ (for which $p$ is small), we expect dissipation due to nuclear paramagnetism to dominate that due to electron paramagnetism, as pointed out by Lazarian \& Draine (1999b). In Figure 5, we plot $\tau_{\text {Bar }}$ versus $a$ for $\omega=\omega_{\mathrm{T}}$ and assuming $I_{1} \approx 2 I_{3}$.

\section{Torques}

\subsection{Coordinate Transformation}

In order to find values of $\overline{\boldsymbol{\Gamma}}(q, \pm)$ and $\overline{\boldsymbol{\Gamma} \cdot \boldsymbol{\omega}}(q, \pm)$ for the various torques acting on a grain, we need to transform vectors from grain body coordinates to angular momentum coordinates. The transformation is a sequence of three rotations, and is given by the following equations:

$$
\begin{aligned}
& \hat{\mathbf{x}}_{\mathrm{J}}=(\cos \alpha \cos \zeta-\sin \alpha \sin \zeta \cos \gamma) \hat{\mathbf{a}}_{2}-(\sin \alpha \cos \zeta+\cos \alpha \sin \zeta \cos \gamma) \hat{\mathbf{a}}_{3}+\sin \zeta \sin \gamma \hat{\mathbf{a}}_{1}, \\
& \hat{\mathbf{y}}_{\mathrm{J}}=(\cos \alpha \sin \zeta+\sin \alpha \cos \zeta \cos \gamma) \hat{\mathbf{a}}_{2}+(\cos \alpha \cos \zeta \cos \gamma-\sin \alpha \sin \zeta) \hat{\mathbf{a}}_{3}-\cos \zeta \sin \gamma \hat{\mathbf{a}}_{1},
\end{aligned}
$$
and

$$
\hat{\mathbf{z}}_{J}=\sin \alpha \sin \gamma \hat{\mathbf{a}}_{2}+\cos \alpha \sin \gamma \hat{\mathbf{a}}_{3}+\cos \gamma \hat{\mathbf{a}}_{1}
$$

\subsection{Barnett Torque}

The Barnett torque $\boldsymbol{\Gamma}_{\mathrm{B}}=\boldsymbol{\mu}_{\mathrm{Bar}} \times \mathbf{B}$. For a grain in steady rotation, the Barnett magnetic moment is given by

$$
\boldsymbol{\mu}_{\mathrm{Bar}}=\frac{\chi_{0} V}{\gamma_{\mathrm{g}}} \boldsymbol{\omega}
$$

for non-steady rotation, the magnetization is more complicated, as discussed in $§ 2.5 .4$. If we assume that equation (48) holds even for the case of non-steady rotation, then $\overline{\boldsymbol{\Gamma}}_{\mathrm{B}}$ can be found very easily. 
Since $\overline{\boldsymbol{\omega} \times \mathbf{B}}=\overline{\boldsymbol{\omega}} \times \mathbf{B}, \overline{\boldsymbol{\Gamma}}_{\mathrm{B}}=\left(\chi_{0} V / \gamma_{\mathrm{g}}\right) \overline{\boldsymbol{\omega}} \times \mathbf{B}$. Transforming $\boldsymbol{\omega}$ from grain body coordinates to angular momentum coordinates and averaging over $\zeta$, we find that

$$
\overline{\boldsymbol{\omega}}(q)=q \mathbf{J} / I_{1} \quad ;
$$

note that $\overline{\boldsymbol{\omega}}$ does not depend on the flip state. Thus,

$$
\overline{\boldsymbol{\Gamma}}_{\mathrm{B}}(q)=\hat{\phi} q J \Omega_{\mathrm{B}} \sin \xi,
$$

where

$$
\Omega_{\mathrm{B}}=-\frac{\chi_{0} V B}{\gamma_{\mathrm{g}} I_{1}} \approx 25 \mathrm{yr}^{-1}\left(\frac{0.1 \mu \mathrm{m}}{a}\right)^{2}\left(\frac{3 \mathrm{~g} \mathrm{~cm}^{-3}}{\alpha_{1} \rho}\right)\left(\frac{\chi_{0}}{3.3 \times 10^{-4}}\right)\left(\frac{B}{5 \mu G}\right) .
$$

Since $\Omega_{\mathrm{B}} \propto \chi_{0}$, electron paramagnetism dominates nuclear paramagnetism here. A correct treatment of the magnetization for the case of non-steady grain rotation does not significantly modify the above result. For example, for an oblate axisymmetric grain, equation (50) need only be modified by the substitution ${ }^{9}$

$$
q \rightarrow q+(q-1) x \frac{1+x\left(2 \frac{I_{2}}{I_{1}}+\frac{T_{1}}{T_{2}}-1\right)}{1+x^{2}\left[\left(2 \frac{I_{2}}{I_{1}}-1\right)^{2}+\frac{I_{2}(q-1)}{I_{1}-I_{2} q} \frac{T_{1}}{T_{2}}\right]}
$$

where

$$
x \equiv\left(\frac{I_{1}-I_{2} q}{I_{1}-I_{2}}\right)^{1 / 2} \frac{J}{I_{2}} T_{2}
$$

The correction term can be safely ignored since $x$ only becomes significant when the grain rotation is highly suprathermal, in which case $q-1$ is small. Note that the timescale for precession about $\mathbf{B}$ is rapid compared with the timescales for all other relevant processes, except for the grain rotation itself and possibly the Barnett dissipation timescale.

Since $\boldsymbol{\Gamma}_{\mathrm{B}} \cdot \boldsymbol{\omega}=\left(\boldsymbol{\mu}_{\mathrm{Bar}} \times \mathbf{B}\right) \cdot \boldsymbol{\omega}=\left(\boldsymbol{\omega} \times \boldsymbol{\mu}_{\mathrm{Bar}}\right) \cdot \mathbf{B}$, we need to consider the component of $\boldsymbol{\mu}_{\mathrm{Bar}}$ that lags $\boldsymbol{\omega}$ in order to find $\overline{\boldsymbol{\Gamma}_{\mathrm{B}} \cdot \boldsymbol{\omega}}$. The relevant component is $\mu_{\mathrm{Bar}} \sim \chi^{\prime \prime} \omega V / \gamma_{g}$; thus, $\overline{\boldsymbol{\Gamma}_{\mathrm{B}} \cdot \boldsymbol{\omega}} \sim \chi^{\prime \prime} \omega^{2} B V / \gamma_{g}$. This is identical to $(d E / d t)_{\text {Bar }}$ except that the interstellar field $B$ is substituted for the Barnett equivalent field $\omega / \gamma_{g}$. Thus,

$$
\overline{\boldsymbol{\Gamma}_{\mathrm{B}} \cdot \boldsymbol{\omega}} \sim \frac{\gamma_{g, e^{-}} B}{\omega}\left(\frac{d E}{d t}\right)_{\mathrm{Bar}, e^{-}}+\frac{\gamma_{g, \mathrm{nuc}} B}{\omega}\left(\frac{d E}{d t}\right)_{\mathrm{Bar}, \mathrm{nuc}},
$$

where the subscripts " $e$ " and "nuc" denote electron and nuclear paramagnetism, respectively. At the low rotational speeds that may be encountered for large grains during crossovers, the electron term in equation (54) may be comparable to the electron Barnett dissipation rate, but in this case nuclear Barnett dissipation dominates $\overline{\boldsymbol{\Gamma}_{\mathrm{B}} \cdot \boldsymbol{\omega}}$.

\footnotetext{
${ }^{9}$ We used the susceptibilities given by Wangsness (1956, his eqs. A11 through A13) in deriving the substitution $(52)$.
} 


\subsection{Drag}

Collisions with gas atoms will exert a drag torque, which can be written

$$
\overline{\boldsymbol{\Gamma}}_{\mathrm{drag}, \text { gas }} \approx-\mathbf{J} / \tau_{\text {drag, gas }},
$$

where, for a sphere,

$$
\tau_{\text {drag, gas }}=8.72 \times 10^{4} \mathrm{yr}\left(\frac{\rho}{3 \mathrm{~g} \mathrm{~cm}^{-3}}\right)\left(\frac{a}{0.1 \mu \mathrm{m}}\right)\left(\frac{T_{\text {gas }}}{100 \mathrm{~K}}\right)^{1 / 2}\left(\frac{3000 \mathrm{~cm}^{-3} \mathrm{~K}}{n_{\mathrm{H}} T_{\text {gas }}}\right) .
$$

For nonspherical shapes, the drag time will tend to be somewhat larger than given by eq. (56), but only by factors of order $\sim 1.5$. The drag torque will also depend on the orientation of the grain relative to $\mathbf{J}$, but this dependence is quite weak and will be neglected.

There is also a drag torque due to the emission of infrared photons by the grain. This torque has the same form as the gas drag torque but is characterized by a drag timescale

$$
\tau_{\text {drag, em }}=1.1 \times 10^{5} \mathrm{yr}\left\langle Q_{\mathrm{abs}}\right\rangle^{-1}\left(\frac{\rho}{3 \mathrm{~g} \mathrm{~cm}^{-3}}\right)\left(\frac{a}{0.1 \mu \mathrm{m}}\right)^{3}\left(\frac{T_{d}}{15 \mathrm{~K}}\right)^{2}\left(\frac{u_{\mathrm{ISRF}}}{u_{\mathrm{rad}}}\right)
$$

(Paper I). In equation (57), $u_{\mathrm{rad}}$ is the energy density of the incident radiation field (that heats the grain) and $u_{\mathrm{ISRF}}=8.64 \times 10^{-13} \mathrm{erg} \mathrm{cm}^{-3}$ is the energy density in the average interstellar radiation field (ISRF) in the solar neighborhood, as determined by Mezger, Mathis, \& Panagia (1982) and Mathis, Mezger, \& Panagia (1983). The grain absorption cross section is $\pi a^{2} Q_{\text {abs }}(a, \lambda)$ and the angle brackets denote averaging over the incident spectrum.

The total drag timescale is given by

$$
\tau_{\text {drag }}^{-1}=\tau_{\text {drag, gas }}^{-1}+\tau_{\text {drag, em }}^{-1} .
$$

Also,

$$
\overline{\boldsymbol{\Gamma}_{\mathrm{drag}} \cdot \boldsymbol{\omega}}=-I_{1} \overline{\omega^{2}} / \tau_{\mathrm{drag}}
$$

which implies

$$
\frac{\overline{\boldsymbol{\Gamma}_{\mathrm{drag}} \cdot \boldsymbol{\omega}}}{(d E / d t)_{\mathrm{Bar}}} \sim \frac{\tau_{\mathrm{Bar}}}{\tau_{\mathrm{drag}}}
$$

Figure 5 shows $\tau_{\text {drag }}$ versus $a$. In computing $\left\langle Q_{\text {abs }}\right\rangle$, we have assumed spherical grains, using Mie theory and the dielectric functions for silicates from Li \& Draine (2001). The specific energy density

$u_{\lambda}$ for the ISRF is given in Paper I. From Figure 5 we see that $\tau_{\text {Bar }} / \tau_{\text {drag }} \ll 1$ for all grain sizes of interest when $\omega \sim \omega_{\mathrm{T}}$. Thus, $\overline{\boldsymbol{\Gamma}_{\mathrm{drag}} \cdot \boldsymbol{\omega}}$ may be neglected in the evolution of $q$. 


\section{4. $\mathrm{H}_{2}$ Formation}

The $\mathrm{H}_{2}$ formation torque $\boldsymbol{\Gamma}_{\mathrm{H}_{2}}$ has a fixed direction in grain body coordinates. When $q<I_{1} / I_{2}$,

$$
\overline{\boldsymbol{\Gamma}}_{\mathrm{H}_{2}}(q, \pm)= \pm\left(\boldsymbol{\Gamma}_{\mathrm{H}_{2}} \cdot \hat{\mathbf{a}}_{1}\right)\left[\frac{I_{1}-I_{3} q}{I_{1}-I_{3}}\right]^{1 / 2} \frac{\pi}{2 F\left(\pi / 2, k^{2}\right)} \hat{\mathbf{J}}
$$

and

$$
\overline{\boldsymbol{\Gamma}_{\mathrm{H}_{2}} \cdot \boldsymbol{\omega}}(q, \pm)= \pm\left|\overline{\boldsymbol{\Gamma}}_{\mathrm{H}_{2}}\right| J / I_{1}
$$

When $q>I_{1} / I_{2}$,

$$
\overline{\boldsymbol{\Gamma}}_{\mathrm{H}_{2}}(q, \pm)= \pm\left(\boldsymbol{\Gamma}_{\mathrm{H}_{2}} \cdot \hat{\mathbf{a}}_{3}\right)\left[\frac{I_{3}(q-1)}{I_{1}-I_{3}}\right]^{1 / 2} \frac{\pi}{2 F\left(\pi / 2, k^{-2}\right)} \hat{\mathbf{J}}
$$

and

$$
\overline{\boldsymbol{\Gamma}_{\mathrm{H}_{2}} \cdot \boldsymbol{\omega}}(q, \pm)= \pm\left|\overline{\boldsymbol{\Gamma}}_{\mathrm{H}_{2}}\right| J / I_{3}
$$

We estimate the magnitude of the $\mathrm{H}_{2}$ formation torque as (see Paper II)

$$
\Gamma_{\mathrm{H}_{2}}=I_{1} \omega_{\mathrm{H}_{2}} / \tau_{\mathrm{drag}, \text { gas }}
$$

with

$$
\omega_{\mathrm{H}_{2}} \sim 5.2 \times 10^{7} \mathrm{~s}^{-1} f\left(\frac{0.1 \mu \mathrm{m}}{a}\right)^{2}\left(\frac{l}{10 \AA}\right)\left(\frac{E_{\mathrm{H}_{2}}}{0.2 \mathrm{eV}}\right)^{1 / 2}\left[\frac{n(\mathrm{H})}{n_{\mathrm{H}}}\right] ;
$$

$f$ is the fraction of the $\mathrm{H}$ atoms that collide with the grain and depart as $\mathrm{H}_{2}, l^{2}$ is the surface area per $\mathrm{H}_{2}$ formation site on the grain surface, $E_{\mathrm{H}_{2}}$ is the kinetic energy of the departing $\mathrm{H}_{2}$ molecules, and $n(\mathrm{H}) / n_{\mathrm{H}}$ is the fraction of the gas-phase $\mathrm{H}$ that is in atomic form. For a real grain, $\boldsymbol{\Gamma}_{\mathrm{H}_{2}}$ varies in magnitude and direction on a timescale characteristic of changes in the surface sites. As in Paper II, we will ignore this complication and assume that $\boldsymbol{\Gamma}_{\mathrm{H}_{2}}$ does not evolve with time.

Equations (62), (64), and (65) yield

$$
\frac{\overline{\boldsymbol{\Gamma}_{\mathrm{H}_{2}} \cdot \boldsymbol{\omega}}}{(d E / d t)_{\mathrm{Bar}}} \sim \frac{\tau_{\mathrm{Bar}}}{\tau_{\text {drag, gas }}} \frac{\omega_{\mathrm{H}_{2}}}{\omega}
$$

Figure 5 shows that $\tau_{\mathrm{H}_{2}} \equiv \tau_{\mathrm{drag} \text {, gas }} \omega / \omega_{\mathrm{T}} \gg \tau_{\mathrm{Bar}}$ when $\omega \sim \omega_{\mathrm{T}}$, so $\overline{\boldsymbol{\Gamma}_{\mathrm{H}_{2}} \cdot \boldsymbol{\omega}}$ may be neglected in the evolution of $q$.

\subsection{Paramagnetic Dissipation}

For steady grain rotation, the torque due to paramagnetic dissipation is given by (Davis \& Greenstein 1951)

$$
\boldsymbol{\Gamma}_{\mathrm{DG}}=\frac{\chi^{\prime \prime} V}{\omega}(\boldsymbol{\omega} \times \mathbf{B}) \times \mathbf{B}
$$


Lazarian \& Draine (2000) pointed out that $\chi^{\prime \prime}$ is modified by the grain rotation itself. They showed that the dissipation rate for a sample rotating in a weak static field $\mathbf{B}$ is equal to the dissipation rate for a static sample subject to the Barnett equivalent field $\boldsymbol{\omega} / \gamma_{g}$ plus the rotating field $\mathbf{B}$. This yields

$$
\chi^{\prime \prime}=\frac{\chi_{0} \omega T_{2}}{1+\gamma_{\mathrm{g}}^{2} B^{2} \sin ^{2} \xi T_{1} T_{2}} \sim \chi_{0} \omega T_{2}
$$

the second term in the denominator is negligible for the magnetic field strengths encountered in the ISM. Since $\chi_{0} T_{2}$ for electron paramagnetism substantially exceeds that for nuclear paramagnetism, the nuclear contribution may be neglected. Note that equation (69) does not have the same form as equation (37) for $\chi^{\prime \prime}$ in the case of Barnett dissipation. The rotation of the interstellar magnetic field (in the grain's frame) is in the correct sense so as to be in resonance with the Barnett equivalent field lying along $\hat{\mathbf{a}}_{1}$, so the term involving $T_{2}^{2}$ is zero. The rotating component of the Barnett equivalent field, on the other hand, rotates in the wrong direction for resonance.

The situation is much more complicated in the case of non-steady grain rotation, due to the increased complexity of the external field as viewed in the grain's frame. For example, for an axisymmetric grain in non-steady rotation, the external field consists of five separate components: a constant component along $\hat{\mathbf{a}}_{1}$, a component that oscillates along $\hat{\mathbf{a}}_{1}$, and three components that rotate (with different angular speeds) about $\hat{\mathbf{a}}_{1}$. Even the simplest analysis, in which the components of the external field along $\hat{\mathbf{a}}_{1}$ are neglected in comparison with the Barnett equivalent field $\omega_{1} \hat{\mathbf{a}}_{1} / \gamma_{g}$, introduces a complicated dependence on $q$, since the field strengths and angular frequencies of the three rotating components all depend on $q .{ }^{10}$

Since we are primarily concerned with radiative torques in this paper, we will ignore the complicated $q$-dependence of the Davis-Greenstein torque, and simply treat it as if the grain were rotating uniformly about $\hat{\mathbf{a}}_{1}$. In this case,

$$
\overline{\boldsymbol{\Gamma}}_{\mathrm{DG}}=-\frac{J}{\tau_{\mathrm{DG}}} \sin \xi(\hat{\boldsymbol{\xi}} \cos \xi+\hat{\mathbf{J}} \sin \xi),
$$

with

$$
\tau_{\mathrm{DG}}=\frac{2 \alpha_{1} \rho a^{2}}{5 \chi_{0} T_{2} B^{2}} \sim 1.5 \times 10^{6} \mathrm{yr}\left(\frac{\alpha_{1} \rho}{3 \mathrm{~g} \mathrm{~cm}^{-3}}\right)\left(\frac{a}{0.1 \mu \mathrm{m}}\right)^{2}\left(\frac{T_{d}}{15 \mathrm{~K}}\right)\left(\frac{5 \mu \mathrm{G}}{B}\right)^{2}
$$

Since $\overline{\boldsymbol{\Gamma}_{\mathrm{DG}} \cdot \boldsymbol{\omega}} \sim \chi^{\prime \prime} V \omega B^{2}, \overline{\boldsymbol{\Gamma}_{\mathrm{DG}} \cdot \boldsymbol{\omega}}$ is suppressed with respect to $(d E / d t)_{\text {Bar }}$ by a factor $\sim$ $\left(B \gamma_{\mathrm{g}} / \omega\right)^{2}\left(1+\omega^{2} T_{1} T_{2}\right)$, rendering it negligible.

\footnotetext{
${ }^{10}$ It should also be noted that the susceptibilities are not necessarily the same as for the case of a single rotating field component. The modified Bloch equations should be solved with all of the rotating field components included. It seems unlikely that the susceptibilities would be significantly affected when all of the rotating fields are as weak as the interstellar field. However, there could be cases in which the presence of the rotating components of the Barnett equivalent field affects the susceptibilities for the components of the interstellar field.
} 


\subsection{Radiative Torques}

The radiative torque on a stationary grain exposed to a unidirectional radiation field with energy density $u$ can be expressed as (Paper I)

$$
\boldsymbol{\Gamma}_{\mathrm{rad}}=\pi a^{2} u \frac{\tilde{\lambda}}{2 \pi}\left\langle\mathbf{Q}_{\Gamma}(\Theta, \Phi, \beta)\right\rangle,
$$

where a tilde denotes spectral averaging:

$$
\begin{aligned}
u & \equiv \int u_{\lambda} d \lambda, \\
\tilde{\lambda} & \equiv \frac{1}{u} \int \lambda u_{\lambda} d \lambda
\end{aligned}
$$

and

$$
\tilde{\mathbf{Q}}_{\Gamma}(\Theta, \Phi, \beta) \equiv \frac{1}{\tilde{\lambda} u} \int \mathbf{Q}_{\Gamma}(\Theta, \Phi, \beta, \lambda) \lambda u_{\lambda} d \lambda
$$

We approximate the interstellar radiation field by an isotropic component with energy density $\left(1-\gamma_{\mathrm{rad}}\right) u_{\text {rad }}$ plus a unidirectional component with energy density $\gamma_{\mathrm{rad}} u_{\mathrm{rad}}$, propagating at angle

$\psi$ with respect to $\mathbf{B}$. We will assume that the starlight anisotropy direction is in the $\hat{\mathbf{x}}_{\mathrm{B}}-\hat{\mathbf{z}}_{\mathrm{B}}$ plane.

\subsubsection{Unidirectional Component}

We adopt the following transformation between "alignment coordinates" and "scattering coordinates" for the unidirectional component of the radiation field:

$$
\begin{gathered}
\hat{\mathbf{e}}_{1}=-\sin \psi \hat{\mathbf{x}}_{\mathrm{B}}+\cos \psi \hat{\mathbf{z}}_{\mathrm{B}} \\
\hat{\mathbf{e}}_{2}=\cos \psi \hat{\mathbf{x}}_{\mathrm{B}}+\sin \psi \hat{\mathbf{z}}_{\mathrm{B}}, \\
\hat{\mathbf{e}}_{3}=\hat{\mathbf{y}}_{\mathrm{B}} ;
\end{gathered}
$$

see Figure 1.

The unidirectional component of the radiative torque is then given by

$$
\overline{\boldsymbol{\Gamma}}_{\mathrm{rad}}^{\mathrm{uni}}(\xi, \phi, \psi, q, \pm)=\frac{1}{2} \gamma_{\mathrm{rad}} u_{\mathrm{rad}} a^{2} \tilde{\lambda}[F(\xi, \phi, \psi, q, \pm) \hat{\boldsymbol{\xi}}+G(\xi, \phi, \psi, q, \pm) \hat{\boldsymbol{\phi}}+H(\xi, \phi, \psi, q, \pm) \hat{\mathbf{J}}]
$$

where

$$
\begin{aligned}
& F(\xi, \phi, \psi, q, \pm)=-\overline{\tilde{\mathbf{Q}}}_{\Gamma} \cdot \hat{\mathbf{e}}_{1}(\sin \psi \cos \xi \cos \phi+\cos \psi \sin \xi) \\
& +\overline{\tilde{\mathbf{Q}}}_{\Gamma} \cdot \hat{\mathbf{e}}_{2}(\cos \psi \cos \xi \cos \phi-\sin \psi \sin \xi)+\overline{\tilde{\mathbf{Q}}}_{\Gamma} \cdot \hat{\mathbf{e}}_{3} \cos \xi \sin \phi, \\
& G(\xi, \phi, \psi, q, \pm)=\overline{\tilde{\mathbf{Q}}}_{\Gamma} \cdot \hat{\mathbf{e}}_{1} \sin \psi \sin \phi-\overline{\tilde{\mathbf{Q}}}_{\Gamma} \cdot \hat{\mathbf{e}}_{2} \cos \psi \sin \phi+\overline{\tilde{\mathbf{Q}}}_{\Gamma} \cdot \hat{\mathbf{e}}_{3} \cos \phi \quad,
\end{aligned}
$$


and

$$
\begin{aligned}
H(\xi, \phi, \psi, q, \pm)= & \overline{\tilde{\mathbf{Q}}}_{\Gamma} \cdot \hat{\mathbf{e}}_{1}(\cos \psi \cos \xi-\sin \psi \sin \xi \cos \phi) \\
& +\overline{\tilde{\mathbf{Q}}}_{\Gamma} \cdot \hat{\mathbf{e}}_{2}(\sin \psi \cos \xi+\cos \psi \sin \xi \cos \phi)+\overline{\mathbf{Q}}_{\Gamma} \cdot \hat{\mathbf{e}}_{3} \sin \xi \sin \phi
\end{aligned}
$$

Note that equations (80) through (82) are identical to equations (21) through (23) in Paper II, except that here the efficiency factor $\tilde{\tilde{\mathbf{Q}}}_{\Gamma}$ is averaged over the torque-free motion described in $\S 2.5$, whereas in Paper II the efficiency factors are averaged over rotation about $\hat{\mathbf{a}}_{1}$. In order to evaluate $\overline{\tilde{\mathbf{Q}}}_{\Gamma}(\xi, \phi, \psi, q, \pm)$, we need to know the values of $(\Theta, \Phi, \beta)$ that correspond to the Eulerian angles $(\alpha, \zeta, \gamma)$ as the grain executes its torque-free motion. For this, we use equations (7) through (9); expressions for the dot products appearing in these equations are given in Appendix B.

For unidirectional radiation,

$$
\overline{\boldsymbol{\Gamma}_{\mathrm{rad}} \cdot \boldsymbol{\omega}}(\xi, \phi, \psi, q, \pm)=\frac{1}{2} \gamma_{\mathrm{rad}} u_{\mathrm{rad}} a^{2} \tilde{\lambda} \frac{q J}{I_{1}} H(\xi, \phi, \psi, q, \pm)
$$

Thus,

$$
\frac{\overline{\boldsymbol{\Gamma}_{\mathrm{rad}} \cdot \boldsymbol{\omega}}}{(d E / d t)_{\mathrm{Bar}}} \sim \frac{\tau_{\mathrm{Bar}}}{\tau_{\mathrm{rad}}}
$$

where

$$
\tau_{\mathrm{rad}}=250 \mathrm{yr}\left(\frac{\rho}{3 \mathrm{~g} \mathrm{~cm}^{-3}}\right)^{1 / 2}\left(\frac{T}{100 \mathrm{~K}}\right)^{1 / 2}\left(\frac{a}{0.1 \mu \mathrm{m}}\right)^{1 / 2}\left(\frac{10^{-3}}{\gamma_{\mathrm{rad}} H}\right)\left(\frac{u_{\mathrm{ISRF}}}{u_{\mathrm{rad}}}\right)\left(\frac{1.2 \mu \mathrm{m}}{\tilde{\lambda}}\right)\left(\frac{\omega}{\omega_{\mathrm{T}}}\right)
$$

We expect $\gamma_{\mathrm{rad}} H \sim 10^{-3}$ (Paper II). For the ISRF, $\tilde{\lambda}=1.2 \mu \mathrm{m}$. Figure 5 shows that $\tau_{\text {Bar }} / \tau_{\text {rad }} \ll 1$ when $\omega \sim \omega_{\mathrm{T}}$, so $\overline{\boldsymbol{\Gamma}_{\mathrm{rad}} \cdot \boldsymbol{\omega}}$ may be neglected in evolving $q$.

\subsubsection{Isotropic Component}

The torque on a grain exposed to the isotropic component of the radiation field is fixed in grain body coordinates and is given by

$$
\Gamma_{\mathrm{rad}, 0}^{\mathrm{iso}}=\frac{1}{2}\left(1-\gamma_{\mathrm{rad}}\right) u_{\mathrm{rad}} a^{2} \tilde{\lambda} \tilde{\mathbf{Q}}_{\Gamma}^{\text {iso }}
$$

where

$$
\tilde{\mathbf{Q}}_{\Gamma}^{\text {iso }} \cdot \hat{\mathbf{a}}_{i}=\frac{1}{4 \pi} \int_{-1}^{1} d \cos \Theta \int_{0}^{2 \pi} d \beta \tilde{\mathbf{Q}}_{\Gamma}(\Theta, 0, \beta) \cdot \hat{\mathbf{a}}_{i}
$$

As with the $\mathrm{H}_{2}$ formation torque, the components perpendicular to $\mathbf{J}$ average to zero during the grain rotation. Thus,

$$
\overline{\boldsymbol{\Gamma}}_{\text {rad }}^{\text {iso }}(q, \pm)= \pm\left(\boldsymbol{\Gamma}_{\text {rad }, 0}^{\text {iso }} \cdot \hat{\mathbf{a}}_{1}\right)\left[\frac{I_{1}-I_{3} q}{I_{1}-I_{3}}\right]^{1 / 2} \frac{\pi}{2 F\left(\pi / 2, k^{2}\right)} \hat{\mathbf{J}}
$$


when $q<I_{1} / I_{2}$ and

$$
\overline{\boldsymbol{\Gamma}}_{\text {rad }}^{\text {iso }}(q, \pm)= \pm\left(\boldsymbol{\Gamma}_{\text {rad }, 0}^{\text {iso }} \cdot \hat{\mathbf{a}}_{3}\right)\left[\frac{I_{3}(q-1)}{I_{1}-I_{3}}\right]^{1 / 2} \frac{\pi}{2 F\left(\pi / 2, k^{-2}\right)} \hat{\mathbf{J}}
$$

when $q>I_{1} / I_{2}$.

Also,

$$
\overline{\boldsymbol{\Gamma}_{\mathrm{rad}}^{\mathrm{iso}} \cdot \boldsymbol{\omega}}(q, \pm) \sim \pm \frac{1}{2}\left(1-\gamma_{\mathrm{rad}}\right) u_{\mathrm{rad}} a^{2} \tilde{\lambda} \tilde{Q}_{\Gamma}^{\mathrm{iso}} \frac{q J}{I_{1}}
$$

Since $\left(1-\gamma_{\mathrm{rad}}\right) \tilde{Q}_{\Gamma}^{\text {iso }}$ is generally less than $\gamma_{\mathrm{rad}}|H|$, we can neglect $\overline{\boldsymbol{\Gamma}_{\mathrm{rad}}^{\text {iso }} \cdot \boldsymbol{\omega}}$.

\subsubsection{Evaluation of Radiative Torques}

We use the discrete dipole approximation ${ }^{11}$ to compute $\mathbf{Q}_{\Gamma}(\Theta, \Phi, \beta)$ (see Paper I). In this exploratory paper, we consider only shape 1 from Paper I and $a=0.2 \mu \mathrm{m}$. We adopt the dielectric functions for astronomical silicate from Li \& Draine (2001). In order to further reduce the computational demands, we assume a monochromatic spectrum, with $\lambda=1.2 \mu \mathrm{m}(\tilde{\lambda}$ for the ISRF). In a future paper, we will consider a range of grain shapes and sizes and we will fully integrate over the ISRF.

Computations are only performed for $\Phi=0$, since

$\mathbf{Q}_{\Gamma}(\Theta, \Phi, \beta)=\mathbf{Q}_{\Gamma}(\Theta, 0, \beta) \cdot \hat{\mathbf{e}}_{1} \hat{\mathbf{e}}_{1}+\mathbf{Q}_{\Gamma}(\Theta, 0, \beta) \cdot \hat{\mathbf{e}}_{2}\left(\hat{\mathbf{e}}_{2} \cos \Phi+\hat{\mathbf{e}}_{3} \sin \Phi\right)+\mathbf{Q}_{\Gamma}(\Theta, 0, \beta) \cdot \hat{\mathbf{e}}_{3}\left(\hat{\mathbf{e}}_{3} \cos \Phi-\hat{\mathbf{e}}_{2} \sin \Phi\right)$

We ran the code for 33 values of $\Theta$ between 0 and $\pi$ (evenly spaced in $\cos \Theta$ ) and 32 values of $\beta$ evenly spaced between 0 and $2 \pi$.

In Figure 6, we plot $F, G$, and $H$ versus $q$ for the case that $\psi=70^{\circ}, \xi=30^{\circ}$, and $\phi=160^{\circ}$. For isotropic radiation, $\mathbf{Q}_{\Gamma}^{\text {iso }} \cdot \hat{\mathbf{a}}_{1}=1.5 \times 10^{-5}, \mathbf{Q}_{\Gamma}^{\text {iso }} \cdot \hat{\mathbf{a}}_{2}=1.2 \times 10^{-6}$, and $\mathbf{Q}_{\Gamma}^{\text {iso }} \cdot \hat{\mathbf{a}}_{3}=-2.2 \times 10^{-7}$.

\section{Thermal Averages over $q$}

Since the Barnett dissipation timescale $\tau_{\text {Bar }}$ is much shorter than any other relevant timescales (except for the timescale characterizing the torque-free motion), we do not follow the evolution of $q$. Instead, we average over a thermal distribution of $q$-values when evaluating the torques. We consider the following two cases (see $\S 6$ for the motivation of these choices). In the first case, we assume that the grain is in the positive flip state with respect to $\hat{\mathbf{a}}_{1}$ when $q<I_{1} / I_{2}$. When $q \geq I_{1} / I_{2}$, we average over both flip states (with respect to $\hat{\mathbf{a}}_{2}$ when $q=I_{1} / I_{2}$ and with respect

\footnotetext{
${ }^{11}$ We use the code DDSCAT.5a10, which is available at http://astro.Princeton.EDU/ draine/.
} 
to $\hat{\mathbf{a}}_{3}$ when $q>I_{1} / I_{2}$ ). A quantity $A$ averaged in this way is denoted by $\langle A\rangle_{+}$. (We assume that $A$ has already been averaged over torque-free motion and do not include a bar to indicate this averaging.) The second case is identical to the first, except that the grain is in the negative flip state with respect to $\hat{\mathbf{a}}_{1}$ when $q<I_{1} / I_{2}$; such averaging is denoted by $\langle A\rangle_{-}$.

\subsection{Density of States}

In order to take thermal averages over $q$, we need to evaluate the density of states in $q$ (for a grain with constant $J$ ). To this end, we examine the grain's trajectories in phase space. In general, the phase space for a rotating grain has six dimensions: one for each Euler angle plus one for each

conjugate momentum. However, when the angular momentum is specified this reduces to just one angle plus its momentum.

The Lagrangian $L$ is just the kinetic energy:

$$
L=\frac{1}{2} \sum_{i} I_{i} \omega_{i}^{2}=\frac{J^{2} \cos ^{2} \gamma}{2 I_{1}}+\frac{J^{2} \sin ^{2} \alpha \sin ^{2} \gamma}{2 I_{2}}+\frac{J^{2} \cos ^{2} \alpha \sin ^{2} \gamma}{2 I_{3}}
$$

(see eqs. 29 through 31 ). The $\omega_{i}$ are related to the Eulerian angles as follows:

$$
\begin{gathered}
\omega_{1}=\dot{\zeta} \cos \gamma+\dot{\alpha}, \\
\omega_{2}=\dot{\zeta} \sin \alpha \sin \gamma+\dot{\gamma} \cos \alpha
\end{gathered}
$$

and

$$
\omega_{3}=\dot{\zeta} \cos \alpha \sin \gamma-\dot{\gamma} \sin \alpha
$$

Using these relations, we rewrite the Langrangian as

$$
L=\frac{J^{2}}{2 I_{2} I_{3}}\left[I_{3}+\left(I_{2}-I_{3}\right) \cos ^{2} \alpha\right]-\frac{I_{1} I_{2} I_{3} \dot{\alpha}^{2}}{2\left[\left(I_{1}-I_{2}\right) I_{3}+I_{1}\left(I_{2}-I_{3}\right) \cos ^{2} \alpha\right]} .
$$

The conjugate momentum is given by

$$
p_{\alpha}=\frac{\partial L}{\partial \dot{\alpha}}=\frac{-I_{1} I_{2} I_{3} \dot{\alpha}}{\left[\left(I_{1}-I_{2}\right) I_{3}+I_{1}\left(I_{2}-I_{3}\right) \cos ^{2} \alpha\right]} .
$$

Expressing the second term in equation (96) for the Lagrangian in terms of $p_{\alpha}$ and setting $L$ equal to the energy $E$, we find

$$
p_{\alpha}=J\left[\frac{I_{3}\left(I_{1}-I_{2} q\right)+I_{1}\left(I_{2}-I_{3}\right) \cos ^{2} \alpha}{I_{3}\left(I_{1}-I_{2}\right)+I_{1}\left(I_{2}-I_{3}\right) \cos ^{2} \alpha}\right]^{1 / 2} .
$$

Sample phase space trajectories are plotted in Figure 7 for the case that $I_{1}: I_{2}: I_{3}=3: 2: 1$. 
The number of states with energy between $E_{1}$ and $E_{2}$ is proportional to the area in phase space bounded by the trajectories for $E_{1}$ and $E_{2}$. Thus, the number of states with $q$-value between 1 and $q$ is proportional to the following quantity:

$$
s \equiv 1-\frac{2}{\pi} \int_{0}^{\alpha_{1}} d \alpha\left[\frac{I_{3}\left(I_{1}-I_{2} q\right)+I_{1}\left(I_{2}-I_{3}\right) \cos ^{2} \alpha}{I_{3}\left(I_{1}-I_{2}\right)+I_{1}\left(I_{2}-I_{3}\right) \cos ^{2} \alpha}\right]^{1 / 2},
$$

where

$$
\alpha_{1} \equiv\left\{\begin{array}{ll}
\pi / 2 & , q \leq I_{1} / I_{2} \\
\cos ^{-1}\left[\frac{I_{3}\left(I_{2} q-I_{1}\right)}{I_{1}\left(I_{2}-I_{3}\right)}\right]^{1 / 2} & , q>I_{1} / I_{2}
\end{array} .\right.
$$

Note that $s$ ranges from 0 to 1 as $q$ ranges from 1 to $I_{1} / I_{3}$.

Since the density of states in the variable $s$ is constant, we will evaluate thermal averages by integrating over $s$. Figure 8 shows $q$ as a function of $s$ for a grain with $I_{1}: I_{2}: I_{3}=3: 2: 1$ and for shape 1 from Paper I.

\subsection{Thermal Averages for the Torques}

The average of a function $\bar{A}(q, \pm)$ over a thermal distribution of $q$ at constant $J$ is given by

$\langle A\rangle_{ \pm}=\frac{\int_{0}^{s_{c}} d s \bar{A}[q(s), \pm] \exp \left[-q(s) J^{2} / 2 I_{1} k T_{d}\right]+\int_{s_{c}}^{1} d s 0.5\{\bar{A}[q(s),+]+\bar{A}[q(s),-]\} \exp \left[-q(s) J^{2} / 2 I_{1} k T_{d}\right]}{\int d s \exp \left[-q(s) J^{2} / 2 I_{1} k T_{d}\right]}$

where the value of $s$ when $q=I_{1} / I_{2}$ is given by

$$
s_{c}=1-\frac{2}{\pi} \sin ^{-1}\left[\frac{I_{1}\left(I_{2}-I_{3}\right)}{I_{2}\left(I_{1}-I_{3}\right)}\right]^{1 / 2} .
$$

If $\bar{A}(q)$ does not depend on the flip state, then we will simply denote the thermal average over $q$ by $\langle A\rangle$.

The Barnett, drag, and Davis-Greenstein torques are all invariant under a change in flip state. With our simple approximations, the Davis-Greenstein torque does not even depend on $q$, so $\left\langle\boldsymbol{\Gamma}_{\mathrm{DG}}\right\rangle$ is given by equation (70). We also have approximated the Barnett and drag torques as simply proportional to $q$. Thus, $\left\langle\boldsymbol{\Gamma}_{\mathrm{B}}\right\rangle$ is given by equation (50) with $q$ replaced by $\langle q\rangle$ and $\left\langle\boldsymbol{\Gamma}_{\text {drag }}\right\rangle=-\langle q\rangle \mathbf{J} / \tau_{\text {drag. }}$. In Figure 9 we plot $\langle q\rangle$ versus $J^{2} /\left(2 I_{1} k T_{d}\right)$. As $J^{2} /\left(2 I_{1} k T_{d}\right) \rightarrow \infty,\langle q\rangle \rightarrow 1$ and as $J^{2} /\left(2 I_{1} k T_{d}\right) \rightarrow 0,\langle q\rangle \rightarrow \int_{0}^{1} d s q(s)$.

The $\mathrm{H}_{2}$ formation torque does depend on flip state and its thermal average is given by

$$
\left\langle\boldsymbol{\Gamma}_{\mathrm{H}_{2}}\right\rangle_{ \pm}= \pm \frac{I_{1} \omega_{\mathrm{H}_{2}}}{\tau_{\text {drag,gas }}}\left(\hat{\boldsymbol{\Gamma}}_{\mathrm{H}_{\mathbf{2}}} \cdot \hat{\mathbf{a}}_{1}\right) C_{1} \hat{\mathbf{J}}
$$

where $C_{1}=\langle C\rangle$ and

$$
C= \begin{cases}{\left[\frac{I_{1}-I_{3} q}{I_{1}-I_{3}}\right]^{1 / 2} \frac{\pi}{2 F\left(\pi / 2, k^{2}\right)}} & , q<I_{1} / I_{2} \\ 0 & , q \geq I_{1} / I_{2}\end{cases}
$$


Note that there is no contribution to $\left\langle\boldsymbol{\Gamma}_{\mathrm{H}_{2}}\right\rangle_{ \pm}$from $q \geq I_{1} / I_{2}$, since we average over flip states for these $q$, with perfect cancellation. In Figure 9 , we plot $C_{1}$ versus $J^{2} /\left(2 I_{1} k T_{d}\right) ; C_{1} \rightarrow 1$ as $J^{2} /\left(2 I_{1} k T_{d}\right) \rightarrow \infty$.

The thermal average of the torque due to the isotropic component of the radiation field is similar to that for the $\mathrm{H}_{2}$ formation torque:

$$
\left\langle\boldsymbol{\Gamma}_{\mathrm{rad}}^{\mathrm{iso}}\right\rangle_{ \pm}= \pm \frac{1}{2}\left(1-\gamma_{\mathrm{rad}}\right) u_{\mathrm{rad}} a^{2} \tilde{\lambda} \tilde{\mathbf{Q}}_{\Gamma}^{\mathrm{iso}} \cdot \hat{\mathbf{a}}_{1} C_{1} \hat{\mathbf{J}}
$$

The thermal average of the torque due to the unidirectional component of the radiation field requires the thermal averages $\langle F\rangle_{ \pm},\langle G\rangle_{ \pm}$, and $\langle H\rangle_{ \pm}$. It is clear from Figure 6 that these do not go to zero when averaging over flip states. (It is not always the case, however, that $H(q)$ for the two flip states are nearly identical.) In Figure 10 we plot $\langle F\rangle_{ \pm},\langle G\rangle_{ \pm}$, and $\langle H\rangle_{ \pm}$versus $J^{2} /\left(2 I_{1} k T_{d}\right)$ for the case that $\psi=70^{\circ}, \xi=30^{\circ}$, and $\phi=160^{\circ}$.

\section{Equations of Motion}

Suppose that during some time interval $\Delta t$ [during which the grain has angular momentum $\mathbf{J}$ defined by $(J, \xi, \phi)]$, if the grain is in a flip state with respect to $\hat{\mathbf{a}}_{1}$, then the probability that it is in the positive flip state is $f_{+}$, and the probability that it is in the negative flip state is $f_{-}=1-f_{+}$. In this case, the thermal average of a quantity $A$ is given by

$$
\langle A\rangle=\langle A\rangle_{+} f_{+}+\langle A\rangle_{-} f_{-}
$$

Prescriptions for $f_{+}$and $f_{-}$are developed in $\S 6$.

The following equations of motion result from equation (11):

$$
\begin{gathered}
\frac{d \phi}{d t}=\langle q\rangle \Omega_{\mathrm{B}}+\frac{\gamma_{\mathrm{rad}} u_{\mathrm{rad}} a^{2} \tilde{\lambda}\langle G\rangle(\xi, \phi, \psi, J)}{2 J \sin \xi}, \\
\frac{d \xi}{d t}=\frac{\gamma_{\mathrm{rad}} u_{\mathrm{rad}} a^{2} \tilde{\lambda}\langle F\rangle(\xi, \phi, \psi, J)}{2 J}-\frac{\sin \xi \cos \xi}{\tau_{\mathrm{DG}}}
\end{gathered}
$$

and

$$
\begin{aligned}
\frac{d J}{d t}= & \frac{1}{2} \gamma_{\mathrm{rad}} u_{\mathrm{rad}} a^{2} \tilde{\lambda}\langle H\rangle(\xi, \phi, \psi, J)-\frac{J}{\tau_{\mathrm{drag}}} \\
& +\left[\frac{I_{1} \omega_{\mathrm{H}_{2}}}{\tau_{\text {drag,gas }}} \hat{\boldsymbol{\Gamma}}_{\mathrm{H}_{\mathbf{2}}}+\frac{1}{2}\left(1-\gamma_{\mathrm{rad}}\right) u_{\mathrm{rad}} a^{2} \tilde{\lambda} \tilde{\mathbf{Q}}_{\Gamma}^{\text {iso }}\right] \cdot \hat{\mathbf{a}}_{1} C_{1}\left(f_{+}-f_{-}\right)-\frac{J \sin ^{2} \xi}{\tau_{\mathrm{DG}}} .
\end{aligned}
$$

These equations are similar to equations (24) through (26) in Paper II (except note the sign error involving $\Omega_{\mathrm{B}}$ in eqs. 10 and 24 in Paper II). The dependence of $\langle F\rangle,\langle G\rangle$, and $\langle H\rangle$ on $J$ results from the presence of $J$ in equation (101). 
In Figure 11 we plot the dynamical times associated with the terms involving $\langle F\rangle_{+},\langle G\rangle_{+}$, and $\langle H\rangle_{+}\left(\tau_{F}, \tau_{G}\right.$, and $\tau_{H}$, defined in the figure caption), as well as the magnetic precession timescale $\left(\langle q\rangle \Omega_{\mathrm{B}}\right)^{-1}$, the Barnett dissipation timescale $\tau_{\mathrm{Bar}}$, and the thermal flipping timescale $\tau_{\mathrm{tf}}$ (see $\S 6$ ), versus $J /\left(I_{1} \omega_{\mathrm{T}, 1}\right)$, for the case that $T_{d}=15 \mathrm{~K}, \psi=70^{\circ}, \xi=30^{\circ}$, and $\phi=160^{\circ}$. As mentioned in $\S 4, \tau_{\text {Bar }}$ is much shorter than the other timescales (although note that $\tau_{F}, \tau_{G}$, and $\tau_{H}$ are shorter when the entire ISRF is adopted).

Since $\left(\langle q\rangle \Omega_{\mathrm{B}}\right)^{-1}$ is much shorter than $\tau_{F}, \tau_{G}$, and $\tau_{H}$ for all $J^{2} /\left(2 I_{1} k T_{d}\right)$ in Figure 11 (and the radiative torques are generally expected to be more important than the Davis-Greenstein and $\mathrm{H}_{2}$ formation torques; see Paper II), we expect that we will generally be able to average the equations of motion over grain precession. In this case, only equations (108) and (109) remain, and $\langle F\rangle(\xi, \phi, \psi, J)$ and $\langle H\rangle(\xi, \phi, \psi, J)$ are averaged over the motion in $\phi$. Thus, we define

$$
\langle F\rangle_{ \pm}^{\phi}(\xi, \psi, J) \equiv \frac{1}{\tau_{\phi}} \int_{0}^{2 \pi} d \phi\left|\frac{d \phi}{d t}\right|^{-1}\langle F\rangle_{ \pm}(\xi, \phi, \psi, J)
$$

and

$$
\langle H\rangle_{ \pm}^{\phi}(\xi, \psi, J) \equiv \frac{1}{\tau_{\phi}} \int_{0}^{2 \pi} d \phi\left|\frac{d \phi}{d t}\right|^{-1}\langle H\rangle_{ \pm}(\xi, \phi, \psi, J)
$$

where

$$
\tau_{\phi} \equiv \int_{0}^{2 \pi} d \phi|d \phi / d t|^{-1}
$$

When $\tau_{G} \gg\left(\langle q\rangle \Omega_{\mathrm{B}}\right)^{-1}, d \phi / d t$ is nearly independent of $\phi$ and $\langle F\rangle_{ \pm}^{\phi}$ and $\langle H\rangle_{ \pm}^{\phi}$ have the form adopted in Paper II (these were denoted $\bar{F}$ and $\bar{H}$ in Paper II). If $\left|\tau_{G}\right|<\left(\langle q\rangle \Omega_{\mathrm{B}}\right)^{-1}$ and $\tau_{G}(\phi)$ undergoes sign changes, then the grain might not even undergo complete cycles of 0 to $2 \pi$ in $\phi$. However, we expect that this will only occur for $\xi \rightarrow 0$ or $\pi$.

When averaging over the motion in $\phi$, we replace $\langle F\rangle(\xi, \phi, \psi, J)$ and $\langle H\rangle(\xi, \phi, \psi, J)$ in equations (108) and (109) with $\langle F\rangle_{+}^{\phi}(\xi, \psi, J) f_{+}+\langle F\rangle_{-}^{\phi}(\xi, \psi, J) f_{-}$and $\langle H\rangle_{+}^{\phi}(\xi, \psi, J) f_{+}+\langle H\rangle_{-}^{\phi}(\xi, \psi, J) f_{-}$, where the evaluation over the thermal averaging cases is done after the averaging over the motion in $\phi$. When $\tau_{\mathrm{tf}}<\left(\langle q\rangle \Omega_{B}\right)^{-1}$, these evaluations should actually be done in the reverse order, but in that case it would not be possible to construct an interpolation table for $\langle F\rangle_{ \pm}^{\phi}$ and $\langle H\rangle_{ \pm}^{\phi}$ prior to running the evolution code (see $\S 6$ ). Since it is almost always the case that $\tau_{G} \gg\left(\langle q\rangle \Omega_{\mathrm{B}}\right)^{-1}$, this reversal of order will not introduce significant error.

The behavior of $\langle F\rangle_{ \pm}^{\phi}(\xi, \psi, J)$ in the limit $\xi \rightarrow 0$ or $\pi$ is somewhat subtle; see Appendix C for a detailed discussion.

\section{Algorithm for Evolving Dynamics With Thermal Flips as the Only Stochastic Element}

As mentioned in $\S 1$, we expect stochasticity due to random gas atom impacts and $\mathrm{H}_{2}$ formation to be less important than random thermal flipping due to Barnett relaxation. Thus, as 
a first approximation, we will evolve the grain dynamics without taking the former stochasticity into account. In a future paper we will describe a more detailed analysis that accounts for this stochasticity in an approximate way.

In order to ensure a consistent treatment of the stochastic flipping throughout the entire dynamical evolution, we integrate the equations of motion using a constant time step size $\Delta t$. We do not expect that there would be any significant biases if a scheme with a variable step size were employed. However, when we generalize further (in a future paper) to include stochasticity associated with gas atom impacts and $\mathrm{H}_{2}$ formation, we will adopt Langevin equations, for which constant step sizes are preferred. Even with a constant step size, the computer time spent running the evolution code is insignificant compared with the time devoted to the scattering calculations and construction of interpolation tables for $\langle F\rangle_{ \pm}^{\phi}$ and $\langle H\rangle_{ \pm}^{\phi}$.

For sufficiently large $J$, the grain will rarely have $q \geq I_{1} / I_{2}$. In this limit, we can consider the grain to always be in one of the flip states with respect to $\hat{\mathbf{a}}_{1}$, with some probability per unit time $\tau_{\mathrm{tf}}^{-1}$ that a flip will occur. We adopt the simple approximation suggested by Lazarian \& Draine (1999a):

$$
\tau_{\mathrm{tf}}^{-1} \sim \tau_{\mathrm{Bar}}^{-1} \exp \left\{-\frac{1}{2}\left[\left(\frac{J}{J_{0}}\right)^{2}-1\right]\right\}
$$

where

$$
J_{0} \equiv\left(\frac{I_{1} I_{2} k T_{d}}{I_{1}-I_{2}}\right)^{1 / 2}=3.2 \times 10^{-20}\left(\frac{\alpha_{1} \alpha_{2}}{\alpha_{1}-\alpha_{2}}\right)^{1 / 2}\left(\frac{\rho}{3 \mathrm{~g} \mathrm{~cm}^{-3}}\right)^{1 / 2}\left(\frac{T_{d}}{15 \mathrm{~K}}\right)^{1 / 2}\left(\frac{a}{0.1 \mu \mathrm{m}}\right)^{5 / 2} \operatorname{ergs} .
$$

During time interval $\Delta t$, the grain spends some fraction $f_{0}$ of the time in its original flip state, and a fraction $\left(1-f_{0}\right)$ in the opposite flip state. Ideally, we would construct the distribution function for $f_{0}$ and randomly select an $f_{0}$ from this distribution for each time step. However, it is not clear how to construct this distribution function. The expectation value $f_{\text {same }}(\Delta t)$ for the fraction of the time step that the grain will be in its original flip state is easily obtained:

$$
f_{\text {same }}(\Delta t)=\frac{1}{\Delta t} \int_{0}^{\Delta t} P_{\text {same }}(t) d t=\frac{1}{2}\left[1+\frac{\exp \left(-\Delta t / \tau_{\mathrm{tf}}\right) \sinh \left(\Delta t / \tau_{\mathrm{tf}}\right)}{\Delta t / \tau_{\mathrm{tf}}}\right],
$$

where

$$
P_{\text {same }}(\Delta t)=\exp \left(-\Delta t / \tau_{\text {tf }}\right) \cosh \left(\Delta t / \tau_{\text {tf }}\right)
$$

is the probability that the grain is in the same flip state after a time $\Delta t$ (see Appendix $\mathrm{D}$ for the derivation of this result). As a simple approximation, we could set $f_{0}=f_{\text {same }}$ for each time step. This would represent "typical" behavior", but would deny the grain the chance of ever spending a significant period of time in one particular flip state. Such a "run" in one flip state could occur in reality, and could have the important consequence of allowing a grain that would otherwise be stuck in the thermal rotation regime to escape to suprathermal rotation. 
Thus, we adopt the following slightly more complicated prescription. The probability that the grain undergoes no flips during the time step is

$$
P_{0}=\exp \left(-\Delta t / \tau_{\mathrm{tf}}\right)
$$

We take the grain to remain in its original flip state throughout the time step with probability $P_{0}$. If the grain does not remain in its original flip state throughout the time step, then we assume that the grain spends a fraction $f_{s}$ of the step in its original flip state, where $f_{s}$ is determined from $P_{0}+\left(1-P_{0}\right) f_{s}=f_{\text {same: }}$

$$
f_{s}=\frac{f_{\text {same }}-P_{0}}{1-P_{0}}
$$

Thus, if the grain is in the positive flip state at the start of the time step, then $f_{+}=1$ and $f_{-}=0$ with probability $P_{0}$; otherwise, $f_{+}=f_{s}$ and $f_{-}=1-f_{s}$.

The flip state at the start of each time step is a stochastic variable. As mentioned above, we take the grain to remain in its original flip state throughout the entire step with probability $P_{0}$. In this case, the flip state at the start of the next step is taken to be the same as it is in the current step. Otherwise, the flip state at the start of the next step is taken to be the opposite of the flip state at the start of the current step with probability $P_{\mathrm{f}}$, determined from $1-P_{\text {same }}=\left(1-P_{0}\right) P_{\mathrm{f}}$ :

$$
P_{\mathrm{f}}=\frac{1-P_{\text {same }}}{1-P_{0}}
$$

Table 1 summarizes the 3 branches which can be taken on each time step.

In the limit of low $J$, the grain does spend a significant fraction of the time with $q \geq I_{1} / I_{2}$. Furthermore, the grain undergoes rapid flipping in this limit, so that the grain spends half of its time with $q>I_{1} / I_{2}$ in the positive flip state with respect to $\hat{\mathbf{a}}_{3}$ and and half of the time in the negative flip state. This motivates the definition of our two thermal averaging cases, given at the beginning of $\S 4$.

If $\Delta t>\tau_{\phi}$, then we average over motion in $\phi$; otherwise we do not. If $\phi$-averaging mode applied in the previous time step but does not apply in the current step, then a value of $\phi$ is selected randomly at the start of the current time step. Since we expect that $\phi$-averaging mode will always apply, we tabulate $\langle F\rangle_{ \pm}\left[\cos \xi, J^{2} /\left(2 I_{1} k T_{d}\right)\right],\langle H\rangle_{ \pm}\left[\cos \xi, J^{2} /\left(2 I_{1} k T_{d}\right)\right]$, and $\tau_{\phi}\left[\cos \xi, J^{2} /\left(2 I_{1} k T_{d}\right)\right]$ for a given $\psi$ before running the dynamical evolution code and interpolate as needed. If non- $\phi$-averaged quantities are needed at any point, then they are calculated within the evolution code.

\section{Results}

As an example of our new method for evolving the grain dynamics including the effects of thermal fluctuations and thermal flipping, we consider shape $1, a=0.2 \mu \mathrm{m}$, a monochromatic radiation field with $\lambda=\tilde{\lambda}_{\mathrm{ISRF}}=1.2 \mu \mathrm{m}, u_{\mathrm{rad}}=u_{\mathrm{rad}, \mathrm{ISRF}}, \gamma_{\mathrm{rad}}=0.1, T=100 \mathrm{~K}, n_{\mathrm{H}}=30 \mathrm{~cm}^{-3}$, 
$T_{d}=15 \mathrm{~K}, B=5 \mu G, \rho=3 \mathrm{~g} \mathrm{~cm}^{-3}$, and $\psi=70^{\circ}$. Because we adopt a monochromatic radiation field with $\lambda=1.2 \mu \mathrm{m}$ (as opposed to using a realistic spectrum for interstellar starlight), this example will be illustrative of a case where the radiative torques - while still dynamically very important - are relatively weak (corresponding to $a \approx 0.1 \mu \mathrm{m}$ grains with a realistic starlight spectrum).

For simplicity, we ignore the torques due to infrared emission (which for this case is negligible compared to gas drag) and $\mathrm{H}_{2}$ formation. In future work we will ascertain the importance of the $\mathrm{H}_{2}$ formation torque.

In Figure 12, we plot the trajectory map calculated using the method of Paper II, in which it is assumed that $\mathbf{J}$ always lies along $\hat{\mathbf{a}}_{1}$ (parallel or anti-parallel). The dotted (solid) trajectories originate at $J / I_{1} \omega_{\mathrm{T}}=30(0.5)$ and the upper (lower) half-plane is for the positive (negative) flip state.

If we had included this map in Paper II, then it would have looked quite different. In Paper II, $\xi$ was defined as the polar angle of $\hat{\mathbf{a}}_{1}$ in alignment coordinates, whereas here we have defined $\xi$ as the polar angle of $\mathbf{J}$ in alignment coordinates. Henceforward, we will write $\xi_{\text {II }}$ for the angle as defined in Paper II. When the grain is in the positive flip state with respect to $\hat{\mathbf{a}}_{1}, \xi_{\mathrm{II}}=\xi$, but in the negative flip state, $\xi_{\mathrm{II}}=\pi-\xi$. In Paper II, negative flip states were indicated by assigning a negative value to $J$; here $J \equiv|\mathbf{J}|$, which is always positive. Since trajectory maps in Paper II were drawn in terms of $\cos \xi_{\mathrm{II}}$, the lower half of the map in this paper (i.e., below the line $J=0$ ) must be reflected in the line $\cos \xi=0$ in order to look like the maps in Paper II (and vice versa).

Note the near symmetry of the map under reflection in the line $J=0$. The maps in Paper II also appear somewhat symmetric, though to a lesser degree than here. (In the maps in Paper II, the symmetry is with respect to a $180^{\circ}$ rotation.) Evidently the radiative torque changes less under a grain flip for a monochromatic radiation spectrum with $\lambda=1.2 \mu \mathrm{m}$ than for the ISRF (at least for shape 1).

From Figure 12, we see that there are two stable stationary points ("attractors"), with $\cos \xi=1$ and $J / I_{1} \omega_{\mathrm{T}}=17.7$ (14.8) for the positive (negative) flip state. There are also two unstable stationary points ("repellors"), with $\left(J / I_{1} \omega_{\mathrm{T}}, \cos \xi\right)=(8.3,0.76)$ for the positive flip state and $\left(J / I_{1} \omega_{\mathrm{T}}, \cos \xi\right)=(4.2,0.74)$ for the negative flip state.

Crossover points, where the trajectories cross $J=0$, are located at $\cos \xi_{\mathrm{II}}=-1,-0.75,0.77$, and 1. (Those at $\cos \xi_{\mathrm{II}}=-1$ and 1 are "crossover repellors". Only one trajectory passes through each of these crossovers; thus, they are not physically relevant.) Suppose $\mathbf{J}$ really were constrained to lie along $\hat{\mathbf{a}}_{1}$, for all $J$. In this case, a grain could enter the crossover at $\cos \xi_{\text {II }}=-0.75$ from above, i.e., in the positive flip state. It would then emerge in the negative flip state, still with $\cos \xi_{\text {II }}=-0.75$, in which case $\cos \xi=0.75$. The direction of $\hat{\mathbf{a}}_{1}$ remains unchanged during the grain flip, while J flips. In other words, the grain's orientation does not change; it stops spinning, and then begins spinning in the opposite sense. Some of the trajectories emerging from this crossover proceed to the crossover at $\cos \xi_{\mathrm{II}}=0.77(\cos \xi=-0.77)$ and others lead to the attractor at $\cos \xi_{\text {II }}=-1(\cos \xi=1)$. Trajectories emerge from the crossover at $\cos \xi_{\text {II }}=0.77$ with the grain 
in the positive flip state, with some heading towards the crossover at $\cos \xi_{\mathrm{II}}=-0.75$ and others heading towards the stationary point at $\cos \xi_{\mathrm{II}}=1$. Thus, in the terminology of Paper II, this is a "semi-cyclic" map.

The dynamical picture changes in two distinct ways when the formalism developed in this paper is adopted. First, since we no longer enforce $\hat{\mathbf{a}}_{1} \| \mathbf{J}$, the structure of the torques as functions of $J, \xi$, and flip state are different. Thus, even in the absence of thermal flips, we can expect qualitative changes in the structure of the trajectory map at low $J$. Second, it is no longer possible to construct strictly deterministic trajectory maps, because of the random thermal flipping. In order to gauge the importance of these two effects, we will progress in stages, first suppressing the thermal flips.

In Figure 13, we display $\langle F\rangle_{+}^{\phi}(\xi)$ and $\langle H\rangle_{+}^{\phi}(\xi)$ for $J \rightarrow \infty$, for $J \rightarrow 0$, and for an intermediate $J ;\langle F\rangle_{-}^{\phi}(\xi)$ and $\langle H\rangle_{-}^{\phi}(\xi)$ are nearly identical to $\langle F\rangle_{+}^{\phi}(\xi)$ and $\langle H\rangle_{+}^{\phi}(\xi)$ at low $J$. The low- $J$ versions of these functions are quite different from the high- $J$ versions. Since the locations of the stationary points and crossovers depend on the structure of these functions, this implies that the locations of crossovers and low- $J$ stationary points could differ from the locations inferred using the analysis of Paper II. Indeed, there could be crossovers or stationary points not even indicated by the analysis of Paper II. Alternatively, such points found using the Paper II formalism might turn out not to exist after all. In Paper II, we showed that crossovers occur when $\langle F\rangle^{\phi}(\xi)=0$. Since $\langle F\rangle^{\phi}$ only has zeros at $\cos \xi= \pm 1$ as $J \rightarrow 0$, we may expect to no longer find crossovers at $\cos \xi_{\mathrm{II}}=-0.75$ and 0.77 .

Figure 14 is the trajectory map (with $J / I_{1} \omega_{\mathrm{T}}=30$ initially) constructed using the method developed in this paper, except that thermal flipping is prohibited: a grain which begins the trajectory in the positive flip state has $f_{+}=1$ and $f_{-}=0$ throughout (and vice versa for the negative flip state). Since thermal flipping is not included, these are "deterministic" trajectories: they are fully determined once the initial conditions (including flip state) are specified. Note the dramatic change in the map: two new attractors have appeared, at $\left(J / I_{1} \omega_{\mathrm{T}}, \cos \xi\right)=(1.40,-0.57)$ (positive flip state) and $(0.71,-0.49)$ (negative flip state), and the crossovers have disappeared. The map is now non-cyclic.

The low- $J$ attractors arise because $\langle H\rangle_{ \pm}^{\phi}$ are smaller in the low- $J$ limit than in the high- $J$ limit due to orientational averaging - at small $J$, thermal fluctuations allow the grain to spend a large fraction of the time with large angles $\gamma$ between $\mathbf{J}$ and $\hat{\mathbf{a}}_{1}$ (see Fig. 13; curves labelled "b" are for the value of $J$ at which the new attractor appears for the positive flip state). We have found that the low- $J$ attractors remain even if the radiation field intensity is increased by a factor 100 . Since the low- $J$ attractors appear even in the absence of thermal flipping, they clearly are not a thermal trapping effect (Lazarian \& Draine 1999a; see $§ 1$ ). The radiative torque simply drives the grain to a state with low $J$.

When a grain undergoes a thermal flip, $\mathbf{J}$ remains fixed in space (i.e., $J$ and $\xi$ are unchanged) and $\hat{\mathbf{a}}_{1}$ flips. In Figure 12 or 14, the point representing the grain's state is reflected in the line $J=0$ 
when the grain thermally flips. Thus, thermal flips do not carry grains across radiative torqueinduced crossovers. Because of the near symmetry of the trajectory map, if a grain undergoes a thermal flip while approaching one of the low- $J$ attractors, it will in most cases land on a trajectory approaching the other low- $J$ attractor. Similarly, a grain that thermally flips while approaching one of the high- $J$ attractors will usually land on a trajectory approaching the other high- $J$ attractor. Due to the presence of the repellors at $\cos \xi \approx 0.75$, there are some cases where a grain that is approaching one of the low- $J$ attractors can be redirected towards one of the high- $J$ attractors (or vice versa), but $\cos \xi$ must start off quite close to 0.75 for this to happen.

Thus, the description of the grain dynamics using deterministic trajectories is an excellent approximation in this case. This is not necessarily true generically, but relies on the high degree of symmetry in the map. Thermal flipping does, however, change the appearance of the trajectories. For sufficiently small $J$, the grain undergoes rapid flipping, so the trajectory map must be symmetric in reflection about the line $J=0$. In Figure 15, we display trajectories constructed using our full, stochastic evolution algorithm (for $J / I_{1} \omega_{\mathrm{T}}=30$ initially). For trajectories in the upper (lower) half-plane, the grain is initially in the positive (negative) flip state. The expectation values $f_{+}$ and $f_{-}$are not explicitly indicated at any point along the trjactories, but it is clear that $f_{+} \approx 1$ when $J / I_{1} \omega_{\mathrm{T}} \gtrsim 5$ (for trajectories that start in the positive flip state) and $f_{+} \approx f_{-} \approx 0.5$ when $J / I_{1} \omega_{\mathrm{T}} \lesssim 5$. The transition from virtually no flipping to rapid flipping occurs when $J / I_{1} \omega_{\mathrm{T}} \sim 5$, as expected from Figure 11. We have displayed trajectories resulting from one iteration of our stochastic evolution code; other realizations look nearly identical.

The low- $J$ attractors fall in the rapid-flipping regime; the rapid flipping shifts their position to $\left(J / I_{1} \omega_{\mathrm{T}}, \cos \xi\right)=(1.14,-0.50)$. Although Figure 15 gives the impression that there are two low- $J$ attractors, these are really the same, because of the rapid flipping.

In Figure 16, we plot the map for trajectories starting with $J / I_{1} \omega_{\mathrm{T}}=1$. The grain is always in the rapid-flipping regime for the low values of $J$ in Figure 16. All of these trajectories land on the new, low- $J$ attractor (except for physically irrelevant trajectories with $\cos \xi=1$ initially). We have found that when $J / I_{1} \omega_{\mathrm{T}}<2$ initially, all trajectories land on the low- $J$ crossover. Thus, if a population of real interstellar grains were characterized by a trajectory map similar to this one, then the high- $J$ attractors might not be relevant, since the grains are unlikely to be in an initial state of suprathermal rotation (although only a very mild initial suprathermality is needed for some grains to end up at the suprathermal attractors).

We have also analyzed the grain dynamics when $\psi=0$ and $30^{\circ}$. The method of Paper II yields a semi-cyclic map with two suprathermal attractors when $\psi=0$ and a cyclic map when $\psi=30^{\circ}$. In both of these cases, the method developed in this paper yields a non-cyclic map with a low- $J$ attractor. 


\section{Summary}

In Paper II, we derived equations of motion for a grain exposed to radiative torques, under the simplifying assumption that the angular momentum $\mathbf{J}$ is always parallel to the principal axis of greatest moment of inertia $\hat{\mathbf{a}}_{1}$. We found that radiative torques can spin grains up to suprathermal rotational speeds and (in combination with the Barnett torque) align the grains with the interstellar magnetic field. In addition, radiative torques can drive grains into periods of thermal rotation, when they might undergo a type of crossover in which the direction of $\mathbf{J}$ reverses.

Because of internal fluctuations, $\hat{\mathbf{a}}_{1}$ and $\mathbf{J}$ are not necessarily parallel when $J$ is small. Thus, we were unable in Paper II to follow the grain dynamics during periods of thermal rotation. Consequently, we were unable to ascertain the role of crossovers in the dynamics.

Here, we have generalized the treatment of Paper II, relaxing the constraint that $\hat{\mathbf{a}}_{1} \| \mathbf{J}$ and including the effects of thermal fluctuations. Our principal results are:

1. We provide a detailed description of torque-free rotation ( $(2.5)$, considering the most general grain shape, with no degeneracy in the eigenvalues of the inertia tensor. The rotation is completely characterized by the angular momentum $\mathbf{J}$, the rotational energy $E$, and the "flip state" (§2.5.2). For convenience, we define a dimensionless measure of the energy at constant $J, q \equiv 2 I_{1} E / J^{2}$.

2. We derive approximate expressions for the external torques, averaged over torque-free rotation (which occurs on a much shorter timescale than the dynamical timescales associated with the torques), as functions of $\mathbf{J}, q$, and flip state. We consider the torques due to the Barnett magnetic moment, gas and IR emission drag, $\mathrm{H}_{2}$ formation, paramagnetic dissipation, and radiation.

3. We make an order-of-magnitude estimate of the Barnett dissipation rate (§2.5.4) and find that internal dissipation dominates the external torques in determining the evolution of $q$.

4. We derive the density of states in the parameter $q$ and find expressions for the thermal averages of the torques for various cases of interest $(\S 4)$.

5. We develop an algorithm for evolving the grain dynamics (§6). The algorithm allows for random thermal flips.

6. As an illustration of the method, we calculate a trajectory map for one particular case $(\S 7)$. Using the method of Paper II, we find two attractors (characterized by suprathermal rotation) and two crossover points. The more complete treatment developed here yields no crossover points and a third attractor, characterized by thermal rotation and rapid flipping. Thus, there are qualitative differences introduced by the proper inclusion of thermal fluctuations and flipping: whereas the Paper II analysis produces a semi-cyclic map, the new analysis produces a non-cyclic map.

In future work, we will investigate more cases, in an effort to determine whether or not noncyclic maps with low- $J$ attractors are a generic feature when the analysis developed here is applied. For this, it will be crucial to employ the full ISRF, rather than a monochromatic spectrum with 
$\lambda=1.2 \mu \mathrm{m}$. In addition, we will explore the effect of stochastic gas atom impacts and $\mathrm{H}_{2}$ formation events on the dynamics. Ultimately, we will conduct a systematic study of grain alignment by radiative torques, considering a range of shapes and sizes.

This research was supported by NSERC of Canada, NSF grant AST 9988126, and an NSF International Research Fellowship to J. C. W. We are grateful to A. Lazarian for many stimulating discussions.

\section{A. Angles in Scattering Coordinates}

When $\hat{\mathbf{a}}_{1} \cdot \hat{\mathbf{e}}_{3}=0, \Phi=0$ if $\hat{\mathbf{a}}_{1} \cdot \hat{\mathbf{e}}_{2} \geq 0$ and $\Phi=\pi$ if $\hat{\mathbf{a}}_{1} \cdot \hat{\mathbf{e}}_{2}<0$.

When $\sin \Theta=0$,

$$
\beta=2 \tan ^{-1}\left(\frac{1-\hat{\mathbf{a}}_{2} \cdot \hat{\mathbf{e}}_{2} \cos \Theta}{\hat{\mathbf{a}}_{2} \cdot \hat{\mathbf{e}}_{3}}\right)
$$

If $\hat{\mathbf{a}}_{2} \cdot \hat{\mathbf{e}}_{3}=0$, then $\beta=\pi-\Theta$ if $\hat{\mathbf{a}}_{2} \cdot \hat{\mathbf{e}}_{2}=-1$ and $\beta=\Theta$ if $\hat{\mathbf{a}}_{2} \cdot \hat{\mathbf{e}}_{2}=1$.

When $\sin \Theta \neq 0$ and $\hat{\mathbf{a}}_{2} \cdot \hat{\mathbf{e}}_{3} \cos \Phi=\hat{\mathbf{a}}_{2} \cdot \hat{\mathbf{e}}_{2} \sin \Phi, \beta=0$ if $\hat{\mathbf{a}}_{2} \cdot \hat{\mathbf{e}}_{1}<0$ and $\beta=\pi$ if $\hat{\mathbf{a}}_{2} \cdot \hat{\mathbf{e}}_{1}>0$.

\section{B. Dot Products Needed in $§ 3.6 .1$}

$$
\begin{gathered}
\hat{\mathbf{a}}_{i} \cdot \hat{\mathbf{e}}_{1}=\cos \psi \hat{\mathbf{z}}_{\mathrm{B}} \cdot \hat{\mathbf{a}}_{i}-\sin \psi \hat{\mathbf{x}}_{\mathrm{B}} \cdot \hat{\mathbf{a}}_{i}, \\
\hat{\mathbf{a}}_{i} \cdot \hat{\mathbf{e}}_{2}=\cos \psi \hat{\mathbf{x}}_{\mathrm{B}} \cdot \hat{\mathbf{a}}_{i}+\sin \psi \hat{\mathbf{z}}_{\mathrm{B}} \cdot \hat{\mathbf{a}}_{i}, \\
\hat{\mathbf{a}}_{i} \cdot \hat{\mathbf{e}}_{3}=\hat{\mathbf{y}}_{\mathrm{B}} \cdot \hat{\mathbf{a}}_{i},
\end{gathered}
$$

with

$$
\begin{gathered}
\hat{\mathbf{x}}_{\mathrm{B}} \cdot \hat{\mathbf{a}}_{1}=\cos \xi \cos \phi \sin \zeta \sin \gamma+\sin \phi \cos \zeta \sin \gamma+\sin \xi \cos \phi \cos \gamma \\
\hat{\mathbf{z}}_{\mathrm{B}} \cdot \hat{\mathbf{a}}_{1}=\cos \xi \cos \gamma-\sin \xi \sin \zeta \sin \gamma \\
\hat{\mathbf{y}}_{\mathrm{B}} \cdot \hat{\mathbf{a}}_{1}=\cos \xi \sin \phi \sin \zeta \sin \gamma-\cos \phi \cos \zeta \sin \gamma+\sin \xi \sin \phi \cos \gamma \\
\hat{\mathbf{x}}_{\mathrm{B}} \cdot \hat{\mathbf{a}}_{2}=\cos \xi \cos \phi(\cos \alpha \cos \zeta-\sin \alpha \sin \zeta \cos \gamma)-\sin \phi(\cos \alpha \sin \zeta+\sin \alpha \cos \zeta \cos \gamma)+\sin \xi \cos \phi \sin \alpha \sin \gamma \\
\hat{\mathbf{z}}_{\mathrm{B}} \cdot \hat{\mathbf{a}}_{2}=-\sin \xi(\cos \alpha \cos \zeta-\sin \alpha \sin \zeta \cos \gamma)+\cos \xi \sin \alpha \sin \gamma
\end{gathered}
$$




\section{Calculation of $\langle F\rangle_{ \pm}^{\phi}(\xi)$ when $\sin \xi \ll 1$}

When $\sin \xi \ll 1,\left\langle\tilde{\mathbf{Q}}_{\Gamma}\right\rangle_{ \pm}(\xi, \phi) \approx\left\langle\tilde{\mathbf{Q}}_{\Gamma}\right\rangle_{ \pm}(\xi=0$ or $\pi)$ is nearly constant as the grain orbits in $\phi$ and, from the definitions of $F$ and $G$ (eqs. 80 and 81), we can write

$$
\langle F\rangle_{ \pm}(\xi, \phi)=F_{ \pm}^{0} \cos \xi \cos \left(\phi-\phi_{0}\right)-F_{ \pm}^{1} \sin \xi
$$

and

$$
\langle G\rangle_{ \pm}(\xi, \phi)=-F_{ \pm}^{0} \sin \left(\phi-\phi_{0}\right)
$$

where

$$
\begin{gathered}
F_{ \pm}^{0} \equiv\left[\left(\left\langle\tilde{\mathbf{Q}}_{\Gamma}\right\rangle_{ \pm} \cdot \hat{\mathbf{e}}_{1} \sin \psi-\left\langle\tilde{\mathbf{Q}}_{\Gamma}\right\rangle_{ \pm} \cdot \hat{\mathbf{e}}_{2} \cos \psi\right)^{2}+\left(\left\langle\tilde{\mathbf{Q}}_{\Gamma}\right\rangle_{ \pm} \cdot \hat{\mathbf{e}}_{3}\right)^{2}\right]^{1 / 2} \\
F_{ \pm}^{1} \equiv\left\langle\tilde{\mathbf{Q}}_{\Gamma}\right\rangle_{ \pm} \cdot \hat{\mathbf{e}}_{1} \cos \psi+\left\langle\tilde{\mathbf{Q}}_{\Gamma}\right\rangle_{ \pm} \cdot \hat{\mathbf{e}}_{2} \sin \psi
\end{gathered}
$$

and the angle $\phi_{0}$ is determined by

$$
\begin{aligned}
\sin \phi_{0} & =\left\langle\tilde{\mathbf{Q}}_{\Gamma}\right\rangle_{ \pm} \cdot \hat{\mathbf{e}}_{3} / F_{ \pm}^{0} \\
\cos \phi_{0} & =\left(-\left\langle\tilde{\mathbf{Q}}_{\Gamma}\right\rangle_{ \pm} \cdot \hat{\mathbf{e}}_{1} \sin \psi+\left\langle\tilde{\mathbf{Q}}_{\Gamma}\right\rangle_{ \pm} \cdot \hat{\mathbf{e}}_{2} \cos \psi\right) / F_{ \pm}^{0}
\end{aligned}
$$

The equation of motion (107) for $\phi$ becomes

$$
\frac{d \phi}{d t}=\langle q\rangle \Omega_{\mathrm{B}}\left[1-\frac{g}{\sin \xi} \sin \left(\phi-\phi_{0}\right)\right],
$$

where

$$
g \equiv \frac{\gamma_{\mathrm{rad}} u_{\mathrm{rad}} a^{2} \tilde{\lambda}}{2 J\langle q\rangle \Omega_{\mathrm{B}}} F_{ \pm}^{0} \sim 8 \times 10^{-6}\left(\frac{u_{\mathrm{rad}}}{u_{\mathrm{ISRF}}}\right)\left(\frac{\tilde{\lambda}}{1.2 \mu \mathrm{m}}\right)\left(\frac{F_{ \pm}^{0}}{10^{-3}}\right)\left(\frac{a}{0.1 \mu \mathrm{m}}\right)^{3 / 2}\left(\frac{\omega}{\omega_{\mathrm{T}}}\right)^{-1}
$$

in equation (C8), we have taken $\langle q\rangle \approx 1, \gamma_{\mathrm{rad}}=0.1, T_{\text {gas }}=100 \mathrm{~K}, \chi_{0}=3.3 \times 10^{-4}$, and $B=5 \mu \mathrm{G}$.

A transition from periodic motion in $\phi$ to no motion in $\phi$ occurs when $\sin \xi=g$. When $\sin \xi>g$, the motion in $\phi$ is periodic with period

$$
\tau_{\phi}=\frac{2 \pi}{\langle q\rangle \Omega_{\mathrm{B}}}\left[1-\left(\frac{g}{\sin \xi}\right)^{2}\right]^{-1 / 2}
$$

and $\cos \left(\phi-\phi_{0}\right)$ averages to zero over the motion in $\phi$. When $\sin \xi \leq g$, the dynamics is characterized by a stable stationary point $\phi_{s}$ given by

$$
\begin{aligned}
\sin \left(\phi_{s}-\phi_{0}\right) & =\sin \xi / g \\
\cos \left(\phi_{s}-\phi_{0}\right) & =\left[1-(\sin \xi / g)^{2}\right]^{1 / 2}
\end{aligned}
$$

Thus,

$$
\langle F\rangle_{ \pm}^{\phi}(\xi)=\left\{\begin{array}{ll}
-F_{ \pm}^{1} \sin \xi & , g \leq \sin \xi \ll 1 \\
-F_{ \pm}^{1} \sin \xi+F_{ \pm}^{0} \cos \xi\left[1-(\sin \xi / g)^{2}\right]^{1 / 2} & , \sin \xi \leq g, \quad \sin \xi \ll 1
\end{array} .\right.
$$


As discussed in $\S 6$, we construct interpolation tables in $\cos \xi$ and $J^{2} /\left(2 I_{1} k T_{d}\right)$ for $\langle F\rangle_{ \pm}^{\phi}$ and $\langle H\rangle_{ \pm}^{\phi}$, to be used in evolving the grain dynamics. However, we use equation (C12) to directly evaluate $\langle F\rangle_{ \pm}^{\phi}$ when $\xi$ lies within one interpolation zone of 0 or $\pi$. Otherwise, it would be impossible to reproduce the small-sin $\xi$ structure with a reasonable number of interpolation zones. Thus, we also construct a table of $F_{ \pm}^{0}\left[J^{2} /\left(2 I_{1} k T_{d}\right)\right]$. To ensure continuity at $\xi_{1}=$ the first interpolation point away from $\xi=0$ or $\pi$, we estimate $F_{ \pm}^{1} \approx-\langle F\rangle_{ \pm}^{\phi}\left(\xi_{1}\right) / \sin \xi_{1}$.

\section{Derivation of $P_{\text {same }}$}

The probability that exactly $n$ flips occur during $\Delta t$ is

$$
P_{n}=\frac{1}{n !} \kappa^{n} e^{-\kappa}
$$

where $\kappa \equiv \Delta t / \tau_{\mathrm{tf}}$. The probability of zero flips or an even number of flips is

$$
\begin{aligned}
P_{\text {same }}=\sum_{j=0}^{\infty} P_{2 j} & =e^{-\kappa} \sum_{j=0}^{\infty} \frac{\kappa^{2 j}}{(2 j) !} \\
& =e^{-\kappa} \frac{1}{2}\left[\sum_{j=0}^{\infty} \frac{\kappa^{j}}{j !}+\sum_{j=0}^{\infty} \frac{(-\kappa)^{j}}{j !}\right] \\
& =e^{-\kappa} \frac{1}{2}\left(e^{\kappa}+e^{-\kappa}\right)=e^{-\kappa} \cosh (\kappa) .
\end{aligned}
$$




\section{E. Glossary of Notation}

\begin{tabular}{|c|c|}
\hline symbol & significance \\
\hline$a$ & radius of sphere of equal volume, $a=(3 V / 4 \pi)^{1 / 3}$ \\
\hline$\hat{\mathbf{a}}_{1}, \hat{\mathbf{a}}_{2}, \hat{\mathbf{a}}_{3}$ & grain principal axes (eigenvectors of moment of inertia tensor) \\
\hline$I_{1}, I_{2}, I_{3}$ & eigenvalues of moment of inertia tensor $\left(I_{1} \geq I_{2} \geq I_{3}\right)$ \\
\hline $\mathbf{J}$ & angular momentum of grain \\
\hline$\omega_{\mathrm{T}}$ & rotation rate for a sphere in thermal equilibrium with the gas (eq. 10) \\
\hline$q$ & $E /\left(J^{2} / 2 I_{1}\right)($ eq. 13$)$ \\
\hline$T_{d}$ & vibrational temperature of grain \\
\hline$\tau_{\mathrm{Bar}}$ & $\begin{array}{l}\text { at fixed } J \text {, timescale for Barnett damping } \\
\text { of rotational kinetic energy in excess of } J^{2} / 2 I_{1} \text { (eq. } 43 \text { ) }\end{array}$ \\
\hline $\bar{A}(q, \pm)$ & $\begin{array}{l}\text { time-average of dynamical variable } A \text { over torque-free motion } \\
\left.\text { with } E /\left(J^{2} / 2 I_{1}\right)=q \text { and in } \pm \text { flip state (eq. } 32\right)\end{array}$ \\
\hline$\tilde{A}$ & average over radiation field spectrum (eqs. 74,75$)$ \\
\hline$\langle A\rangle_{ \pm}\left(J, T_{d}, \xi, \phi\right)$ & thermal average of dynamical variable $A$ \\
\hline$\langle A\rangle_{ \pm}^{\phi}\left(J, T_{d}, \xi\right)$ & $\begin{array}{l}\text { for grain with given } \mathbf{J} \text { and } T_{d} \text {, in } \pm \text { flip state with respect to } \hat{\mathbf{a}}_{1} \text { (eq. 101) } \\
\text { thermal average of variable } A \text { for grain with given } J \text { and } T_{d} \text {, in } \pm \text { flip } \\
\text { state with respect to } \hat{\mathbf{a}}_{1} \text {, averaged over motion in } \phi(\text { eqs. } 110,111)\end{array}$ \\
\hline$\tau_{\mathrm{tf}}^{-1}$ & probability per unit time that grain will change its flip state (eq. 113) \\
\hline$P_{0}(\Delta t)$ & probability that grain undergoes no flips during interval $\Delta t$ \\
\hline$P_{\text {same }}(\Delta t)$ & $\begin{array}{l}\text { probability that flip state at end of interval } \\
\text { will be same as flip state at beginning of interval (eq. 116) }\end{array}$ \\
\hline$f_{\text {same }}(\Delta t)$ & $\begin{array}{l}\text { expectation value for fraction of time that grain } \\
\text { will spend in initial flip state during interval } \Delta t \text { (eq. 115) }\end{array}$ \\
\hline$f_{\mathrm{s}}(\Delta t)$ & $\begin{array}{l}\text { expectation value for fraction of time spent in original flip state } \\
\text { for grain which flips at least once during interval } \Delta t \text { (eq. 118) }\end{array}$ \\
\hline $\mathbf{Q}_{\Gamma}(\Theta, \Phi, \beta, \lambda)$ & $\begin{array}{l}\text { radiative torque efficiency vector for grain orientation }(\Theta, \Phi, \beta) \\
\text { relative to direction of incident radiation with wavelength } \lambda \text { (eq. 72) }\end{array}$ \\
\hline
\end{tabular}

\section{REFERENCES}

Davis, L. \& Greenstein, J. L. 1951, ApJ, 114, 206

Dolginov, A.Z. 1972, Ap\&SS, 18, 337

Dolginov, A. Z. \& Mytrophanov, I. G. 1976, Ap\&SS, 43, 291

Draine, B. T. 1996, in ASP Conf. Ser. 97, Polarimetry of the Interstellar Medium, ed. W. G. Roberge \& D. C. B. Whittet (San Francisco:ASP), 16 
Draine, B. T. \& Lee, H. M. 1984, ApJ, 285, 89

Draine, B. T. \& Weingartner, J. C. 1996, ApJ, 470, 551 (Paper I)

Draine, B. T. \& Weingartner, J. C. 1997, ApJ, 480, 633 (Paper II)

Hall, J. S. 1949, Science, 109, 166

Hall, J. S., \& Mikesell, A.H. 1949, AJ, 54, 187

Harwit, M. 1970a, Nature, 226, 61

Harwit, M. 1970b, Bull. Astron. Inst. Czechoslovakia, 21, 204

Hiltner, W. A. 1949a, Science, 109, 165

Hiltner, W. A. 1949b, ApJ, 109, 471

Jones, R. V. \& Spitzer, L. 1967, ApJ, 147, 943

Kim, S.-H. \& Martin, P. G. 1995, ApJ, 444, 293

Landau, L. D. \& Lifshitz, E. M. 1976, Mechanics (Oxford: Pergamon)

Lazarian, A. 2002, astro-ph/0208487

Lazarian, A. \& Draine, B. T. 1997, ApJ, 487, 248

Lazarian, A. \& Draine, B. T. 1999a, ApJ, 516, L37

Lazarian, A. \& Draine, B. T. 1999b, ApJ, 520, L67

Lazarian, A. \& Draine, B. T. 2000, ApJ, 536, L15

Lazarian, A. \& Roberge, W. G. 1997, ApJ, 484, 230

Li, A. \& Draine, B. T. 2001, ApJ, 554, 778

Martin, P. G. 1971, MNRAS, 153, 279

Mathis, J. S. 1986, ApJ, 308, 281

Mathis, J. S., Mezger, P. G., \& Panagia, N. 1983, A\&A, 128, 212

Mezger, P. G., Mathis, J. S., \& Panagia, N. 1982, A\&A, 105, 372

Purcell, E. M. 1975, in The Dusty Universe, ed. G. B. Field \& A. G. W. Cameron (New York: Neal Watson), 155

Purcell, E. M. 1979, ApJ, 231, 404 
Purcell, E.M. \& Spitzer, L. 1971, ApJ, 167, 31

Roberge, W.G. \& Lazarian, L. 1999, MNRAS, 305, 615

Spitzer, L. \& McGlynn, T. A. 1979, ApJ, 231, 417

Spitzer, L. \& Schatzman, E. 1949, AJ, 54, Q194

Spitzer, L. \& Tukey, J.W. 1949, Science, 109, 461

Spitzer, L. \& Tukey, J.W. 1951, ApJ, 114, 187

Wangsness, R. K. 1956, Phys Rev, 104, 857

Weingartner, J. C. \& Draine, B. T. 2001, ApJ, 553, 581

This preprint was prepared with the AAS LATEX macros v5.0.

Table 1. Branching Probabilities

\begin{tabular}{lll}
\hline \hline \multicolumn{1}{c}{ probability } & $f_{0}$ & \multicolumn{1}{c}{ final flip state } \\
\hline$P_{0}$ & 1 & original (no flips) \\
$\left(1-P_{0}\right)\left(1-P_{\mathrm{f}}\right)$ & $f_{s}$ & original (even \# of flips) \\
$\left(1-P_{0}\right) P_{\mathrm{f}}$ & $f_{s}$ & opposite (odd \# of flips) \\
\hline
\end{tabular}



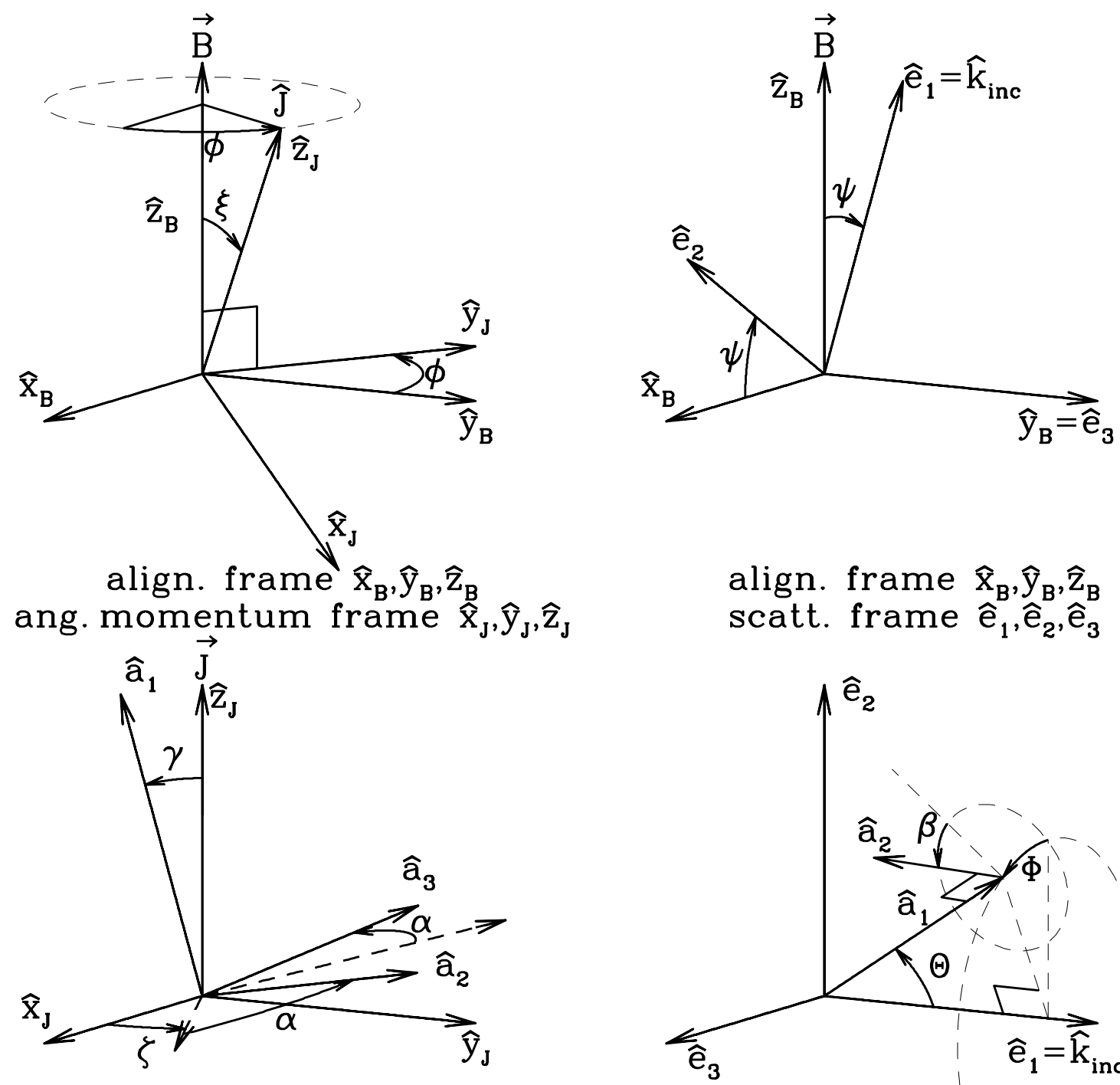

align. frame $\widehat{\mathrm{x}}_{\mathrm{B}}, \widehat{\mathrm{y}}_{\mathrm{B}}, \widehat{\mathrm{z}}_{\mathrm{B}}$ scatt. frame $\widehat{e}_{1}, \widehat{e}_{2}, \widehat{e}_{3}$

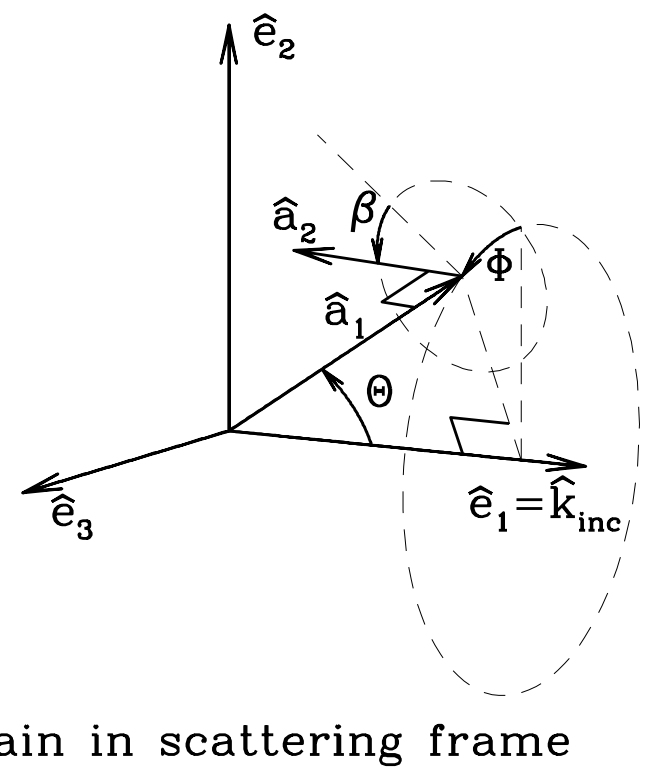

Fig. 1.- Orientation of the "angular momentum" ( $\left.\hat{\mathbf{x}}_{\mathrm{J}}, \hat{\mathbf{y}}_{\mathrm{J}}, \hat{\mathbf{z}}_{\mathrm{J}}\right)$ and "scattering" ( $\left.\hat{\mathbf{e}}_{i}\right)$ coordinate frames in the "alignment" $\left(\hat{\mathbf{x}}_{\mathrm{B}}, \hat{\mathbf{y}}_{\mathrm{B}}, \hat{\mathbf{z}}_{\mathrm{B}}\right)$ coordinate frame and orientation of the grain axes $\hat{\mathbf{a}}_{i}$ in the angular momentum frame and scattering frame. 


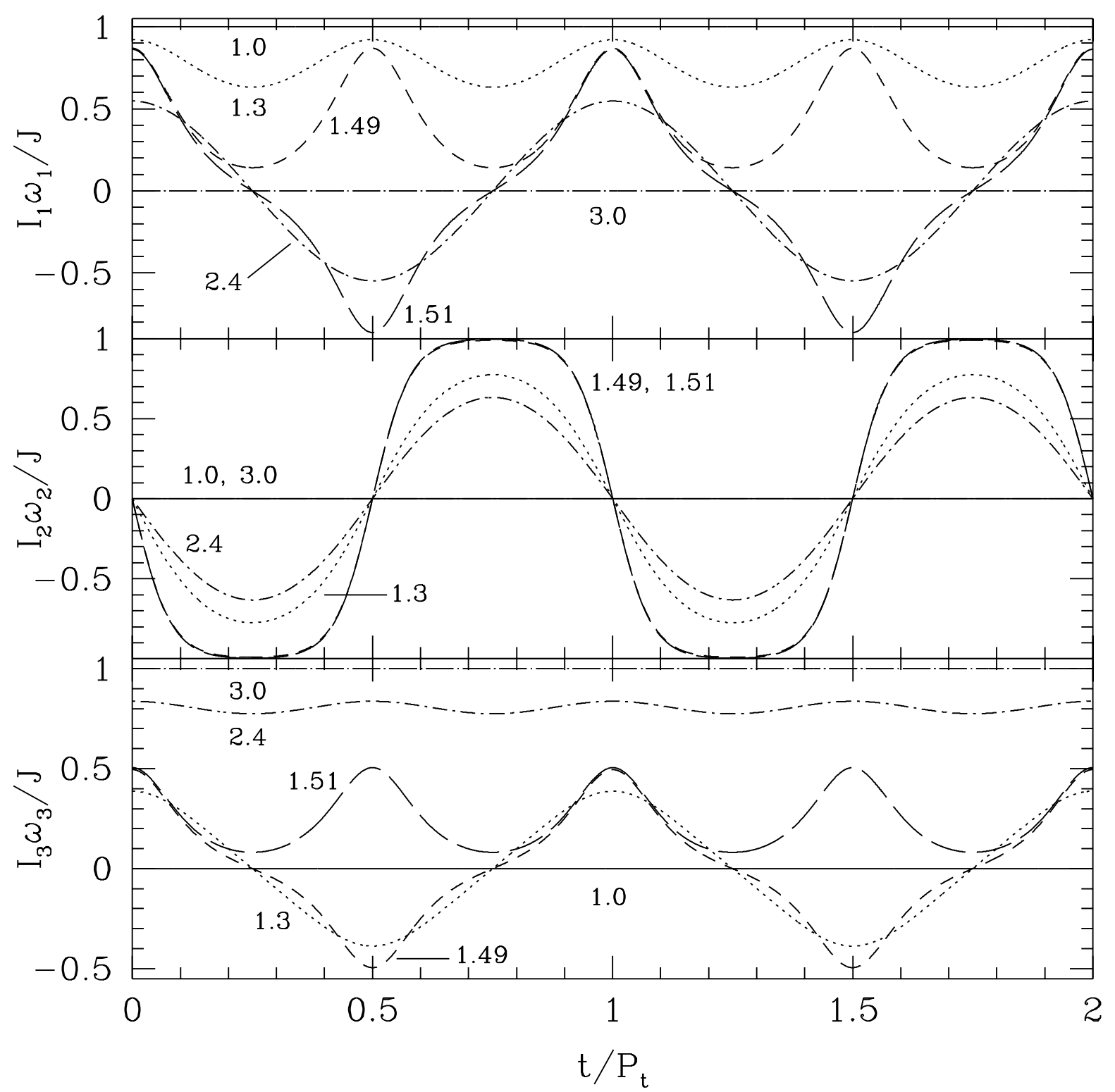

Fig. 2.- Components of the angular velocity along grain principal axes, for $I_{1}: I_{2}: I_{3}=3: 2: 1$. Values of $q$ are indicated and positive flip states are assumed. 


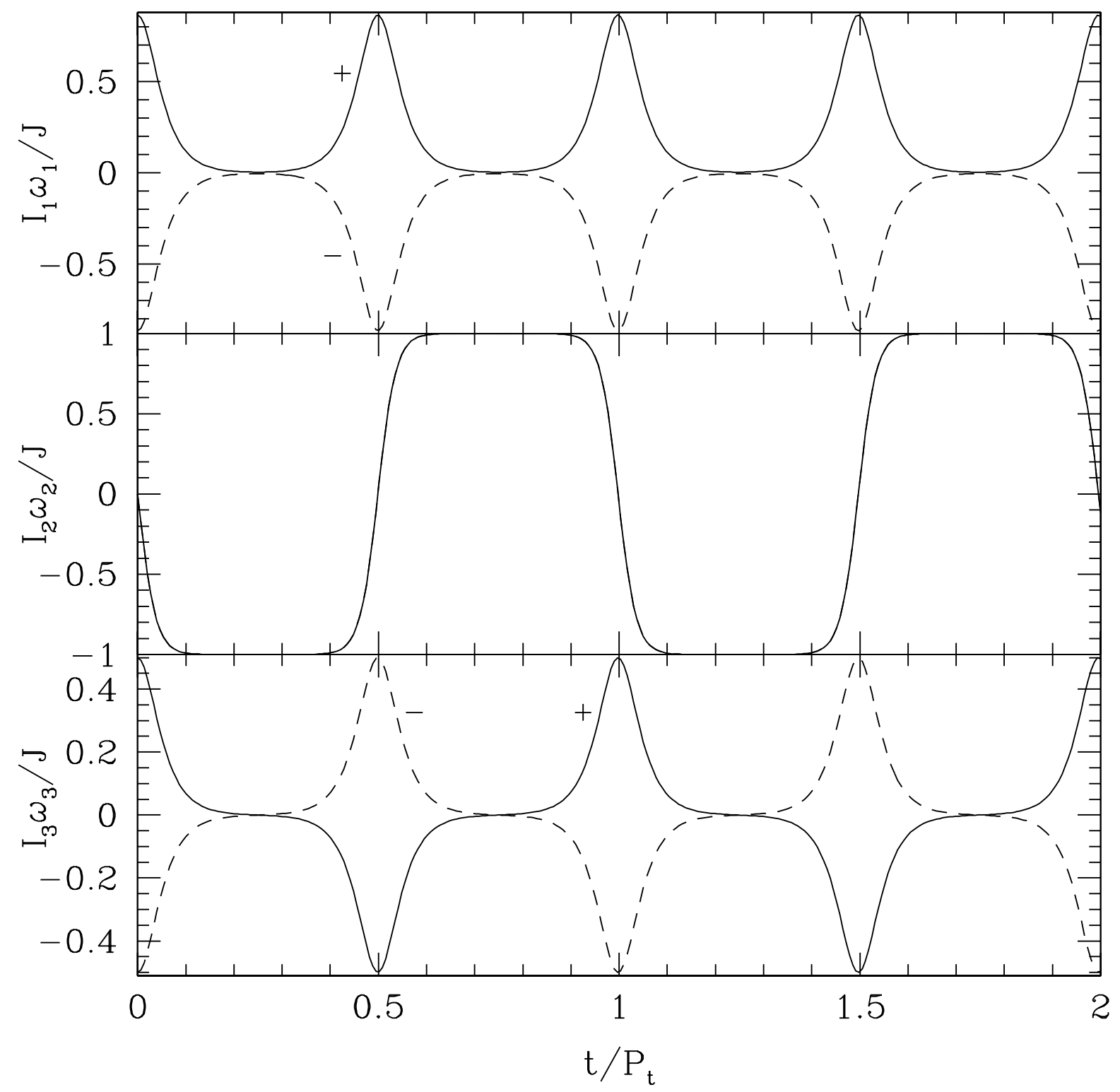

Fig. 3.- Components of the angular velocity along grain principal axes, for $I_{1}: I_{2}: I_{3}=3: 2: 1$ and $q$ slightly less than $I_{1} / I_{2} ;+(-)$ indicates the positive (negative) flip state with respect to $\hat{\mathbf{a}}_{1}$. $\omega_{2}$ does not depend on the flip state. 


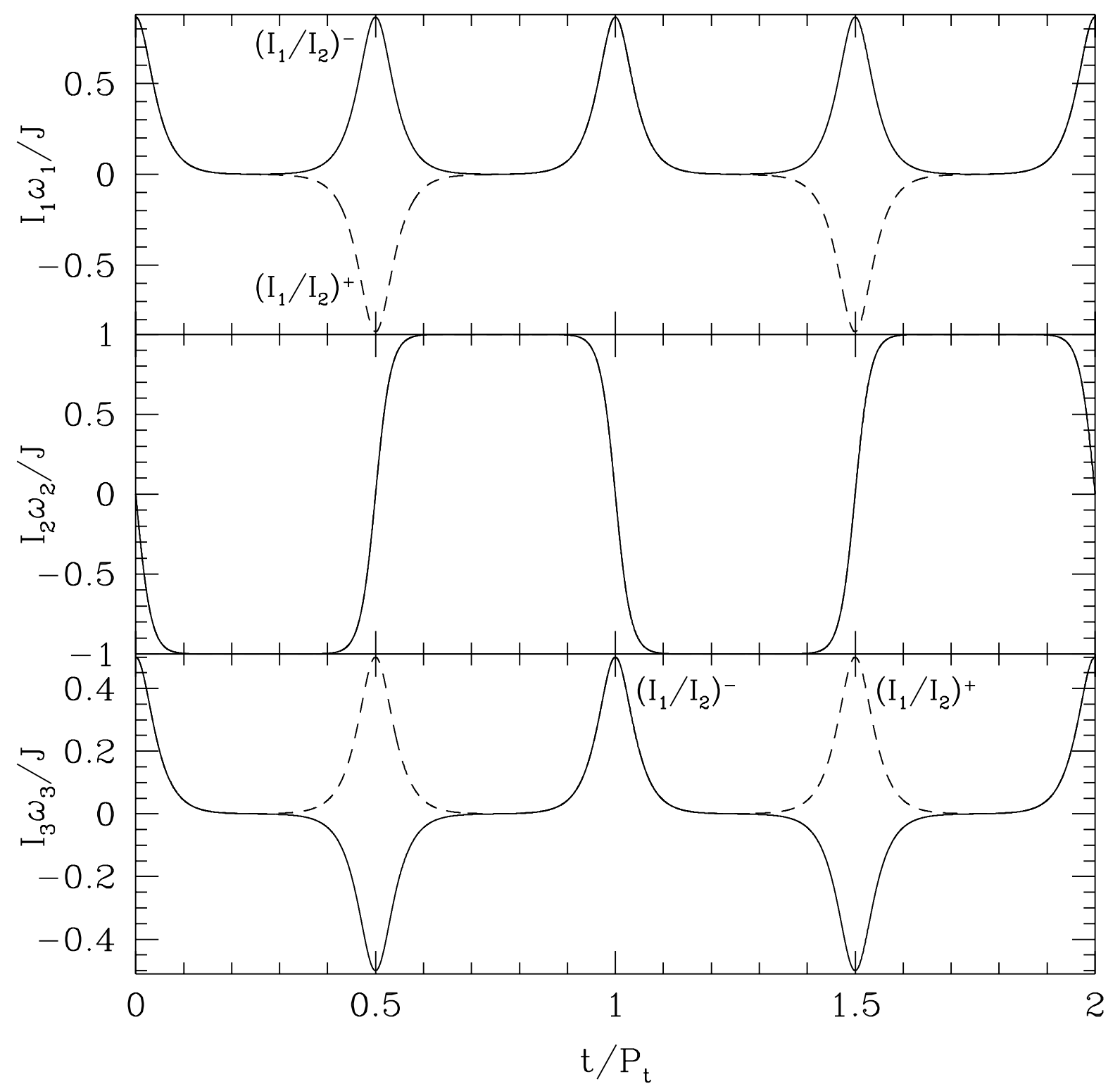

Fig. 4. - Components of the angular velocity along grain principal axes, for $I_{1}: I_{2}: I_{3}=3: 2: 1$. $\left(I_{1} / I_{2}\right)^{+}$and $\left(I_{1} / I_{2}\right)^{-}$indicate that $q$ is slightly larger or smaller than $I_{1} / I_{2}$, respectively. The curves shown are for the positive flip states with respect to $\hat{\mathbf{a}}_{1}$ and $\hat{\mathbf{a}}_{3}$. The two curves are equivalent in regions where the dashed curve is absent. 


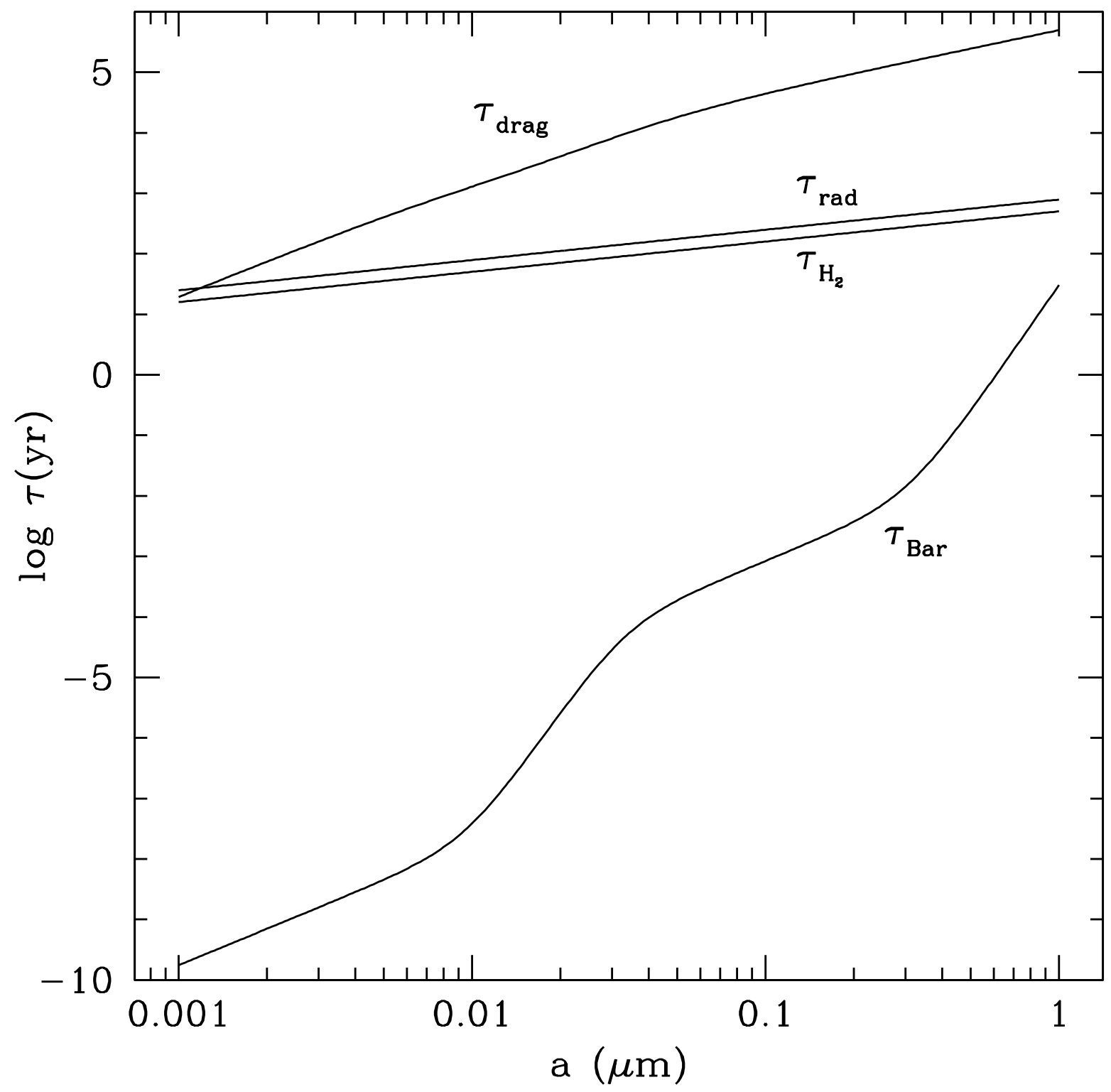

Fig. 5. - Timescales for Barnett relaxation $\left(\tau_{\text {Bar }}\right)$, the drag torque $\left(\tau_{\text {drag }}\right)$, the $\mathrm{H}_{2}$ formation torque $\left(\tau_{\mathrm{H}_{2}}\right)$, and radiative torques $\left(\tau_{\mathrm{rad}}\right)$ for silicate grains, assuming $\omega=\omega_{\mathrm{T}}, I_{1} \approx 2 I_{3}, T_{d}=15 \mathrm{~K}$, $\rho=3 \mathrm{~g} \mathrm{~cm}^{-3}, T=100 \mathrm{~K}, n_{\mathrm{H}}=30 \mathrm{~cm}^{-3}, u_{\mathrm{rad}}=u_{\mathrm{ISRF}}, f=1, l=10 \AA, E_{\mathrm{H}_{2}}=0.2 \mathrm{eV}, n(\mathrm{H})=n_{\mathrm{H}}$, and $\gamma_{\text {rad }} H=10^{-3}$. Both $\tau_{\mathrm{H}_{2}}$ and $\tau_{\text {rad }} \propto a^{0.5}$ and, coincidentally, are nearly identical for the above canonical conditions. 


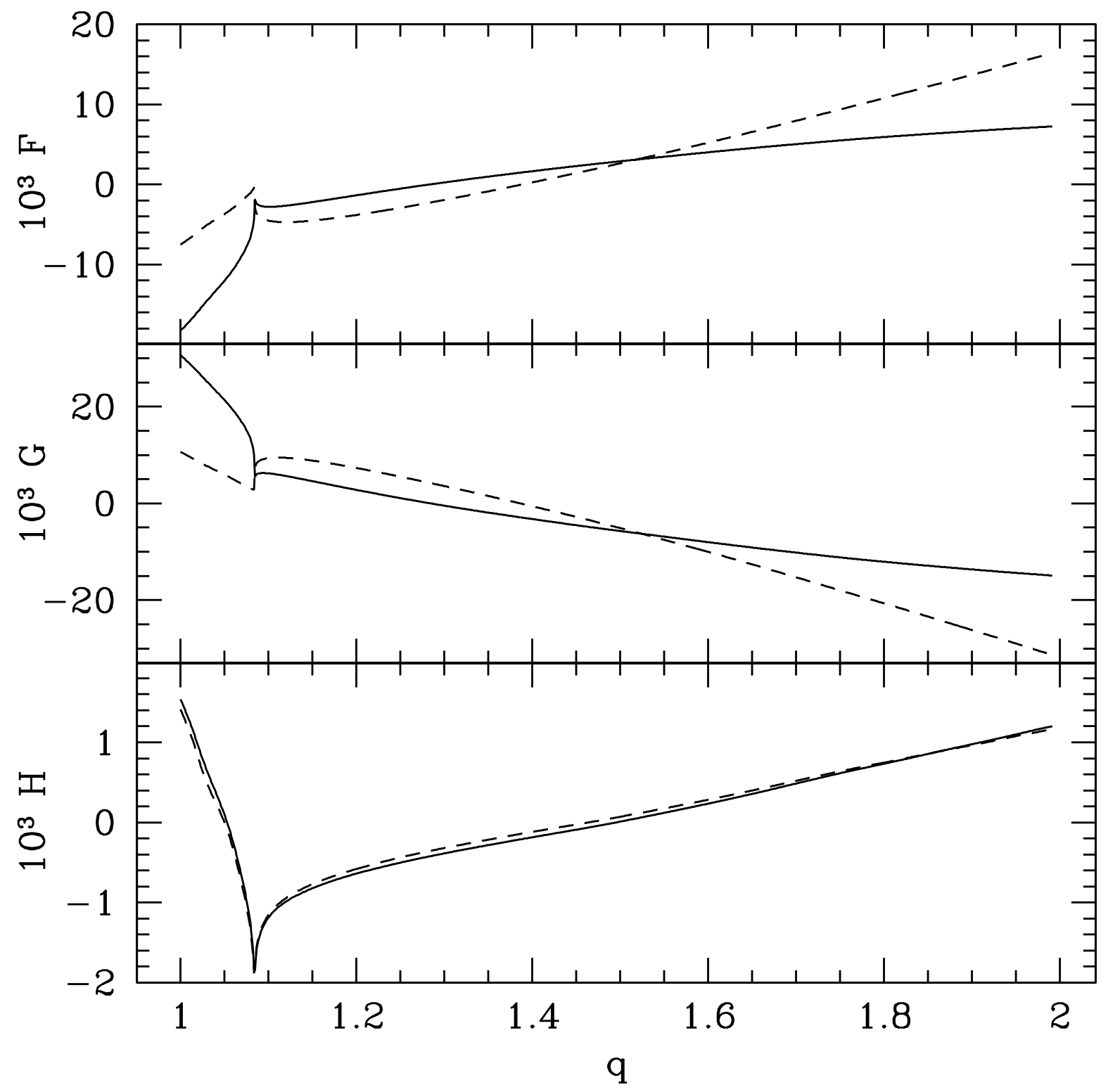

Fig. 6. $-F, G$, and $H$ for shape 1 , astronomical silicate, $a=0.2 \mu \mathrm{m}, \psi=70^{\circ}, \xi=30^{\circ}, \phi=160^{\circ}$, and positive (solid) and negative (dashed) flip states. A monochromatic radiation field with $\lambda=$ $\tilde{\lambda}_{\mathrm{ISRF}}=1.2 \mu \mathrm{m}$ was adopted. 


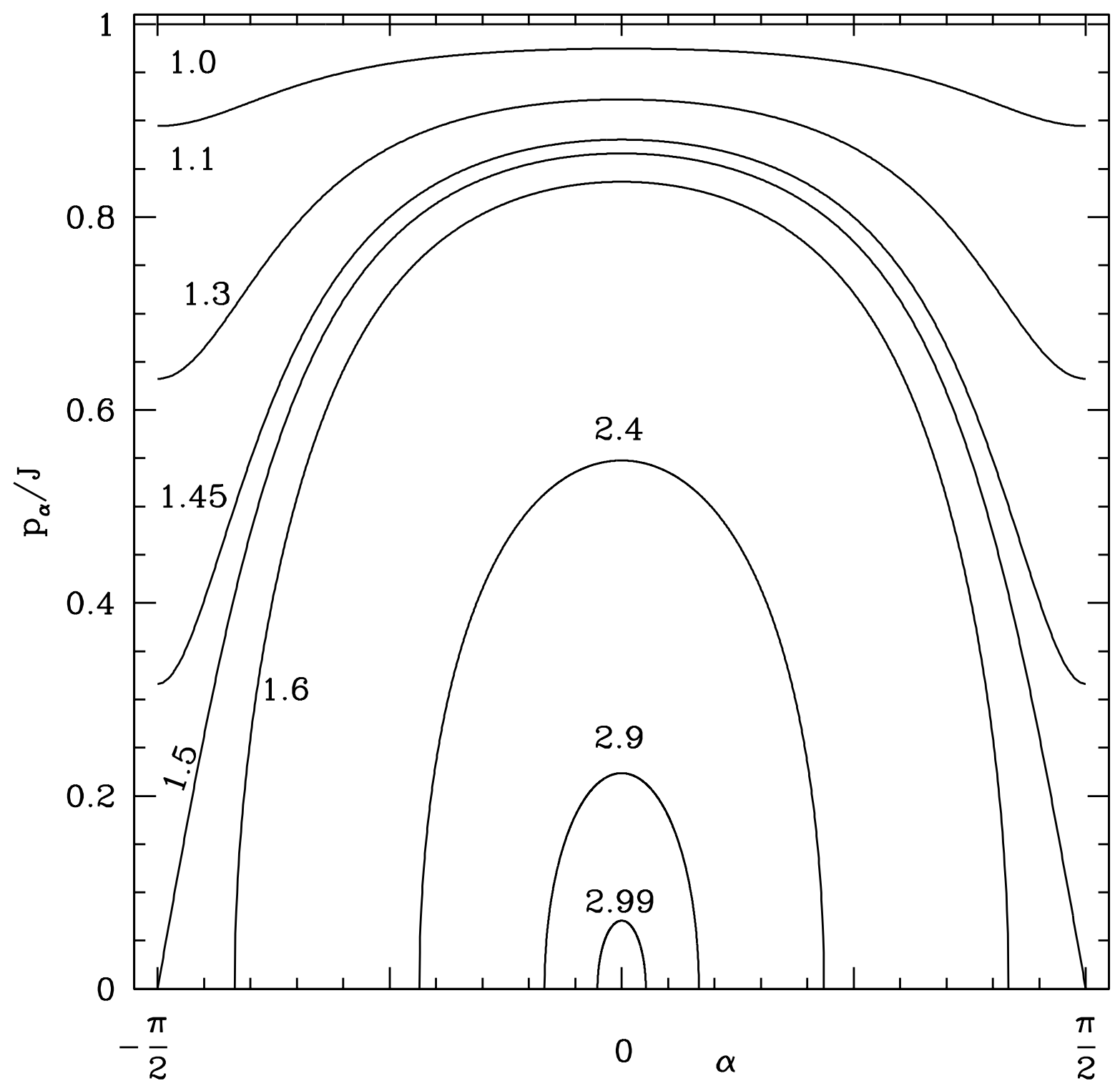

Fig. 7.- Phase space trajectories for the case that $I_{1}: I_{2}: I_{3}=3: 2: 1$. The Eulerian angle $\alpha$ was introduced in $\S 2.5 .3$ and $p_{\alpha}$ is its conjugate momentum. The values of $q$ are indicated. 


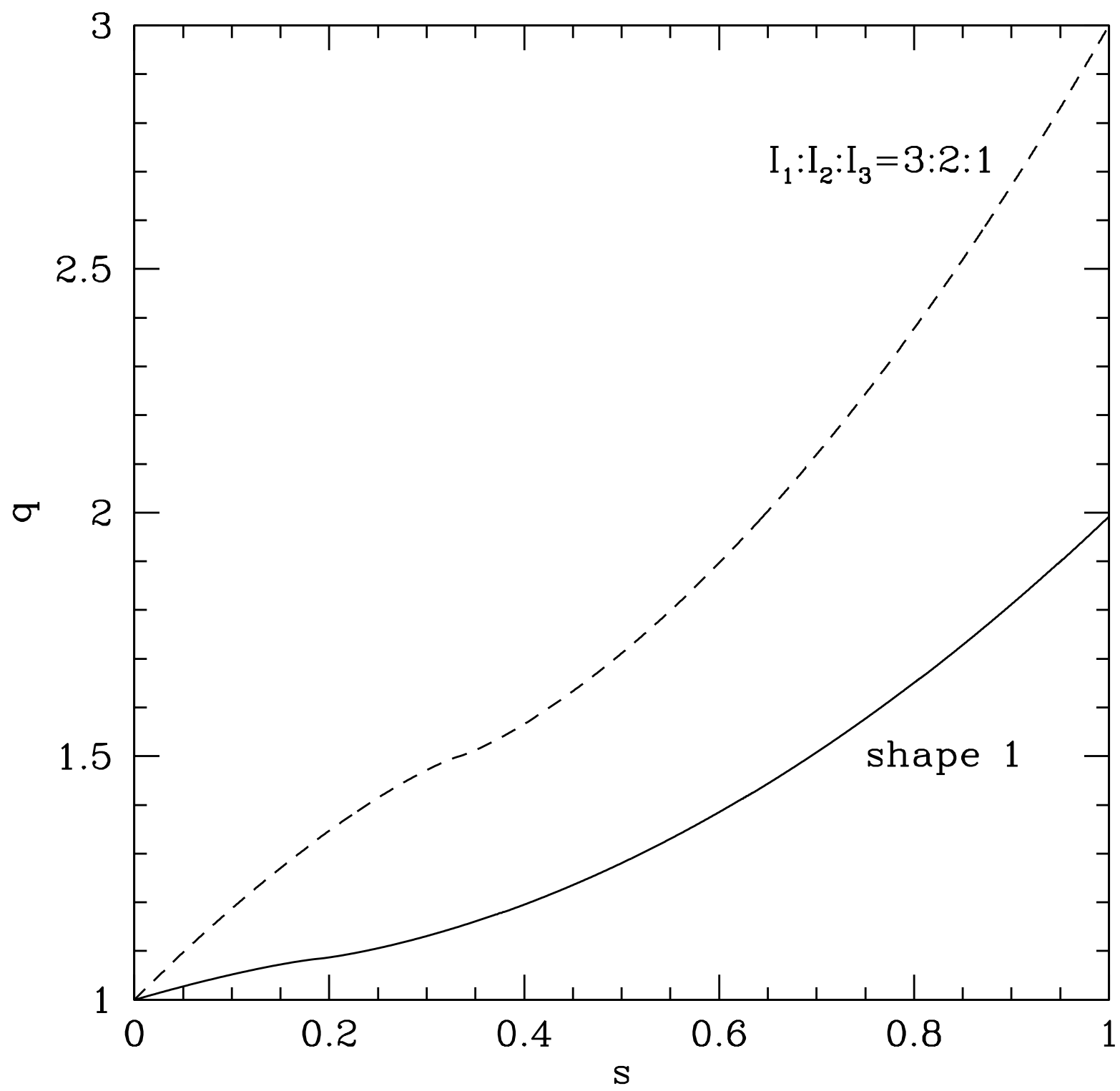

Fig. 8. $-q$ versus $s$ for a grain with $I_{1}: I_{2}: I_{3}=3: 2: 1$ and for shape 1 from Paper I. 


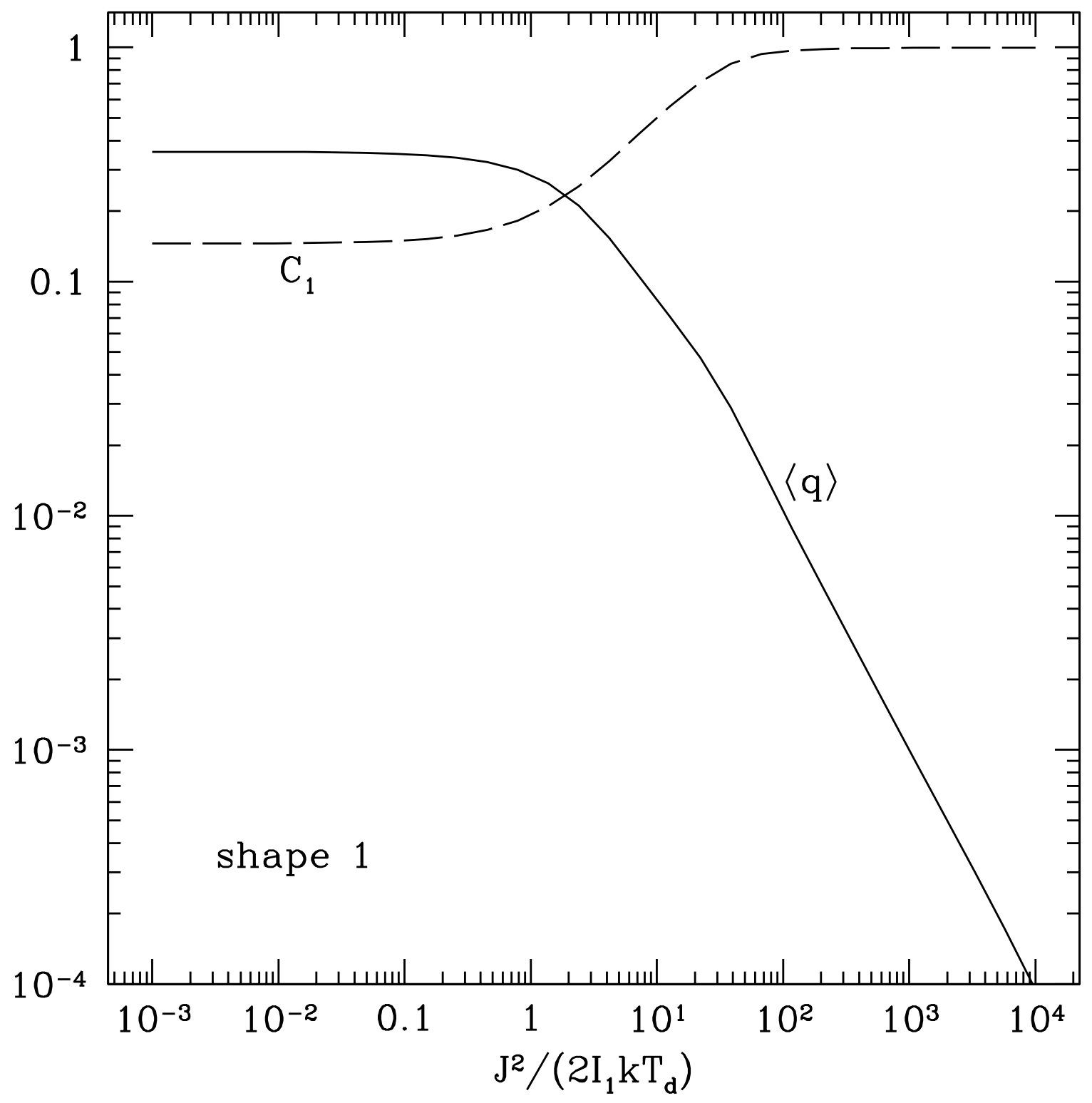

Fig. 9.- $\langle q\rangle$ and $C_{1}$. 


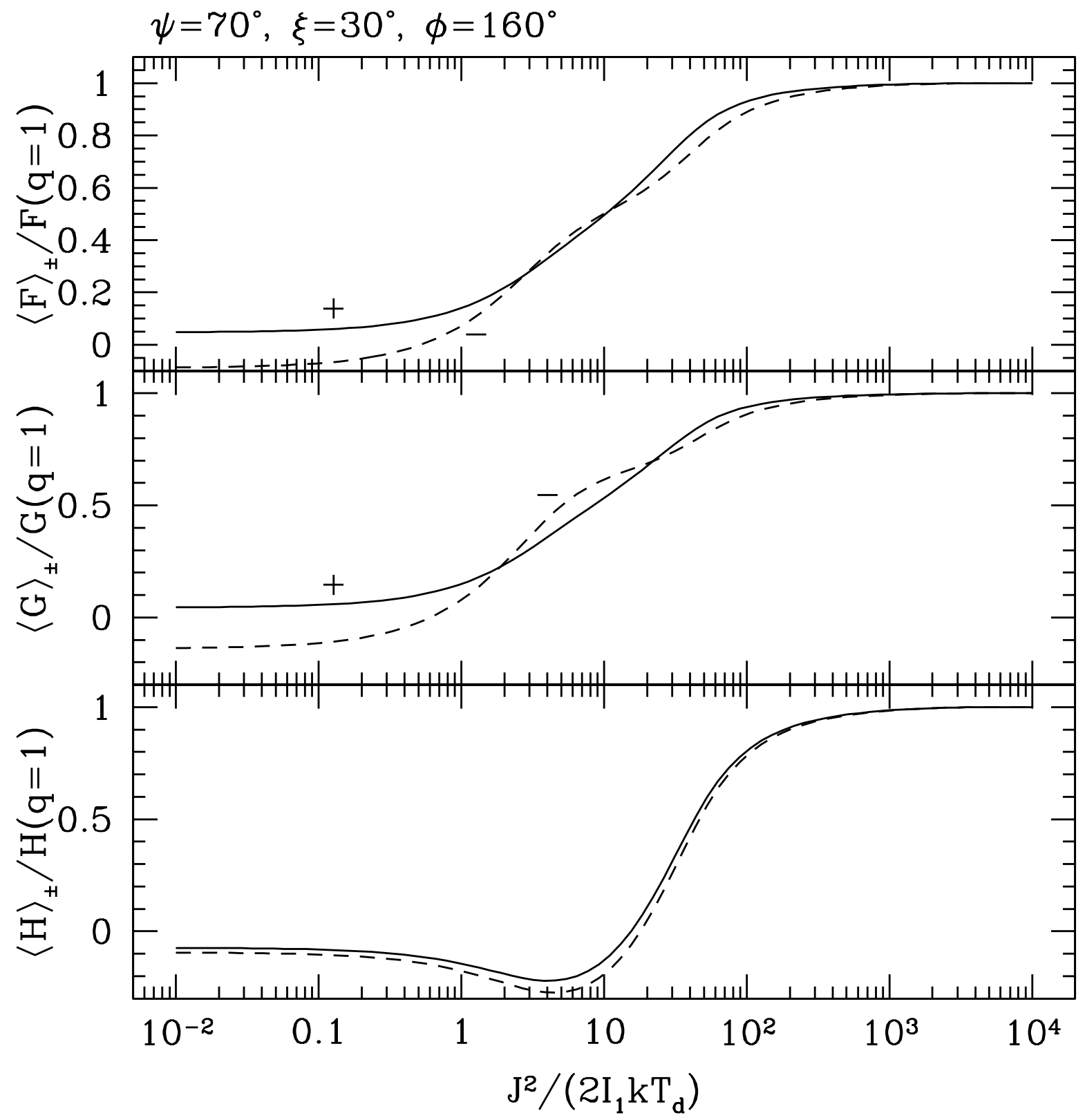

Fig. 10. $-\langle F\rangle_{ \pm},\langle G\rangle_{ \pm}$, and $\langle H\rangle_{ \pm}$, normalized to their values when $q=1$, for shape 1, one particular angular momentum direction $(\xi, \phi)$ and starlight anisotropy direction $\psi$. 


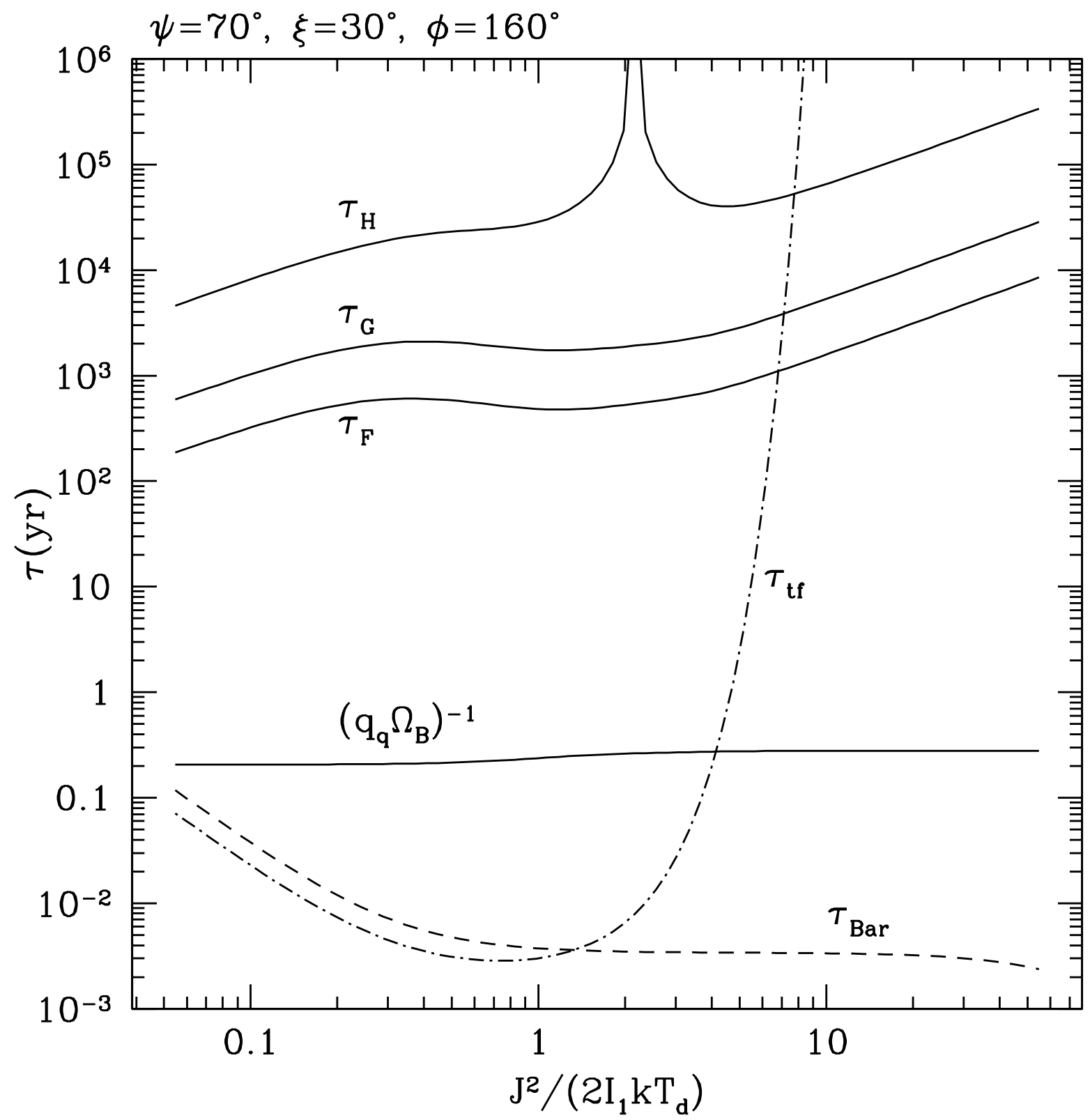

Fig. 11. - The Barnett dissipation timescale $\tau_{\text {Bar }}$, the thermal flipping timescale $\tau_{\text {tf }}$, and timescales associated with various terms in the equations of motion: $\tau_{F} \equiv 2 J /\left(\gamma_{\mathrm{rad}} u_{\mathrm{rad}} a^{2} \tilde{\lambda}\langle F\rangle_{+}\right), \tau_{G} \equiv$ $2 J \sin \xi /\left(\gamma_{\mathrm{rad}} u_{\mathrm{rad}} a^{2} \tilde{\lambda}\langle G\rangle_{+}\right)$, and $\tau_{H} \equiv 2 J /\left(\gamma_{\mathrm{rad}} u_{\mathrm{rad}} a^{2} \tilde{\lambda}\langle H\rangle_{+}\right)$, for shape $1, a=0.2 \mu \mathrm{m}, T_{d}=15 \mathrm{~K}$, $\psi=70^{\circ}, \xi=30^{\circ}$, and $\phi=160^{\circ}$. 


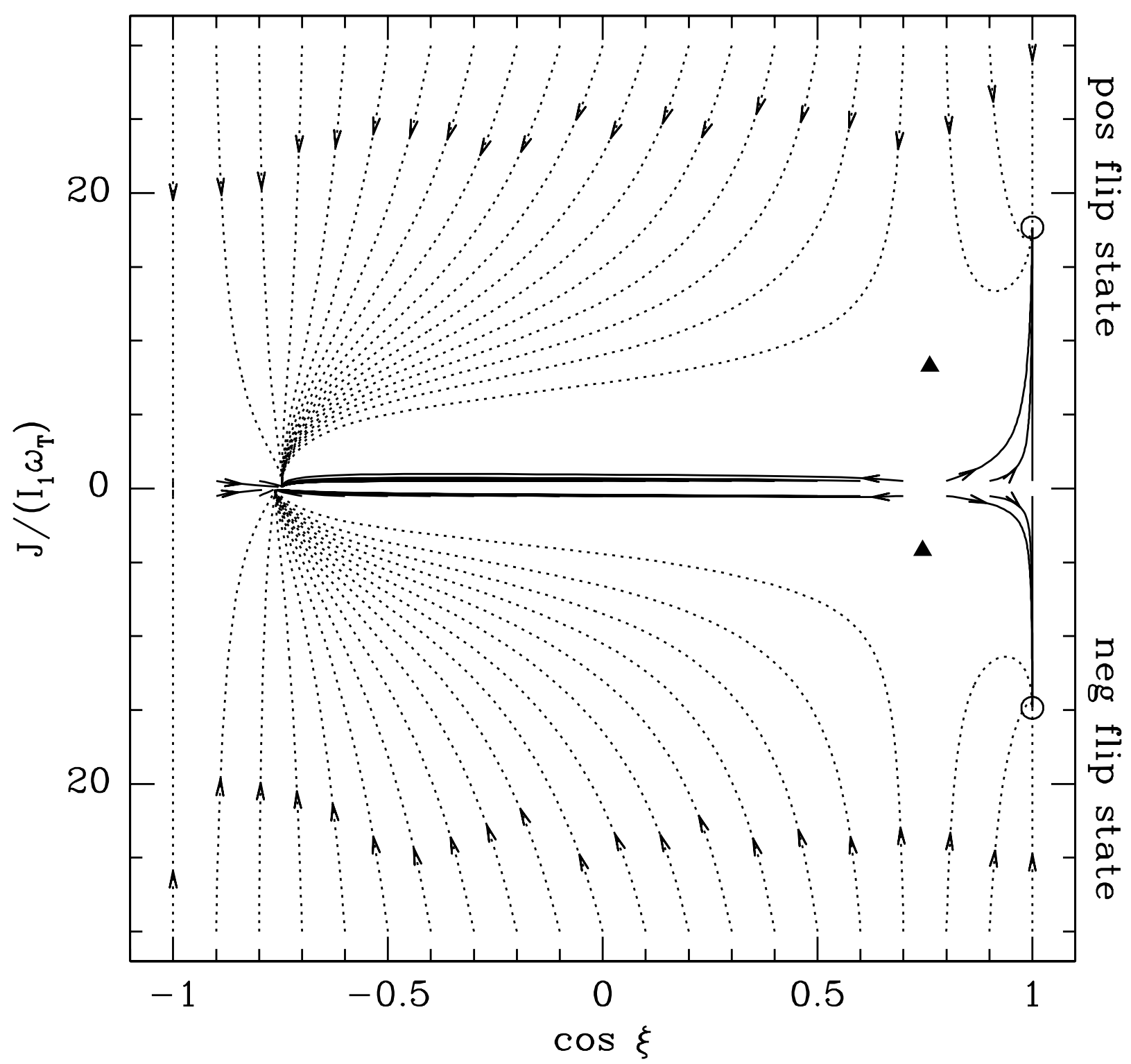

Fig. 12.- Trajectories computed using the method of Paper II (i.e., constraining $\mathbf{J}$ to be parallel or anti-parallel to $\hat{\mathbf{a}}_{1}$ ) for $\psi=70^{\circ}$ (see text for specification of other parameter values). Dotted (solid) curves originate at $J / I_{1} \omega_{\mathrm{T}}=30(0.5)$. Attractors (repellors) are indicated by open circles (filled triangles). 


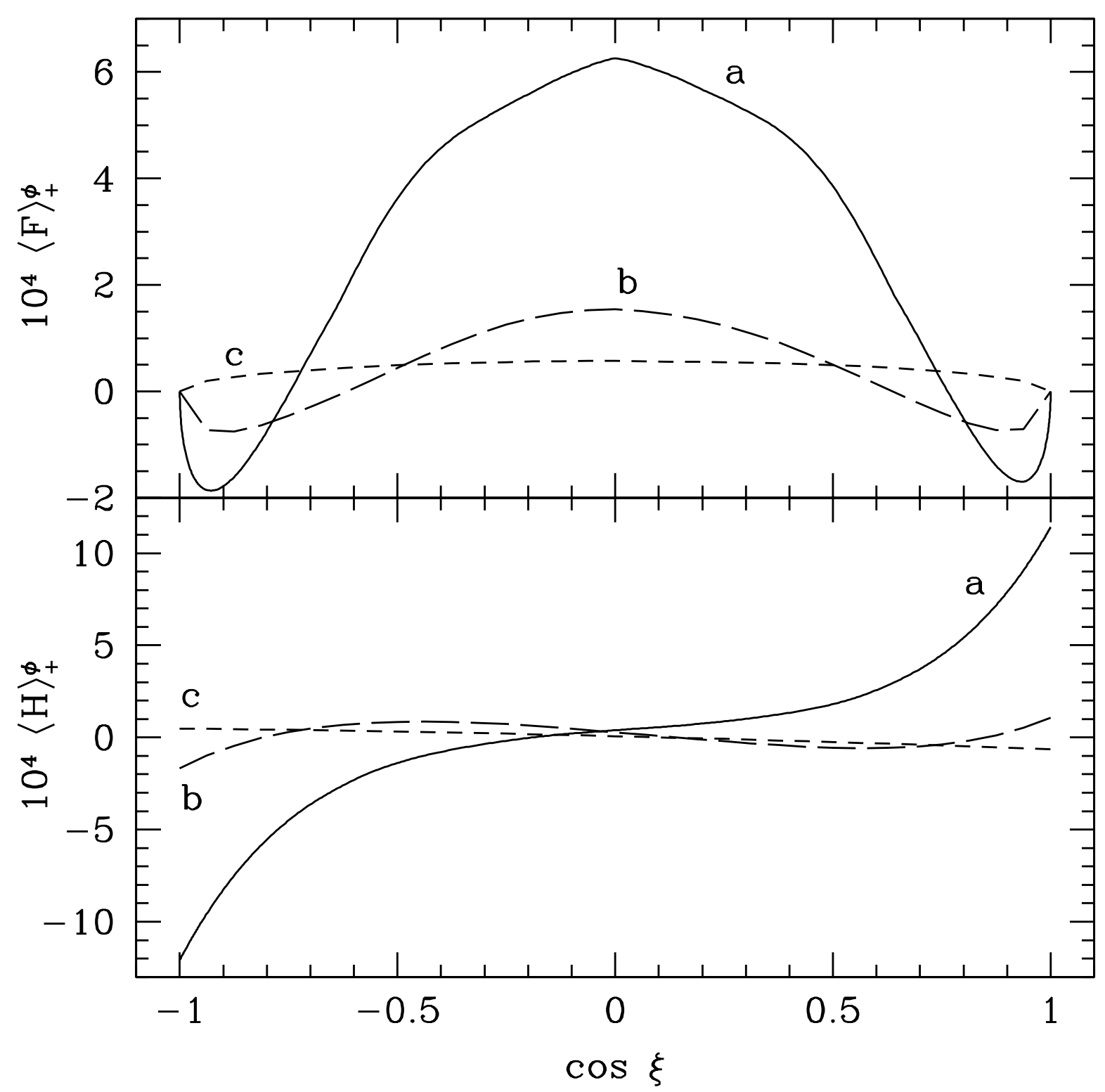

Fig. 13. $-\langle F\rangle_{+}^{\phi}$ and $\langle H\rangle_{+}^{\phi}$ versus $\cos \xi$ for shape 1 and $\psi=70^{\circ}$. a) $J \rightarrow \infty ; \mathrm{b}$ ) $J / I_{1} \omega_{\mathrm{T}}=1.7$ (for $T_{\text {gas }}=100 \mathrm{~K}$ and $\left.T_{d}=15 \mathrm{~K}\right)$; c) $J \rightarrow 0$. 


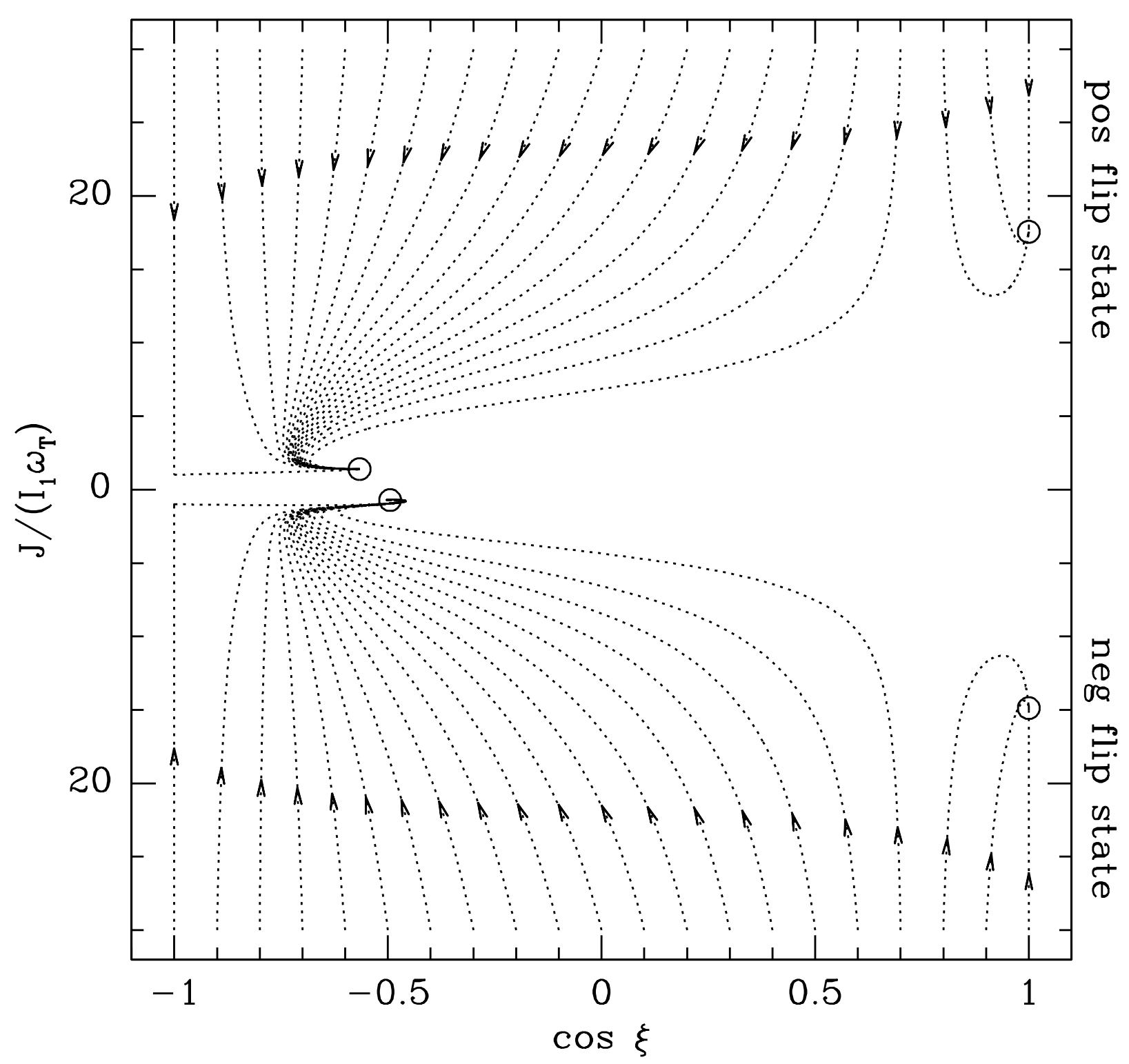

Fig. 14. - Trajectory map (with same parameters as for Figure 12) computed using the method developed in this paper (i.e., relaxing the constraint that $\mathbf{J}$ must be parallel or anti-parallel to $\hat{\mathbf{a}}_{1}$ ), except that thermal flipping is prohibited (see text for details). 


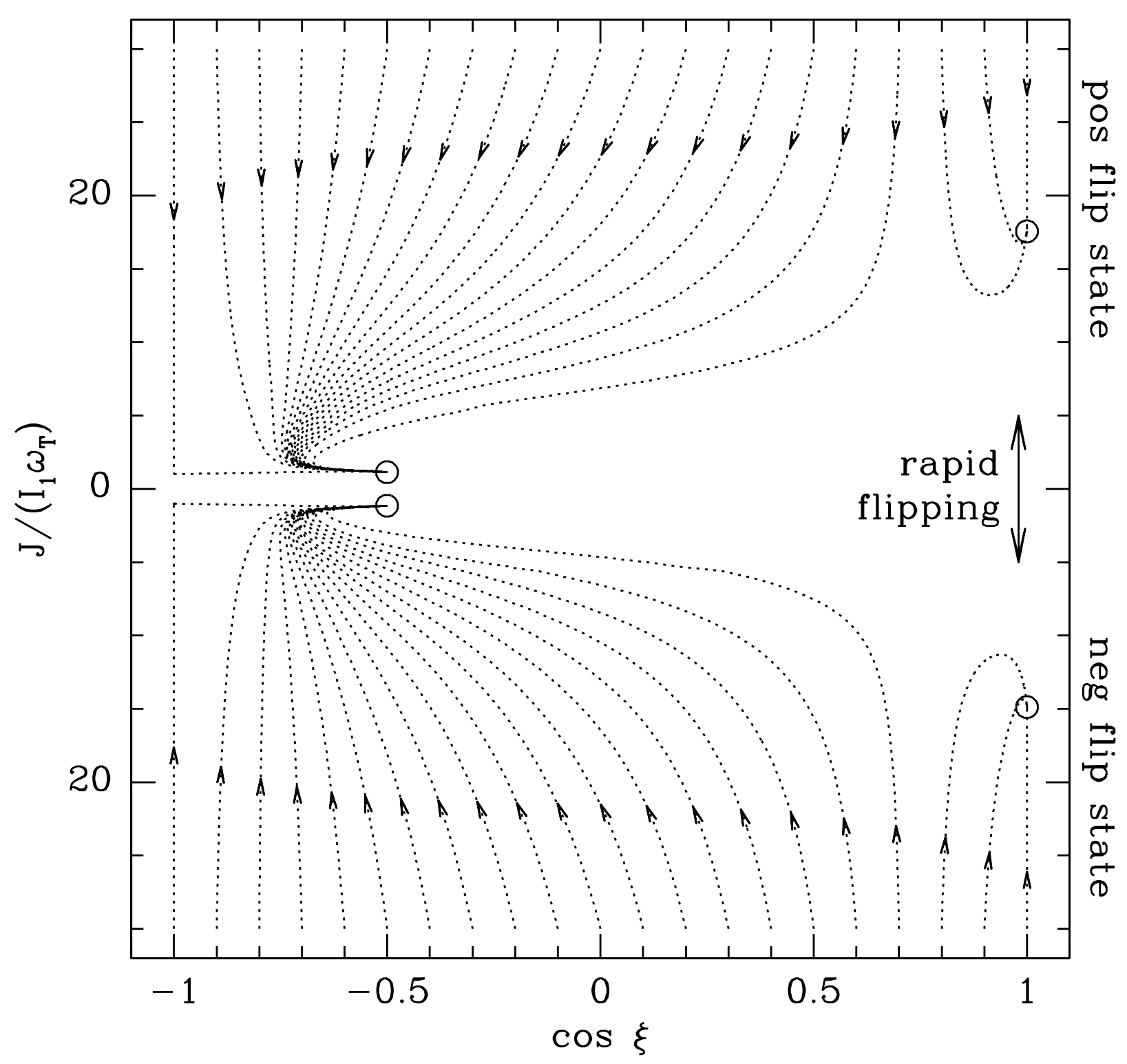

Fig. 15.- Same as Fig. 14, but with thermal flips partially included (see text for details). The grain undergoes rapid flipping when $J / I_{1} \omega_{\mathrm{T}} \lesssim 5$. 


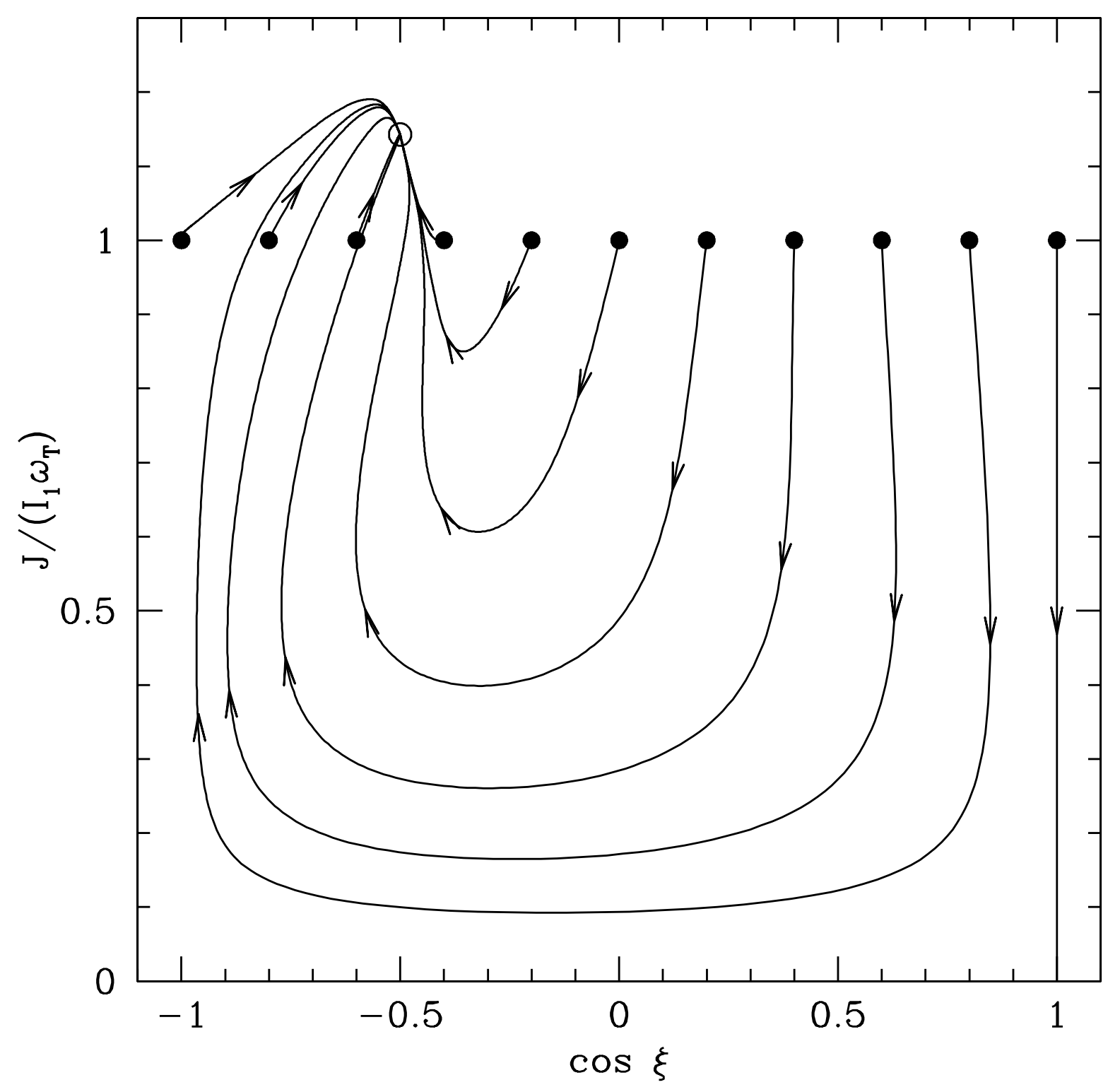

Fig. 16. - Same as Figure 15, but for trajectories starting with $J / I_{1} \omega_{\mathrm{T}}=1$ (initial points are indicated by filled circles). The attractor, on which most trajectories end, is indicated by an open circle. The grain undergoes rapid flipping for all points in this figure. 